Historic, archived document

Do not assume content reflects current scientific knowledge, policies, or practices. 

THE

\section{UNITED STATES \\ NURSERIES}

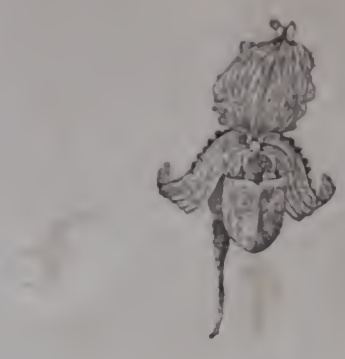

1892

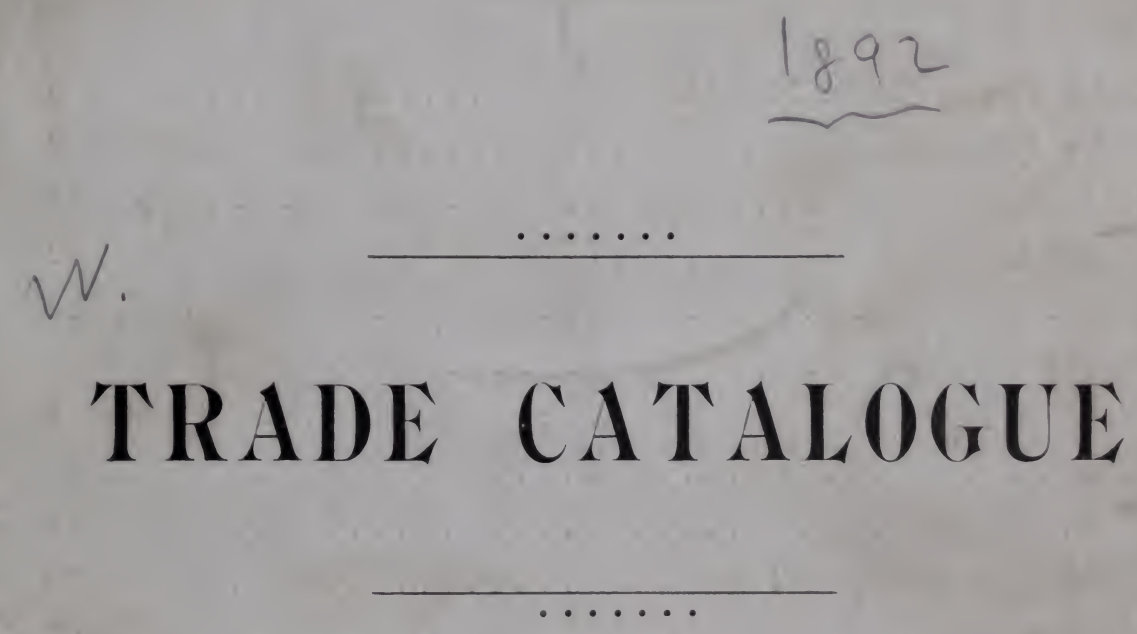

PITCHER \& MANDA,

EUROPEAN BRANCH :

Short Hills,

HEXTABLE, KENT.

NEW JERSEY.

ENGLAND.

SPRING, 189:. 


\section{CONTENTS.}

Choice Flower Seeds, Novelties................... 5

Choice Flower Seeds, in Bulk. . . . . . . . . . . . . . . . 9

Hardy Perennial Seed . . . . . . . . . . . . . . . . 18

General List of Bulbs . . . . . . . . . . . . . . . . 22

Vegetable Seeds . . . . . . . . . . . . . . . . . . . . . 29

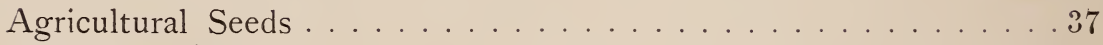

Chrysanthemums . . . . . . . . . . . . . . . . . . . . t1

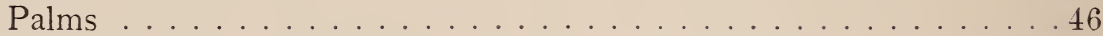

Ferns . . . . . . . . . . . . . . . . . . . . . . . .49

Flowering and Foliage Plants ............. . . . 0

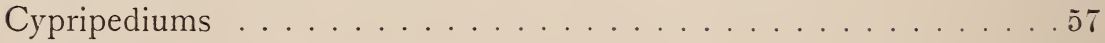

Hardy Perennials or Herbaceous Plants ............ . . 8

Clematis . . . . . . . . . . . . . . . . . . . . . . . . 69

Special Imported Stock for Florists . . . . . . . . . . . . 69

Special Offers to Florists for Easter and Store Decorations. . . . . . 70

Implement and Garden Requisites . . . . . . . . . . . 73

Horticultural Books . . . . . . . . . . . . . . . . . .77 


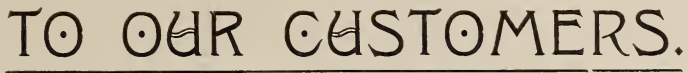 \\ -}

The United States Nurseries are situated in Short Hills, N. J., on the D., L. \& Western R. R., only 17 miles from New York City. We are pleased to see visitors at any time. A personal visit will facilitate selection of plants and afford an opportunity of seeing the extent of our nurseries.

Terms : Accounts are made up monthly. Our patrons are requested to remit by checks, express, or P. O. money orders on New York Post Office, or currency in registered letters, as we cannot be responsible for money in unregistered letters. From unknown correspondents either a remittance or a reference is required with each order.

No Charges are made for boxes, packing or delivery to the railroad or express office; bags only are charged for. All plants are carefully packed and may be forwarded safely to any part of the globe.

Orders are Solicited at as early a date as possible, that we may have ample time for their execution. Please write plainly, name, post office, express office, and any other necessary information as to the best method of forwarding.

Advices of Shipment, together with the invoice, are sent the same day goods are forwarded.

Complaints, if any, should be made on receipt of goods. We promise prompt and just treatment of all claims presented. We guarantee safe arrival of plants sent by express, but do not assume any responsibility if sent by freight.

Our American seeds have been grown for us by men who are the acknowledged leaders in this branch of farming in the country, and our imported seeds have been procured from the most reliable sources abroad. The crops of many of the leading kınds of vegetable seeds are short this year. We have taken every pains to have the original stock pure and true ; the crops have been personally inspected while growing by our representative ; and every care has been taken that the seed be properly ripened, carefully dried and cleaned, and all inferior and imperfect specimens rejected.

Prices are subject to change without notice, and to the goods being unsold on receipt of order. We would advise dealers to place their orders early, as prices are likely to advance at the beginning of the selling season.

Our travelers are in all sections of the country the year round and would be pleased to call upon notification to that effect, with prices and samples.

We import, grow and deal extensively in the following, and are prepared, with the heavy stock we carry, to quote advantageous prices:

Choice Flower Seeds, Reliable Vegetable Seeds, Rare Orchids, New Chrysanthemums, Scarce and Popular Palars, Magnificent Foliage Plants, Ferns of every Description and Useful Hardy Plants. 


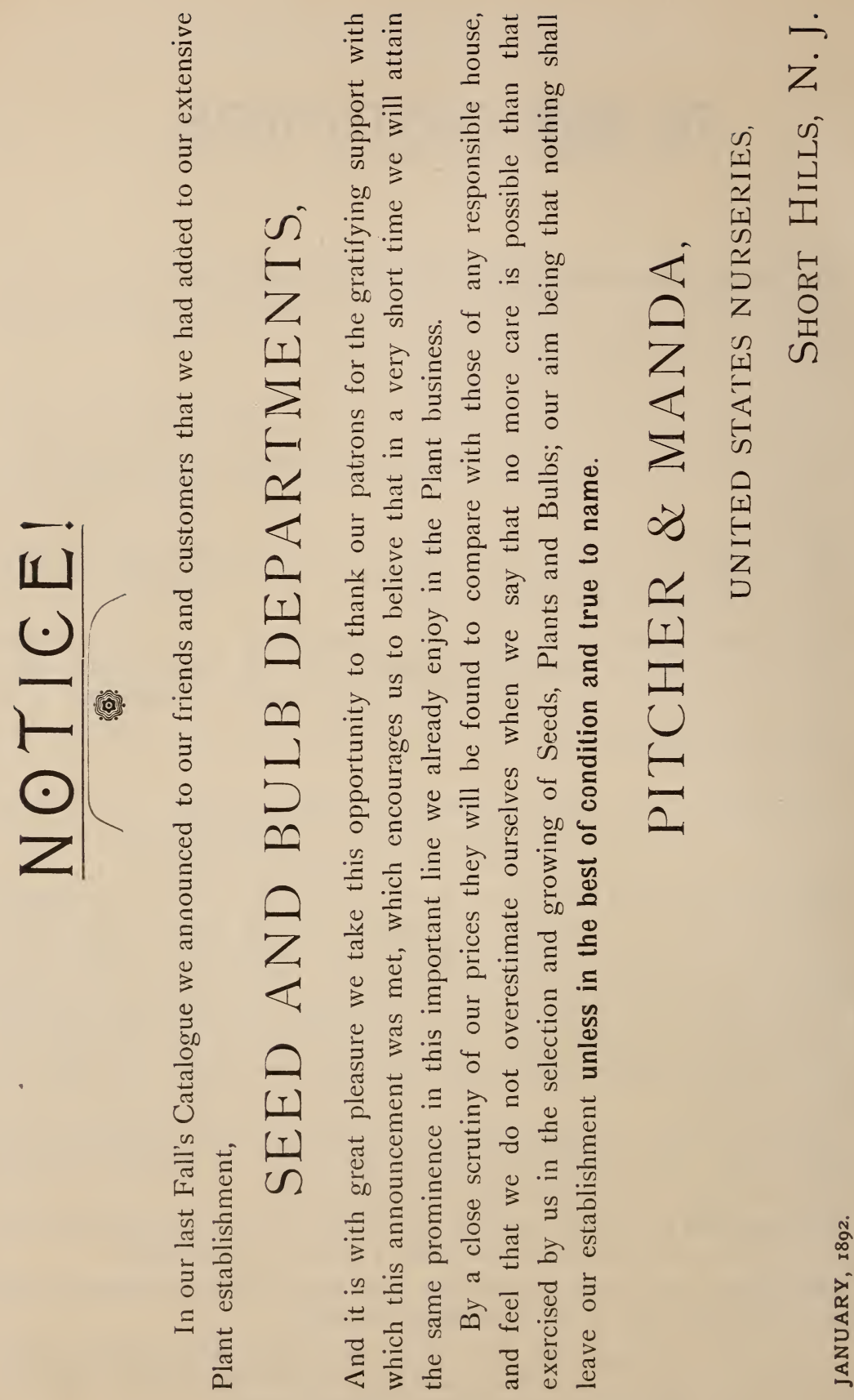




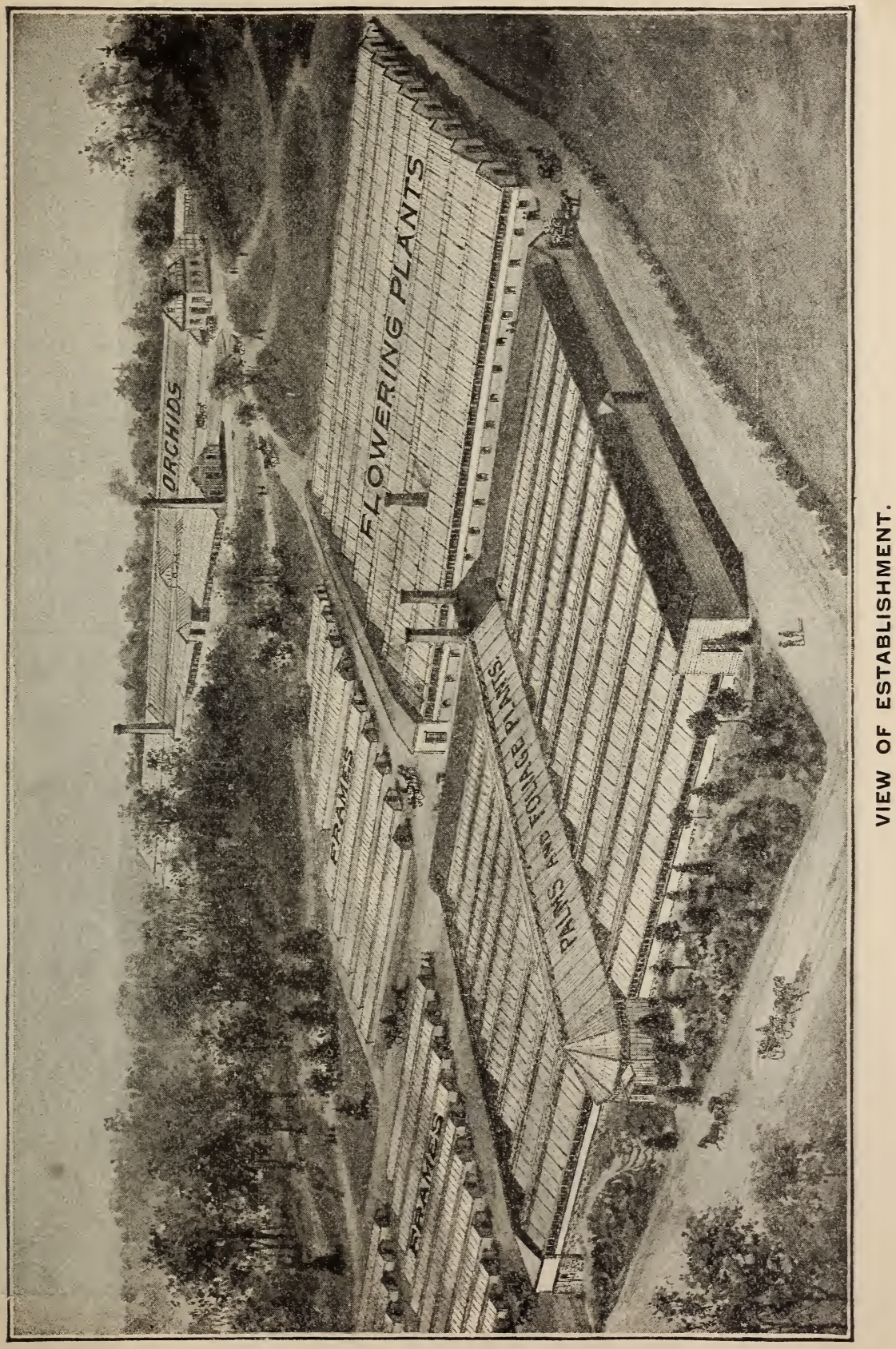




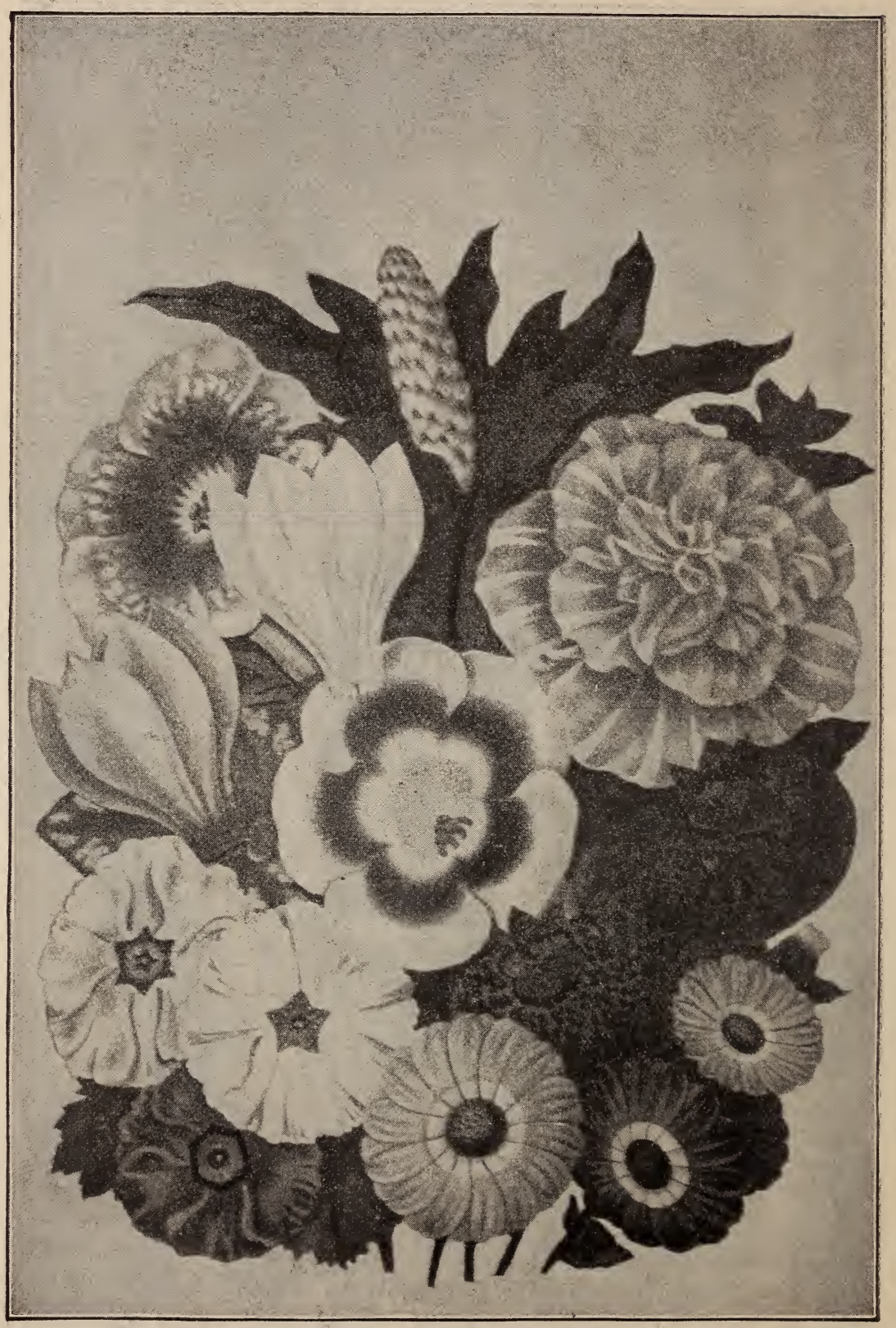

Choice Strains of Floristis' Fiowers. 


\section{Choice Flower Seed Novelties.}

\section{TUBEROUS BEGONIAS.}

This is a class of plants which has been developed within the past $I_{5}$ or 20 years by the skill of the hybridizer. Now we have in them bright scarlet, more brilliant than Geraniums, and in all shades of rose-red, pink to white, and of late we have brilliant yellows, from pale strawcolor to brilliant bronze, and all both double and single. Our collection of these plants is very large, and the past season we have carefully saved seed from the best flowers, which we offer as below. This seed of our own saving is, we assure our customers, superior to the imported seed usually offered.

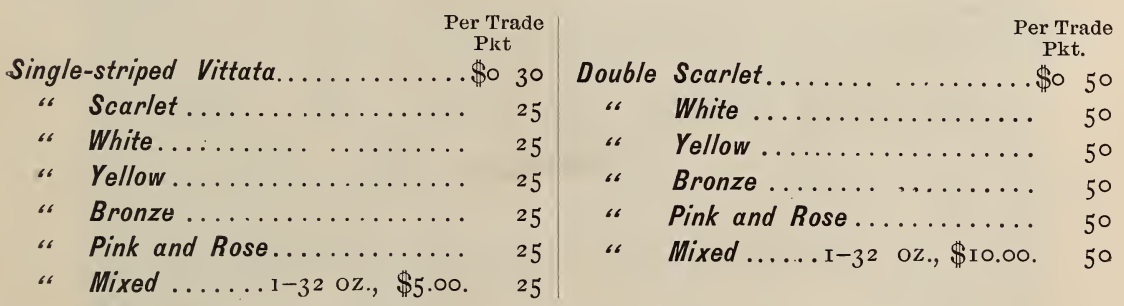

\section{OUR PRIZE CHRYSANTHEMUM SEED.}

We are now universally considered headquarters for this grand flower, our collection taking the 'best prizes wherever exhibited. Instead of selling our cut-flowers, we take special pains to induce the choicest varieties to seed by artificial and other means, and have met with such success that we will have for sale a large quantity, including all types and colors, I5c. per packet, \$I.50 per dozen.

\section{CYPRIPEDIUM.}

The many calls and inquiries for Orchid seed induced us to save and offer this year in our Spring Catalogue, artificially Hybridized Cypripedium Seed.

We have been successful in raising many new and very valuable varieties from seed ourselves, and believe, from the increasing interest taken in this class of plants, that the seed will meet with a ready sale. $30 \mathrm{c}$. per packet, $\$ 3.00$ per dozen. On each packet will appear the Cross of Hybridization,

\section{THE COMET WHITE ASTER.}

This is a lovely new Aster, being sent out for the first time. It is a French novelty and the flowers resemble a Japanese Chrysanthemum; the petals are long, pure-white, and arranged in :a way entirely distinct from most asters. Per pkt., 25 cts.

\section{NEW ROSE COLOR AND WHITE “BALL" ASTER.}

This novelty bears very double flowers, with very symmetrically incurving petals, making the flower almost exactly spherical. They are rose color and white, and very distinct among Asters, deserving general cultivation. Per pkt., 15 cts. 


\section{CARDINAL POPPY.}

This is a variety of Papaver somniferum and is distinguished from the type by its dwarfer habit of growth and the size and brilliant color of its flowers. These are immense, very double, brilliant scarlet on a white ground, and are borne Io to I2 on a plant I8 inches high, lasting for a long time in perfection. A grand annual Poppy which we predict will prove a decided acquisition. Per pkt., I5 cts.

\section{NEW GLOXINIA "CORONA."}

This grand flower is remarkable for its size and striking colors. The individual blossoms are 3 to $4 \frac{1}{2}$ inches across, with 6 , and often 7 , divisions or lobes, and a large, richly veined throat of deep violet-red, passing into a beautiful indigo towards the orifice. This color gradually disappears and the pure-white outer ground is margined with innumerable dark-blue dots. Another strong point in its favor is that it nearly always comes true from seed. Per pkt., 30 cts.

\section{NEW HYBRID IMPATIENS SULTANI (BALSAMS).}

These new hybrid balsams are offered this year for the first time, and will, we believe, find favor with all flower-lovers. The plant is of an erect free-growing habit and the flowers are borne in great profusion until frost. They are of varying shades from bright-red to delicate pale-pink. They are very superior to the ordinary Sultani Balsams and are a great acquisition. Per pkt., 20 cts.

\section{IMPATIENS SULTANI (BALSAM), "KING ALBERT."}

This is one of the varieties described above, of a beautiful shade of rosy crimson, and produced in great profusion. Per pkt., $20 \mathrm{cts}$.

\section{SCHIZANTHUS PINNATUS NIVEUS.}

For the first time a pure-white Schizanthus is offered. It is true that white flowers havebeen produced by Schizanthus pinnatus albus and Schizanthus grandiflorus albus, but the former are spotted with blue and the latter with yellow. We are confident that this will find favor and prove a valuable addition. Per pkt., I5 cts.

\section{PAPAVER GLAUCUM (TULIP POPPY).}

A magnificent new Poppy recently discovered in the mountains of Armenia. The plant rises. to a height of $\mathrm{I} 2$ to $\mathrm{I} 4$ inches and produces above the bluish-green foliage a profusion (often 50 to 60) of large and splendid flowers of the most brilliant and vivid scarlet imaginable. The two outer petals bear a resemblance to a saucer, in which the two inner petals stand nearly upright and as if protecting the stamens. As the flowers fade, two black spots are visible at the base of the petals. The color reminds one of the bright scarlet of the Scarlet Duc van Thol Tulip, and on this account. the variety has been named the Tulip Poppy.

This will prove to be one of the very best decorative annuals, its dazzling-red flowers, produced in such profusion, forming a brilliant mass not to be excelled, and serving to brighten up other plants. with flowers or foliage of a more somber color. The minute seed will sometimes remain for two weeks before germinating, but if planted in a hot-bed it will germinate in about eight days. Per pkt., $20 \mathrm{cts}$. 


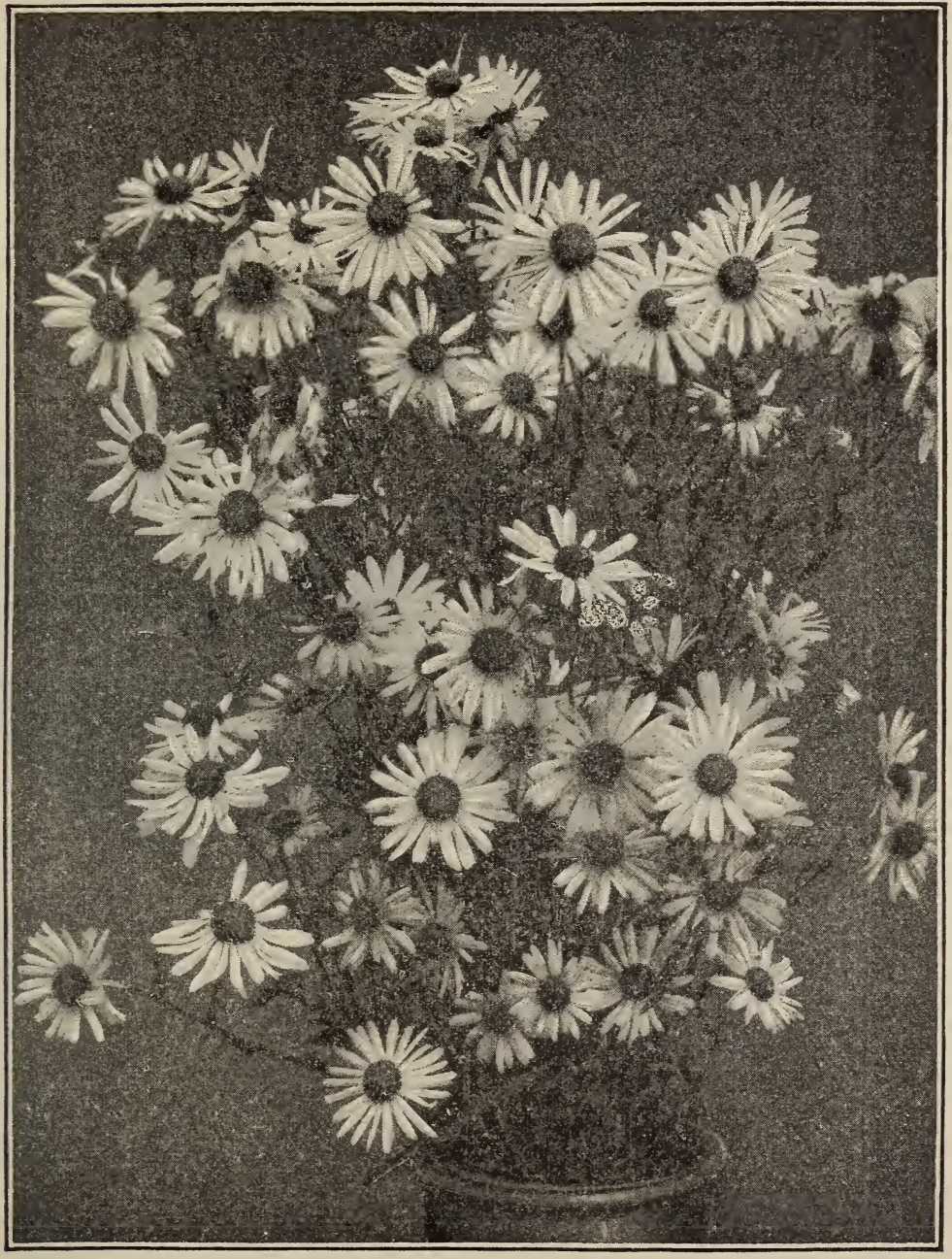

Pyrethrum Uliginosum.

For Seed, see Page 21. 


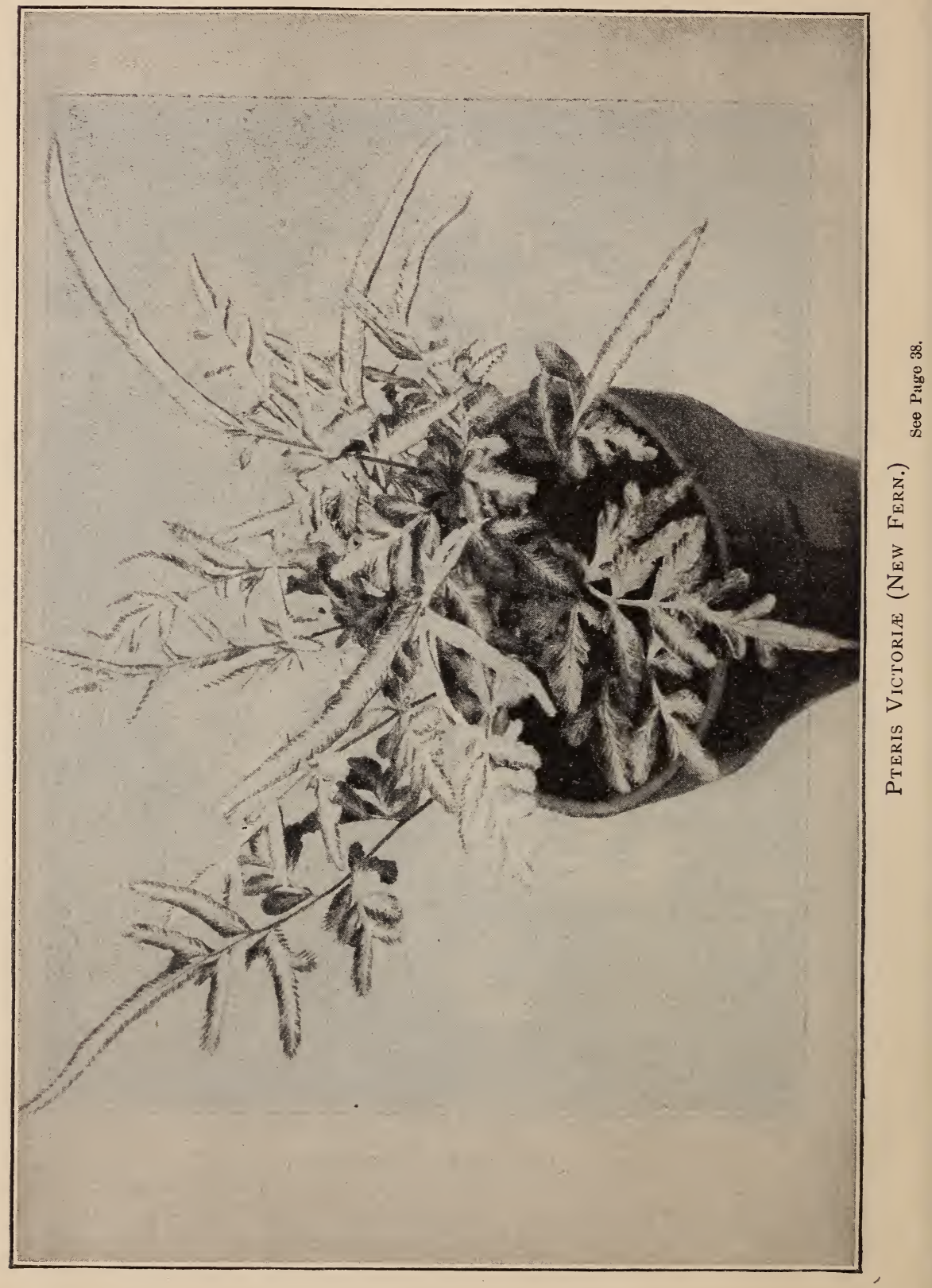




\title{
CHOICE FLOWER SEEDS IN BULK.
}

\author{
For Florists' and Dealers' Use.
}

The following list of Flower Seeds will be found to contain the chief kinds most in demand by Florists and Dealers. A more complete list is furnished in our retail catalogue.

On all orders for packets taken from our retail catalogue, for varieties therein quoted, we will allow a discount of $331 / 3$ per cent. When kinds are wanted in bulk we will furnish at advertised rates. When prices are quoted at 20,25 and 50 cents per ounce, not less than half an ounce will be sold; for varieties quoted at 75 cents and $\$ 1.00$ per oz., we will furnish quarter ounces, $\$ 1.50$ and above, per ounce, $1 / 8$ of an ounce will be sold at prices quoted; $1 / 4$ pound at pound rates.

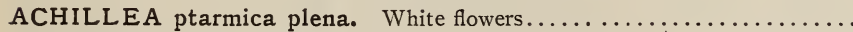

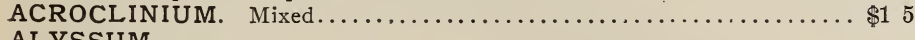

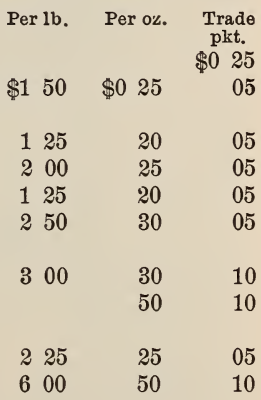

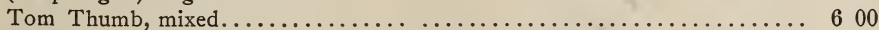

\section{ASTER-}

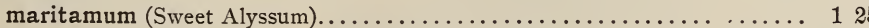

compactum. Very dwarf, pure white.............. 200

saxatile. Perennial Alyssum, yellow...................... 125

\section{AMPELOPSIS-}

compactum. Very dwarf ..................... 250

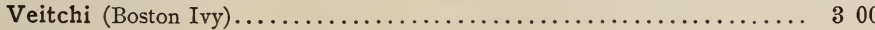

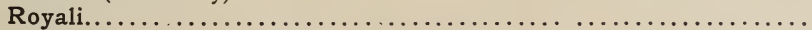

ANTIRRHINUM-

Dwarf Pyramidal Bouquet. White, Rose, Purple, Blue. Separate colors.

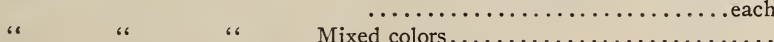

"Chrysanthemum-Flowered. Rose, White, Light Blue, Fiery Scarlet. Separate colors....... each

" Mignon. Pure White. Acknowledged to be the best variety of white

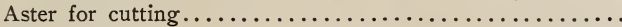

Princess. Pure White or Snowball. Somewhat of the Mignon type, resembling a white Pompone Dahlia; very long bloomer; excel-

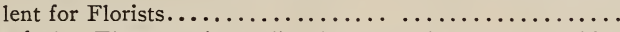

Queen of the Market. Three weeks earlier than any other Aster. White, Rose, Crimson, Light Blue and Dark Blue...

Quilled German Double (China). Mixed............................... White
Truffaut's Pæony-Flowered Perfection. White, Rose, Scarlet, Light Blue, Dark Blue. Separate colors..........each

Collections of all above kinds in 12 separate colors................

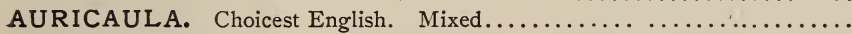
AQUILEGIA-

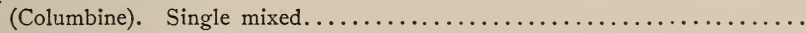

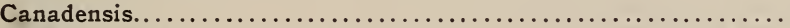

BEGONIA. Tuberous rooted-See Novelties.

Rex. Ornamental leaved................200 seeds, 50 cents.

Semperflorens rosea......................

BALSAM-

alba ........................ $1-32$ oz., 50

Double White (Camellia Flowered). Our strain of this most useful florist's

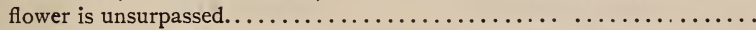

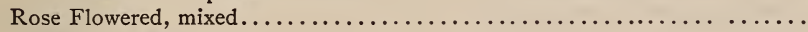

Camellia Flowered, choice mixed. 


\section{FLOWER SEEDS-Continued.}

BELLIS-

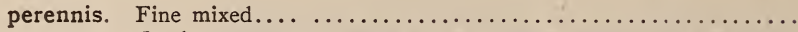

"f. p1. Snowball. Flowers very large and double, excellent

for bouquets................... $1-16$ oz., $\$ 100$.

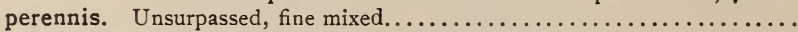

Longfellow. Similar in form and habit to Snowball, but of a fine dark rose color.

$\begin{array}{rrr}4 & & 50 \\ 5 & & 20 \\ 5 & & \\ & & 25 \\ 50 & & \\ 50 & 10 \\ 50 & 10 \\ 60 & 10 \\ 50 & 10\end{array}$

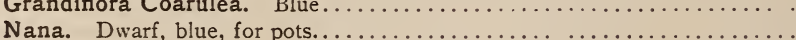

CALCEOLARIA-

hybrida grandiflora. Self-colored varieties.. . ........1-16 oz., $\$ 250$.

Pumila compacta. Dwarf Tigered.................. $1-32$ oz., $\$ 250$.

rugosa. Shrubby or bedding.....................

CALENDULA. Prince of Orange, golden yellow................. 100

CALLA æthiopica..................................... 300

CALLIOPSIS. (Coreopsis).

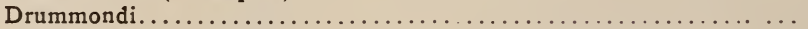

Lanceolata. Own saving. . . . . . . . . .

Choice mixed...................................... 100

CANDYTUFT-

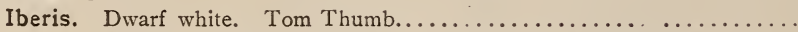

affinis. Empress. Very large trusses of pure white flowers......

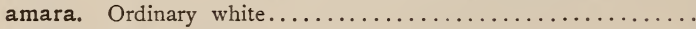

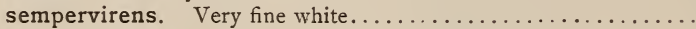

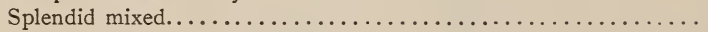

\section{CANNA-}

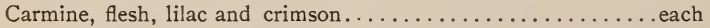

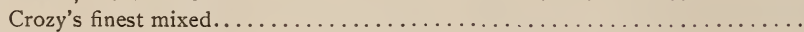

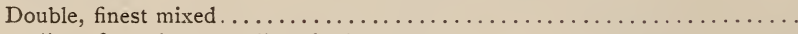

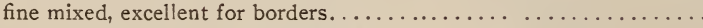

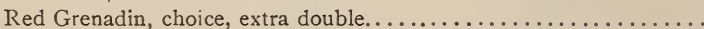

Double Marguerite. This new class has justified the highest expectations. Seedlings begin blooming the fourth month from time of germinating; they supply the florist or gardener with large and very sweetly scented flowers when other carnations are over. Eighty per cent. are double and produce a rich display of colors. With the aid of this class the florist can have carnations all the year round. Those who used it last year are high in its praise....................1-16 oz., 50 cts.

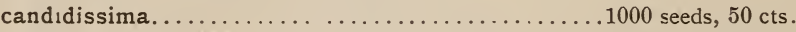

gymnocarpa.................... " 25 cts.

Clementei.......................... 50 cts.

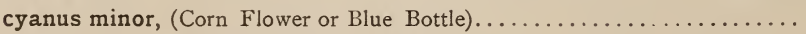

CHRYSANTHEMUMS. (See Novelties).

Double Japanese and Chinese saved from our prize specimens only....... 15c. per pkt.; $\$ 150$ doz. packets. .

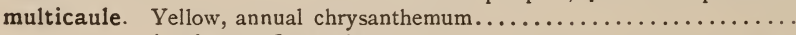

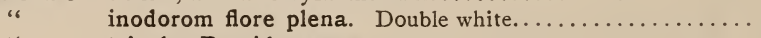

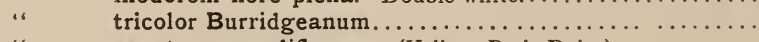
" segetum grandiflorum. (Yellow Paris Daisy)............

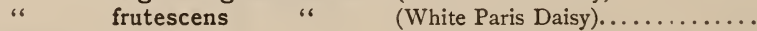




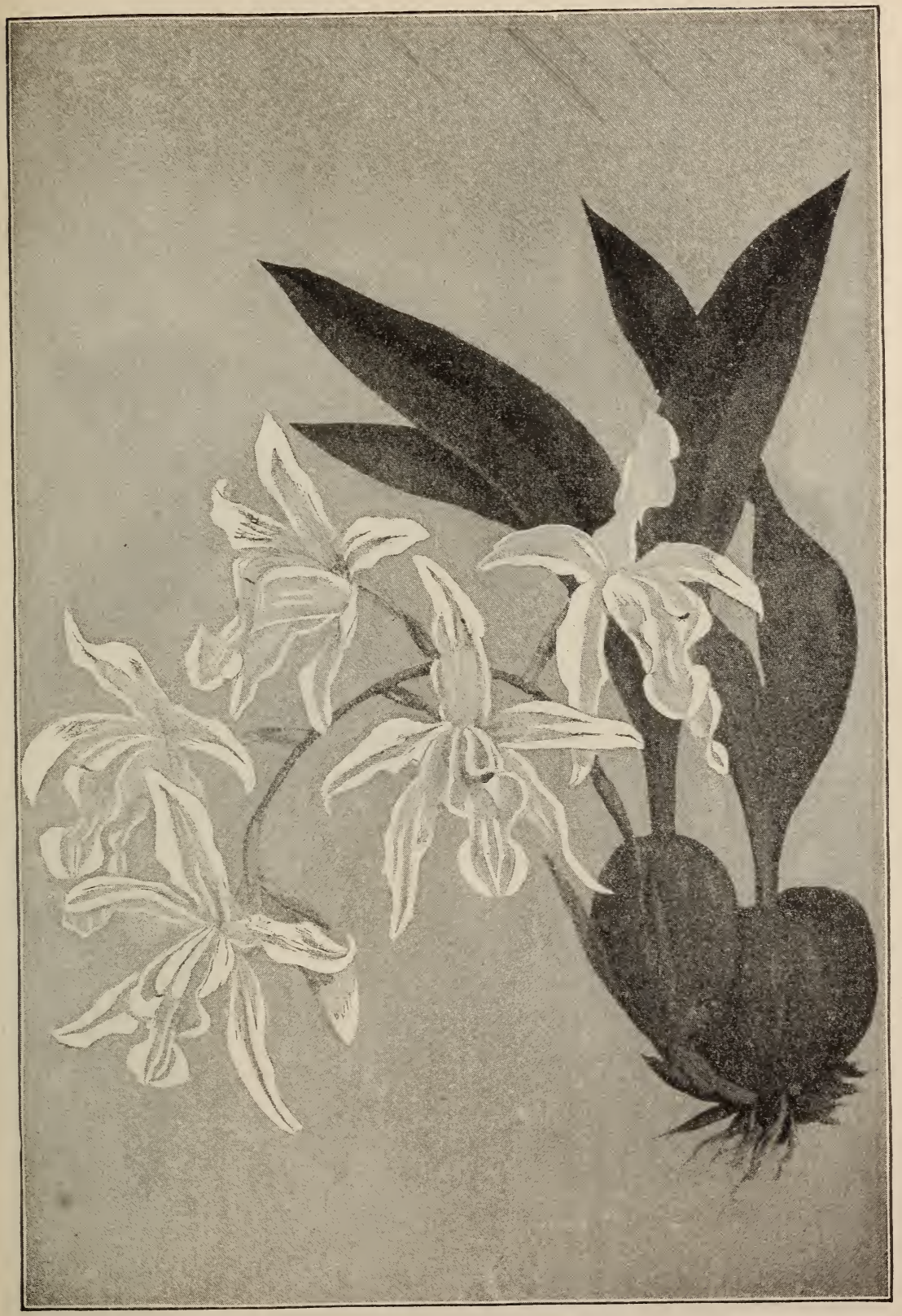

Celogyne Cristata.

See Page 54. 


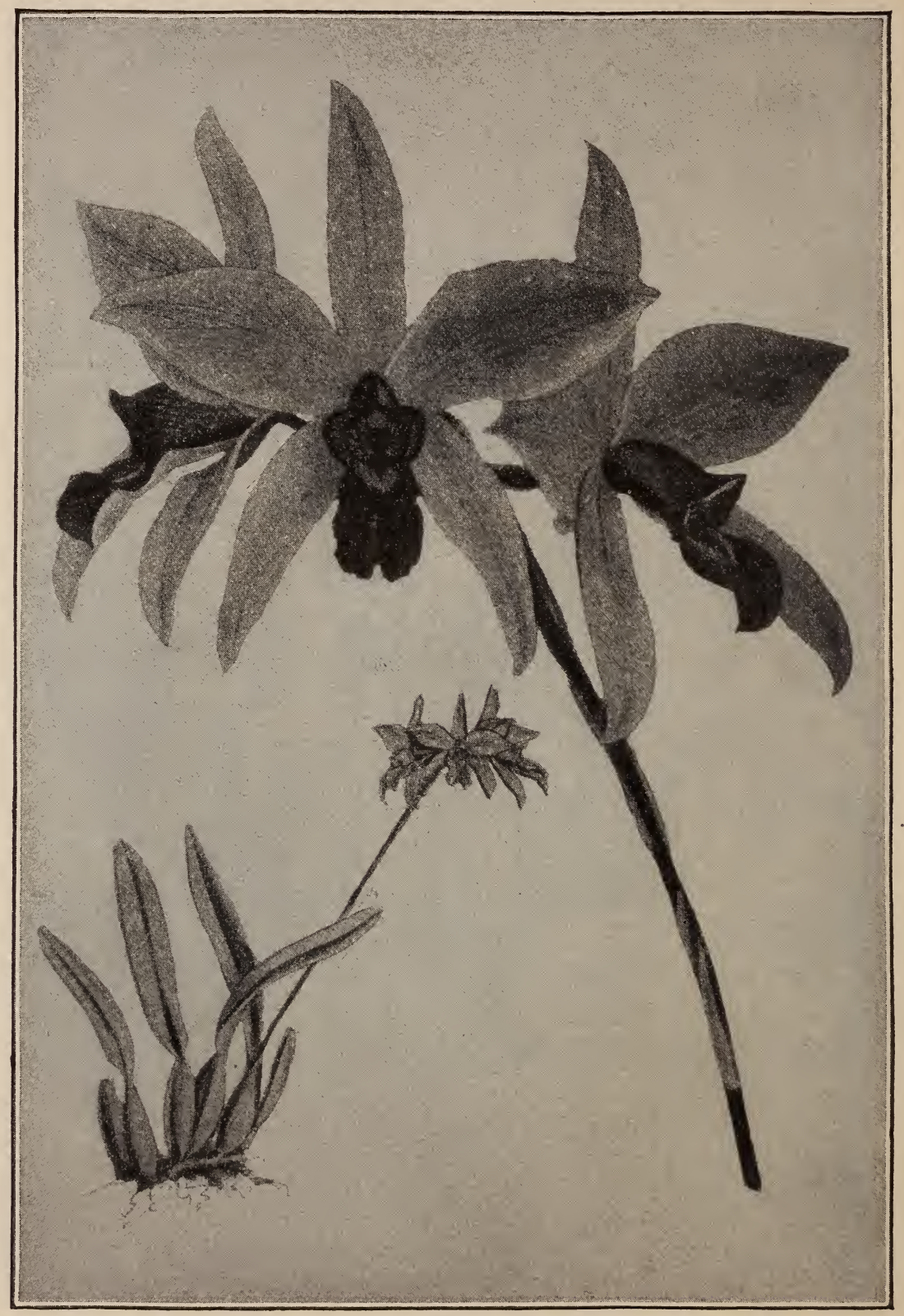

LÆlia Anceps.

See Page 54. 
FLOWER SEEDS-Continued.

COCKSCOMB (Celosia.)-

Plumosa Aurea. Yellow feathered.................. per $1-16$ oz. $50 \mathrm{c}$.

Perlb. Per oz. $\quad \begin{array}{r}\text { Trade } \\ \text { Plt. }\end{array}$

" " Scarlet feathered................ "50c.

Glasgow Prize, crimson and yellow, very choice strain $\ldots \ldots \ldots \ldots \ldots \ldots$.

CONVOLVULUS-

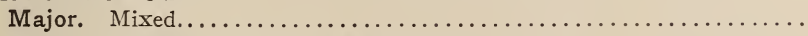

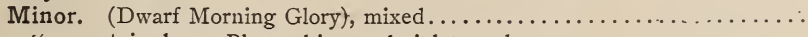

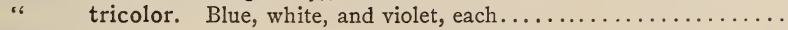

\section{cosmos}

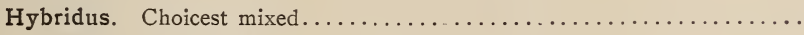

\section{CYCLAMEN-}

persicum giganteum album. White...100 seeds, $\$ 125 ; 1000$ seeds, $\$ 10$. atrorubrum. Dark red. 100 seeds $\$ 125$. 1000 “ $\$ 1000$.

$\begin{array}{llll}40 & 10 & \\ 40 & 10 & \\ 40 & 10 & \\ & & & \\ 600 & 50 & .10 \\ 700 & 75 & 10\end{array}$

Mixed...........100 seeds, $\$ 100 ; 1000$ seeds, $\$ 8$.

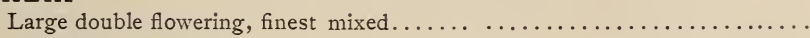

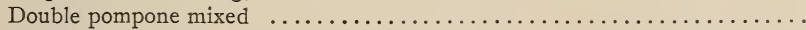

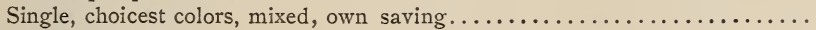

DELPHINIUM-

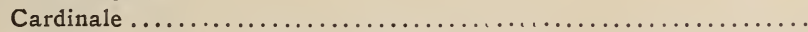

formosum. The seed we offer of this grand perennial is our own saving..

DIANTHUS-

Barbatus. (Sweet William). Choice strain of our own saving..........

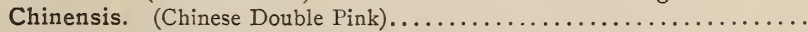

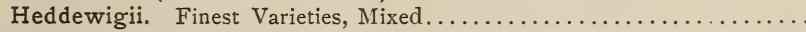

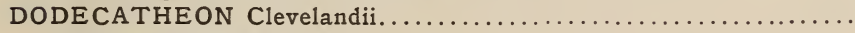

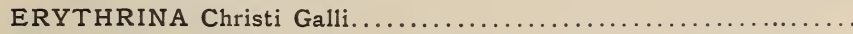

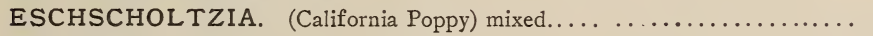

\section{FUCHSIA-}

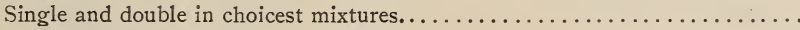

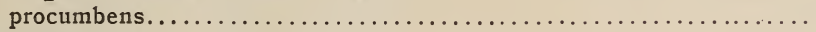

GERANIUM-

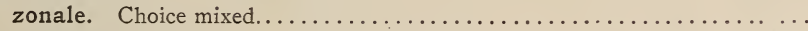

Apple scented.............................

GLOXINIA. Choice mixed. Our own saving, choice strain............

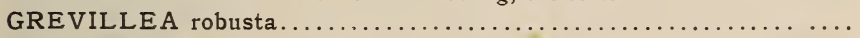

\section{GYPSOPHILA-}

muralis

HE

HELIOTROPE. Fine mixed $\ldots \ldots \ldots \ldots \ldots \ldots \ldots \ldots \ldots \ldots \ldots \ldots \ldots \ldots \ldots$
HOLLYHOCK -

Choicest double strain. Crimson, yellow, whit3, magenta; separate colors, each.

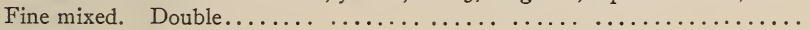

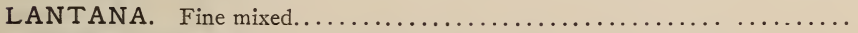

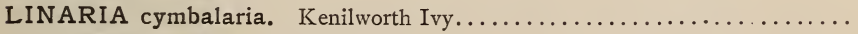

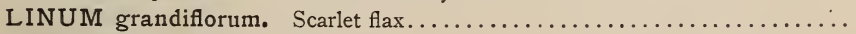

LOBELIA-

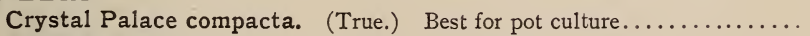

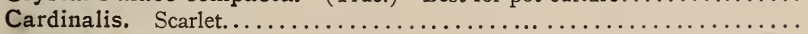

LYCHNIS. Choice mixed... . . . . . . . . . . . . . . . . .

MARIGOLD-

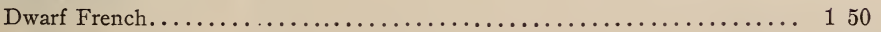

$200 \quad 10$

$400 \quad 25$

$150 \quad 20$

$100 \quad 10$

$300 \quad 30 \quad 10$

$100 \quad 10$

25

$60 \quad 10$

$200 \quad 15$

$100 \quad 10$

$125 \quad 20 \quad 5$

50

50

$125 \quad 25$

$\$ 0 \quad 50$

$\$ 100 \quad 10$

$30 \quad 05$

$25 \quad 05$

$125 \quad 20$

$100 \quad 20$

$100 \quad 20$

$25 \quad 05$

$200 \quad 20$

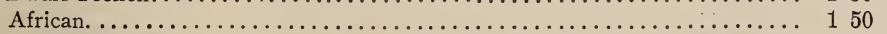

MAURANDIA-

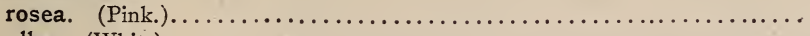

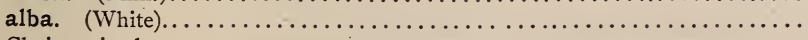

Choice mixed. . 


\section{FLOWER SEEDS-Continued.}

MIGNONETTE-

Large flowering.

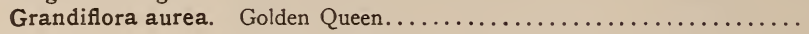

Machet.

Crimson Queen ......................... 300

Miles' Spiral. Best for cut flowers

dissitiflora. (Forget Me Not). Blue. Useful for florists.............. semperflorens. Especially useful for cut flowers. Blooms all year round. NASTURTIUM-

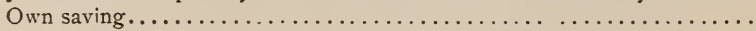

Dwarf or Tom Thumb. Scarlet.

"Empress of India. The very best variety for florists ; flowers bright

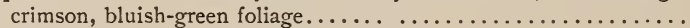

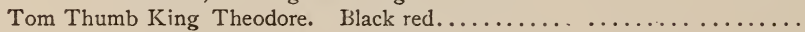

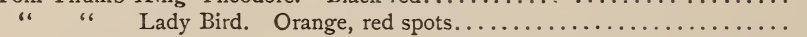

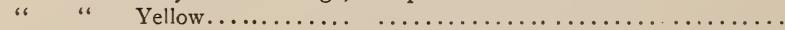

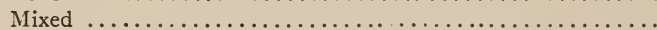

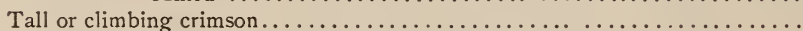

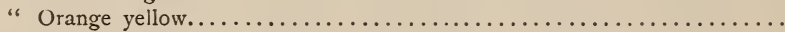

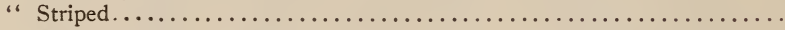

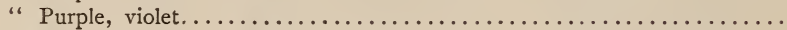

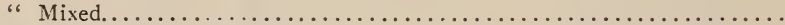

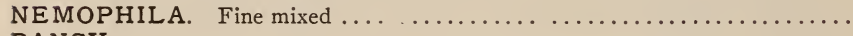
PANSY-

Very fine mixed.................................... 600

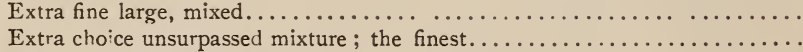

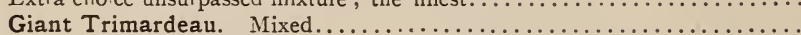

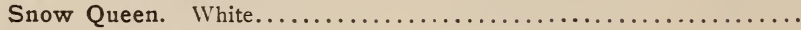

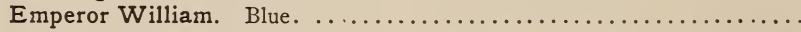

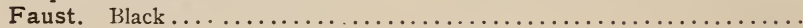

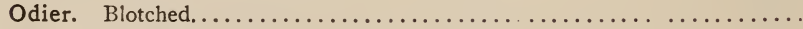

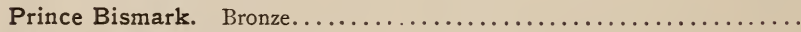

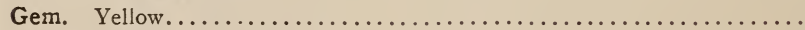

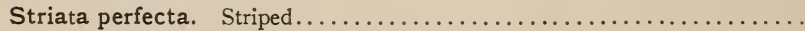

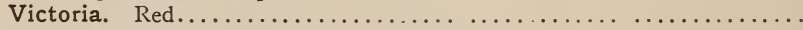

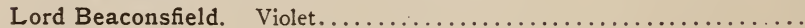

PEAS, Sweet.

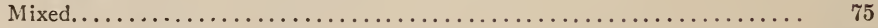

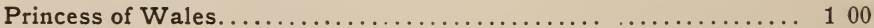

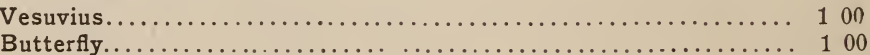

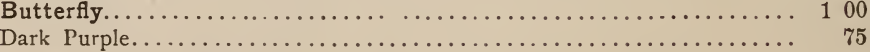

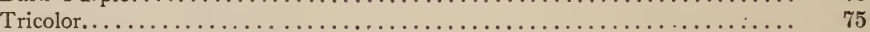

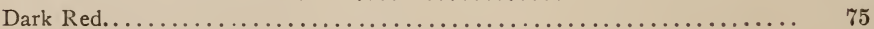

Invincible Scarlet.................................. 75

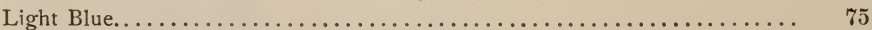

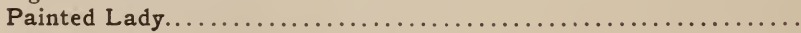

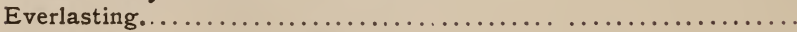

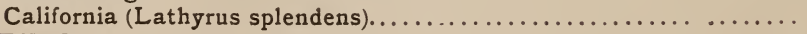

PETUNIA-

hybrida. Very fine mixed.......................... 600

Starred and Striped....................................... $\$ 1$

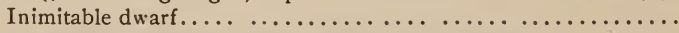

Grandiflora fimbriata flora plena. Fine mixed. ............ 


\section{FLOWER SEEDS-Continued.}

\section{PORTULACA Grandiflora.}

$\begin{array}{rrrr}\text { Per lb. } & \text { Per oz. } & \begin{array}{c}\text { Trade } \\ \text { pkt. }\end{array} \\ \$ 2 & 50 & \$ 025 & \$(10 \\ 300 & 30 & 10 \\ 300 & 30 & 10 \\ 300 & & 30 & 10 \\ 300 & & 30 & 10 \\ 300 & & 30 & 10 \\ 300 & & 30 & 10 \\ 300 & & 30 & 10 \\ & & 500 & 50\end{array}$

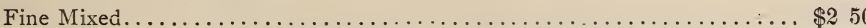

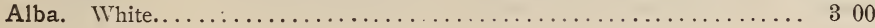

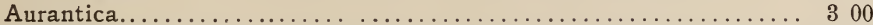

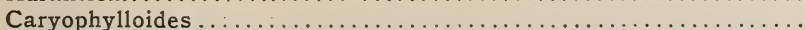

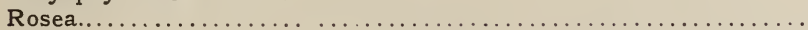

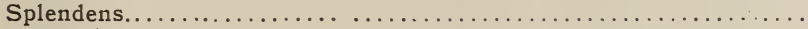

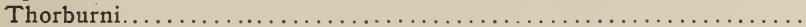

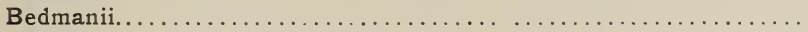

Double mixed, best.

PHLOX Drummondii Grandiflora-

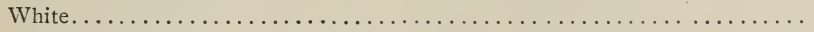

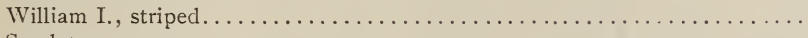

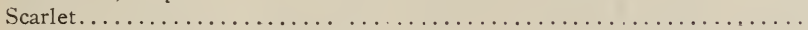

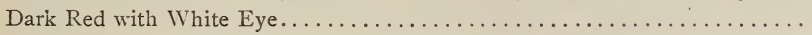

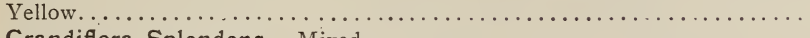

Grandiflora Splendens. Mixed....................... 800

\section{Drummondii-}

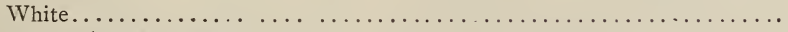

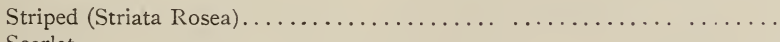

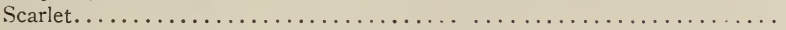

Dark Red (Atropurpurpea) . . . . . . . . . . . . . . . . . . . .

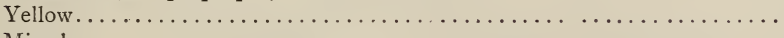

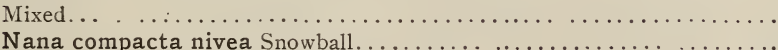

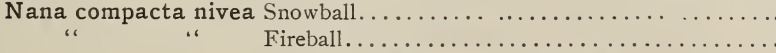

Mixed. The best for pot culture...........

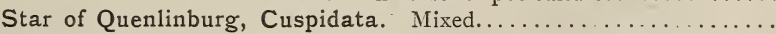

\section{PRIMULA-}

sinensis fimbriata. Choicest mixed............. 1-64 oz., $\$ 075$.

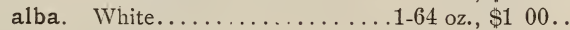
rubra. Red................ $1-64$ oz., $\$ 075$.

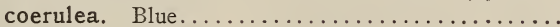
coccinea. Scarlet............64 oz., $\$ 100 \ldots$

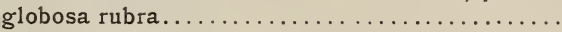

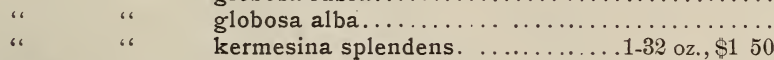

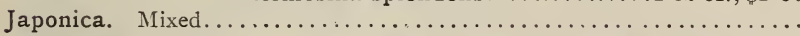
obconica. White flowers. Very useful for pots and bouquets, 1-64 oz., $\$ 1$.

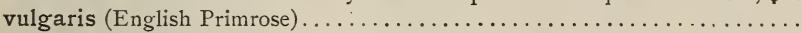

PYRETHRUM-

aureum (Golden Feather) . . . . . . . . . . . . . . . . . . . .

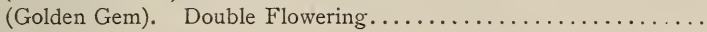

ROMNEYA Coulteri..................................... 1000

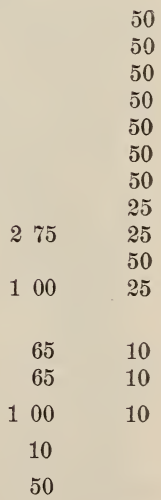

SMILAX

Double Ten-Weeks, Large-flowering Dwarf. White, Scarlet, Blue,

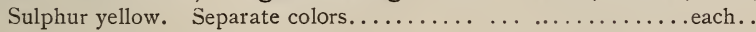

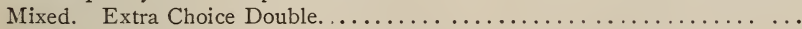

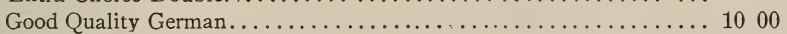

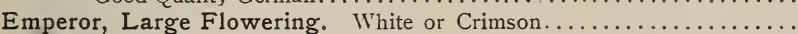

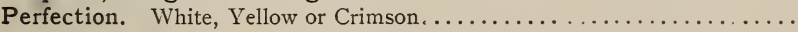

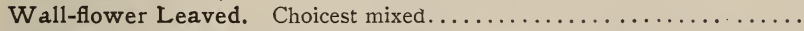

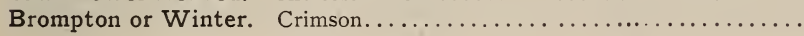

$500 \quad 25$

$50 \quad 10$

$200 \quad 20$

" White.

$100 \quad 10$

Dwarf Bouquet Winter, Crimson.

$500 \quad 25$

$500 \quad 25$ ، 


\title{
FLOWER SEEDS-Continued.
}

TASCONIA Buchenani.... . . . . . . . . . . . . . . . . . . . . .

TORENIA Fournieri. Charming plant for pot culture : velvety blue fowers..
VERBENA-

Per lb. Per oz. Trade

$\$ 0 \quad 75 \$ \$ 10$

New Mammoth

Hybrida. Scarlet.

400

Blue with white eye...........................

\section{Hardy Perennial Seed.}

\author{
OF OUR OWN SAVING.
}

Growing, as we do, many acres of these most useful and pretty plants, for which there is a "steadily increasing demand, assisted by a favorable season, we have saved a quantity of seed of the most important kinds, and offer below a list at prices which will compare favorably with those of the European growers; but when taken into consideration the certainty of germination and extra care with which they are saved, they should receive the preference.

\section{Hardy Perennial Seeds.}

$1.8 \mathrm{Oz} .1 \mathrm{Oz}$.

Achillea tomentosa. Dwarf yellow. $\$ 0 \quad 75 \$ 500$

Aconitum anthora. Yellow... ... 25

Agrostemma-

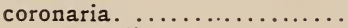

alba............... 50

Anemone-

patens Nuttalliana......... 50

Pennsylvauica...............

sylvestris ................

Aquilegia-

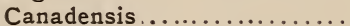

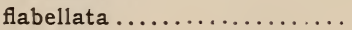

vulgaris alba..............

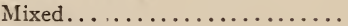

Arabis albida................

Aristolochia elegans.......... 100

Asclepias-

tuberosa (Rubbed Seed)....... 25

Aster-

Incarnata (Rubbed Seed)..... 25

Nuvae Belgii............. 25

Novae Angliae............... 25

Ptarmicoides (Rubbed Seed)... 25

Sibericus ............... 25

Douglasii .................... 25

prenanthoides ............ 25

laevigatus .............. 25

cordifolius ............... 25

niveum .............. 25

spectabilis .............. 25

puniceus ................ 25

Lindleyana............ 25

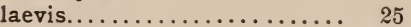

diffusus $\ldots \ldots \ldots \ldots \ldots \ldots \ldots \ldots$ 25
Aster- $\quad 1-8 \mathrm{Oz} .1 \mathrm{Oz}$.

multiflorus ............. $\$ 025$

Shortii.................. 25

Henryii................ 25

Drummondii............. 25

surculosus.............. 25

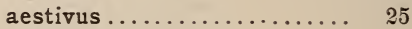

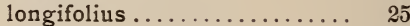

white................. 25

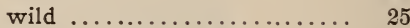

Astragalus Canadensis........ 25

Aubrietia-

Eyrei................. 25

Graeca .................. 25

Baptisia laucantha. Yellow..... 50

Boltonia asteroides........... 25

Callirhoe involucrata........... 50

Campanula-

Carpatica................. 25

celtidifolia ................ 25

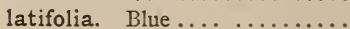

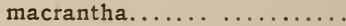

Persicifolia............... 30

Punctata................. 25

Chelone nemorosa........... 50

Chionodoxa-

gigantea............... 50

Lucillea ................ 50

Sardinensis............... 50

Clematis alpina. Erect white.... 25

Corchoropsis crenata.......... 25

Dahlia. Single (Own grand strain). $\quad 25$ 


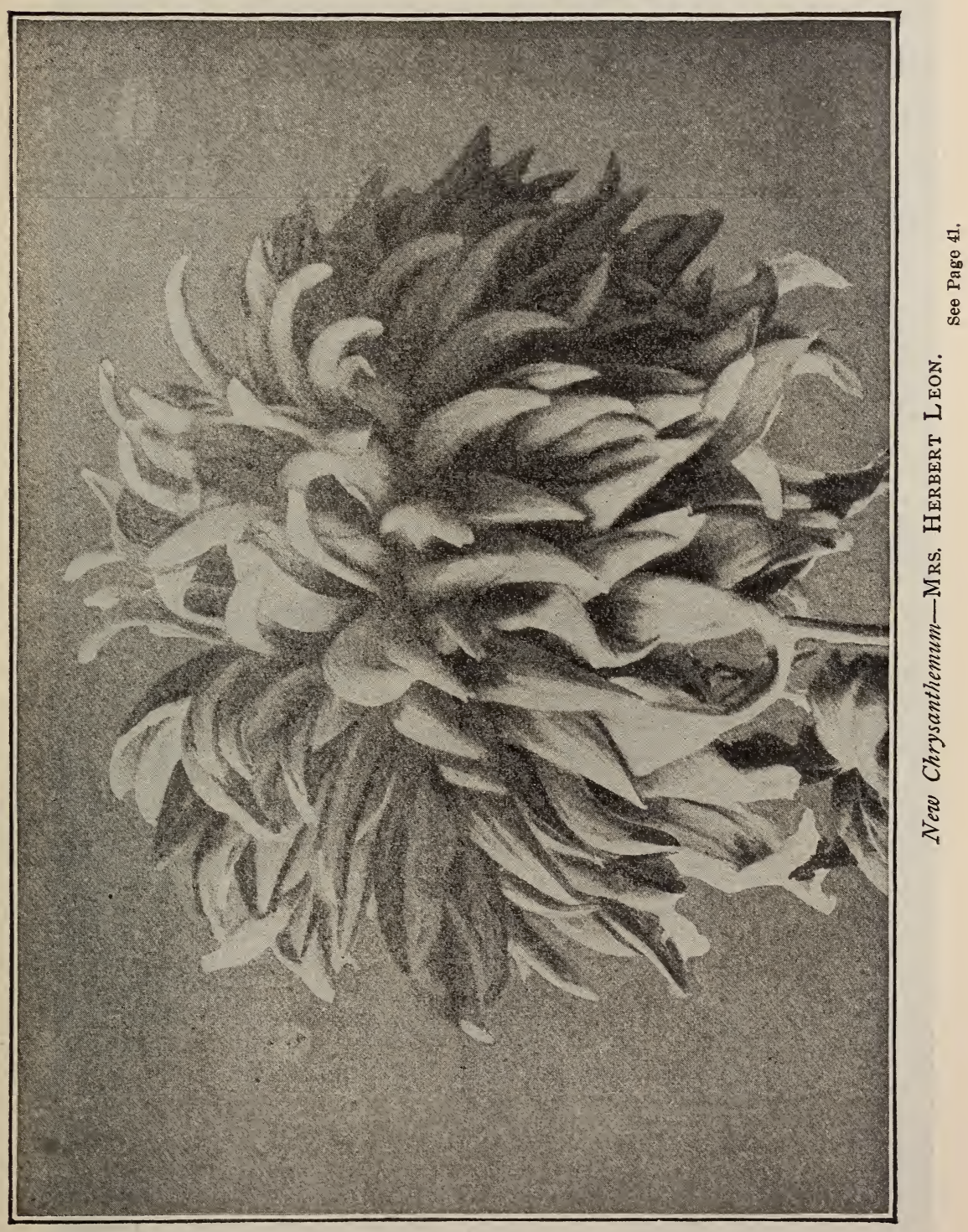




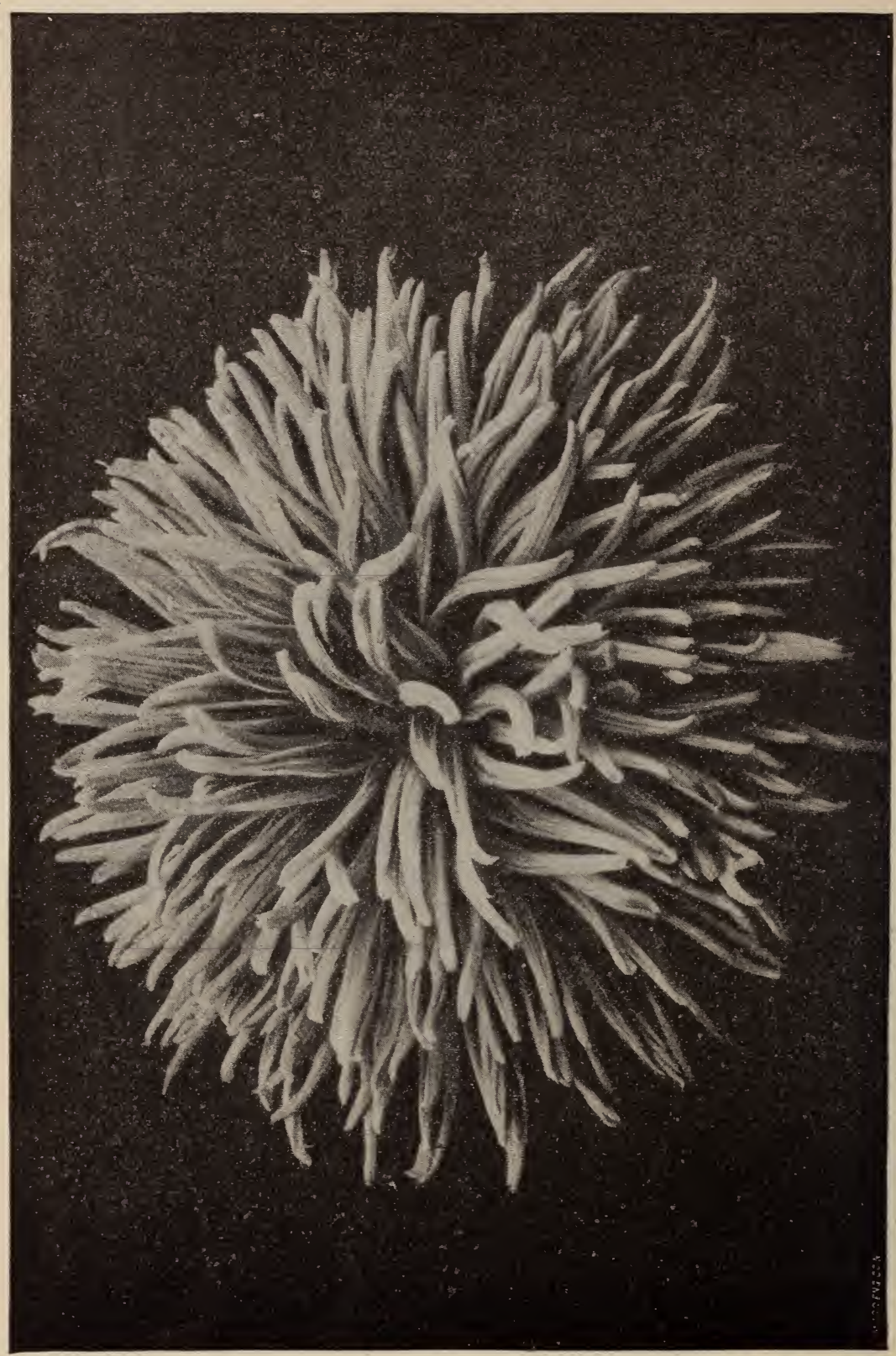

: 
HARDY PERENNIAL SEEL-Continued.

Delphinium hybridium. Mixed. Single and double......

Dianthus-

barbatus....................

hispanicus. Dark red, large flower................... deltoides..................

Dictamnus-

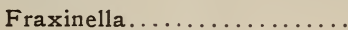

alba..............

Digitalis-

gloxinioides. Mixed........

alba......................

purpurea.....

rosea.........

Erigeron-

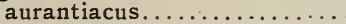

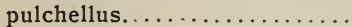

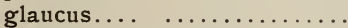

Eryngium amethystinum.......

Erysimum pulchellum..........

Eupatorium species. White.....

Funkia-

lanceolata................

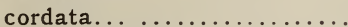

Gentiana Andrewsii...........

Geranium maculatum..........

Geum-

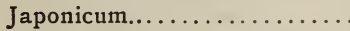

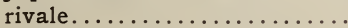

Grindellia hirsuta.............

Gypsophila repens ............

Helenium autumnale...........

Helianthus-

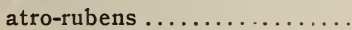

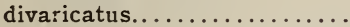

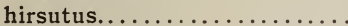

giganteus .............

Hemerocallis-

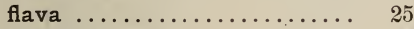

graminea................ 35

rutilans................ 35

Thunbergii ............ 35

Hibiscus rose.............. 25

Hollyhock. Single............

Iris-

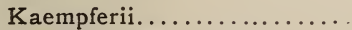

pseudo acorus. Yellow.......

Siberica ................. " sanguinea.........

Lathyrus niger. Very dark purple.

Lepachys pinnata. Drooping, very pretty, yellow, black centre....

Liatris spicata..............

Lilium-

Carniolicum.

5

Polemonium coeruleum.........

Martagon. .

Pyrenaicum..............

Szovitzianum.

Linum-

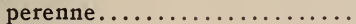

Austriacum.

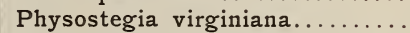

Potentilla fruticosa.... . ......

Poterium canadense............

Pycanthemum lanceolata.......

Pyrethrum-

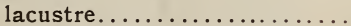

cinerariaefolium ............ uliginosum. The giant white

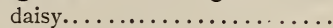

Reseda glauca. White perennial.

Rudbeckialaciniata.

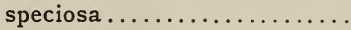

Ruellia ciliosa............... 25

Salvia pratensis. Hardy blue.... 25

Sanguinaria Canadensis ....... 25

Scutellaria scordifolia.... ...... 50

Silphium perfoliatum..........

Solidago-

Canadensis. (Golden Rod or

1.8 Oz. $\quad 1 \mathrm{Oz}$. $\$ 100$

American National flower) ... 50

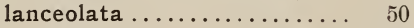
Drummondii ............ 50 occidentalis.............. 50 Odora ................ 50 juncea............... 50 neglecta .............. 50 serotina............... 50 rigida. The best of the Solidagos, being dwarf in habit and much larger flowers............ 100

Tunica Saxifraga............. Verbascumthapsus.............. 25 niger................. 25

Veronicaspicata................ 25 rigida. (Rubbed Seed)....... 200 virginica............. 25

25




\title{
BULBS
}

-FOR-

\section{SPRING PLANTING AND SUMMER BLOOMING.}

\begin{abstract}
AGAPANTHUS Umbellatus. (African Lily or Love Flower)....... AMARYLLIS-

Atamasco (Zephyranthus Atamasco). (Flower of the West Wind)...

Aulica (Hippeastrum aulicum). (Lily of the Palace), winter bloomer...\$1 25

Belladonna Major (Belladonna Lily). Autumn blooming species.... 25

Cinnamonea (Crinum riparium). Splendid rose color......... 500

Formosissima (Sprckelia formosissima). (Jacobean Lily), good for forcing.................................... 15

Gigantea (Brunsvigia Josephinex). Beautiful and rare species..... 500

Johnsonii (Hippeastrum Johnsonii). Crimson, striped white...... 75

Ornatus. Grand hybrid; white flower, striped with rose......... 700

Reginæ (Mexican Lily). Dark red, with orange and white..........

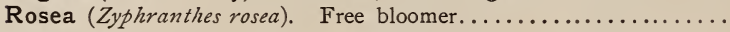

Purpurea (Vallotta purpurea). (Scarborough Lily), bright scarlet...

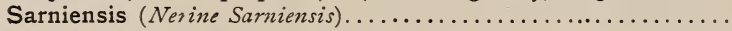

AMORPHOPHALLUS-

Campanulatus................................. 125

Giganteus.................................. 100

Rivieri. (Snake Palm or Umbrella Arum)................. 25 ANEMONE-
\end{abstract}

Each. Per doz. Per 100 Per 1000

Coronaria or Hortensis Varieties. Single, all colors, mixed..........

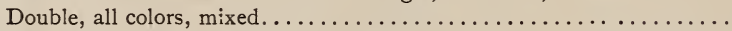

BEGONIAS, Tuberous Rooted. See Flowering and Foliage Plants...

CALADIUM Esculentum (Elephant's Ear). A great favorite.......

CALLA LILIES$\ldots \ldots \ldots \ldots \ldots 500, \$ 00$, and $\$ 1200$ per 100 .

White Calla (Richardia Aethiopica). (White Trumpet Lily or Lily of the Nile). Dry roots, ready August.....................

Spotted Calla, (Richardia albo-maculata). Dormant tubers...........

Yellow Calla. (Richardia hastata)........................ 100

$\$ 175$

$\$ 200=\$ 1500$

200

150

600

$300 \quad 2400$

$50 \quad 400$

300

$160 \quad 1200$

Black Calla. (Arum sanctum). Recently introduced............ 60

CONVALLARIA. See Lily of the Valley.

CRINUM Americanum. Fragrant white flowers................

CYCLAMEN-

Europæum roseum. Bright red, fragrant, hardy............. album. Pure white, "، " $\ldots \ldots \ldots \ldots \ldots \ldots \ldots$

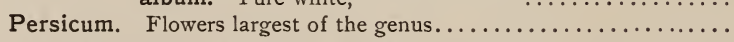

CYCLOBOTHRA Flava (Golden Shell). Mexican variety.......... EUCHARIS Amazonica (Lily of the Amazon). Greenhouse bulb.... FREESIA Refracta alba. Write us for price for July and August

$25 \quad 250$

$175 \quad 1000$

65500

1100

700.5000

$\begin{array}{llll}130 & 1000\end{array}$

$\begin{array}{lllll}130 & 10 & 00\end{array}$

$\begin{array}{lll}160 & 1200\end{array}$

$50 \quad 300$ delivery.

\section{GLADIOLUS.}

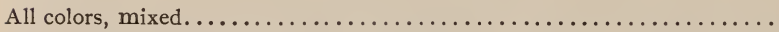

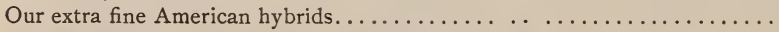

White and light. Extra choice for florists.........................

" " Choice mixture of seedlings and named varieties........

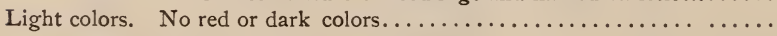




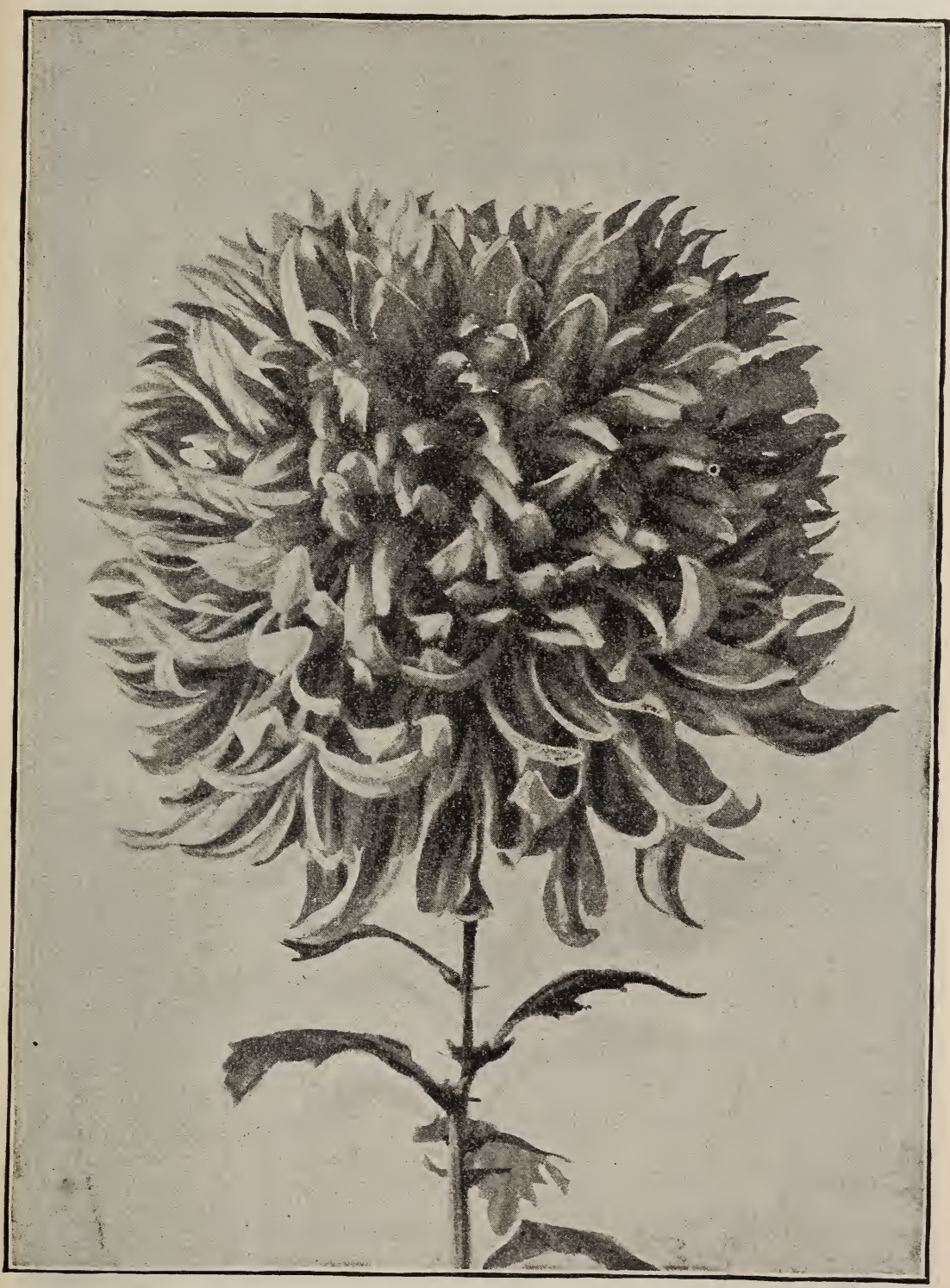

New Chrysanthemum-Dr. H. A. MANDERviLle. 


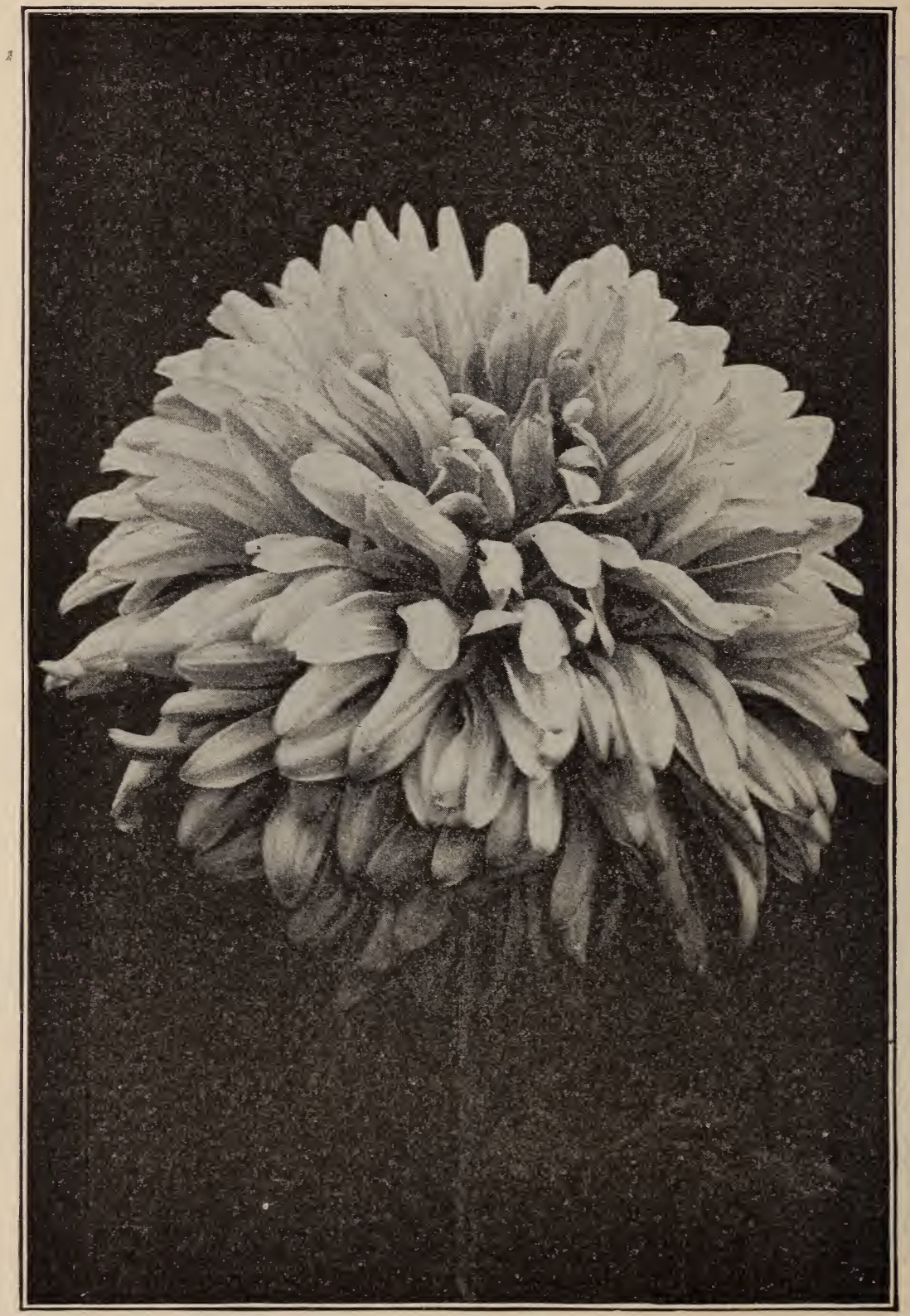

Nere Chrysanthemum-Mrs. W. S. Kimbali.

See Page 41 . 


\section{BULBS-Continued.}

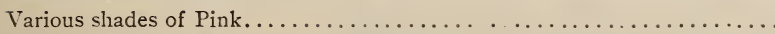

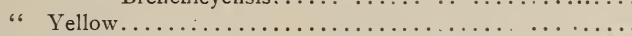

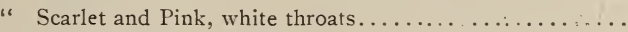

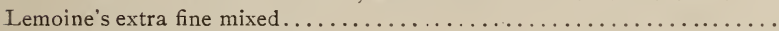

Fine mixed varieties $\ldots \ldots \ldots \ldots \ldots \ldots \ldots \ldots \ldots$

HEMORCALLIS. See Herbaceous Plants.

HYACINTHUS Candicans. (Cape Hyacinth). Flowers creamy

IRIS. See Herbaceous Plants.

\section{LILIES.}

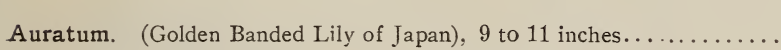

6

vittatum rubrum. Magnificent flowers, cream white......

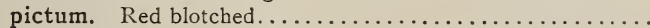

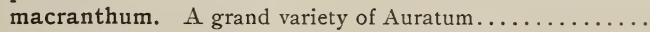
virginale alba. White with yellow stripe on each petal..... Wittei. Pure white with broad yellow band.............

Batemanni. Three or four feet high, flowers apricot color..............

Brownii (Japonicum odorum). Yellow and purple, very fine...........

Canadense (Canadian Lily). Yellow varying to pale red, spotted with

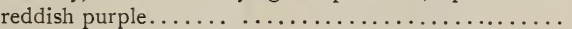

Candidum (Annunciation Lily, St. Joseph's Lily). The well-known white Lily of the gardens..................

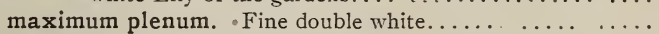

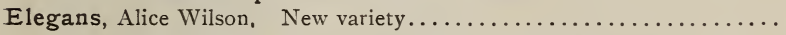

"Incomparable. Fine new variety, large flowers... . . . . . . .

، atropurpureum. Scarlet with black spots.............. Mixed varieties. . . . . . . . . . . . . . . . . . . . . . . . .

Hansoni (True Japanese Yellow Martagon). . . . . . . . . . . . . .

Harrisii (Bermuda Easter Lily). Write for our prices and sizes for delivery in July and August.

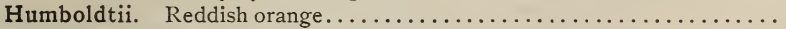

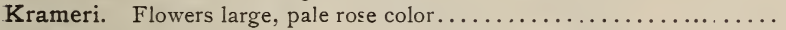

Longiflorum. Similar to Harrisii but makes a better pot plant. Prices upon application.

" foliis albis marginatis. Variegated leaves...........

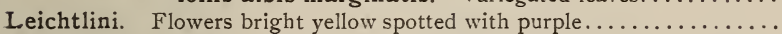

Martagon. Flowers purplish red with numerous spots of dark purple....

Maritimum. A dwarf grower, flowers blood red................

Maximowiczii. Bright scarlet, spotted with purple..............

Medeoloides. Orange red, with a few purple spots...............

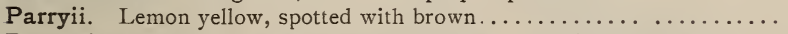

Pyrenaicum. Small, bright yellow; the petals much incurving.........

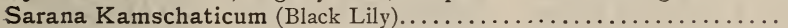

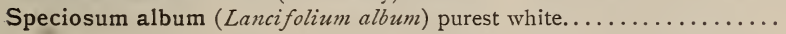
rubrum. White, shaded with deep rose, and spotted with red.

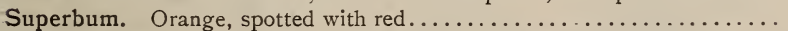

Szovitzianum (Monadelphum, colchicum). Pale bright yellow..........

Testaceum (excelsum, Isabellum). Yellow tinged with dull red..........

Tigrinum (Common Tiger Lily). Orange red, spotted with black........

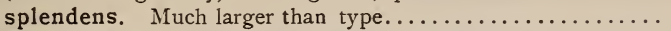
flore plenum. Semi-double form of Tigrinum...........

Washingtonianum (Shasta Lily). White, tinged with purple.......... " purpureum (rubescens). Whitish, with purple spots..
$1200 \quad 10000$

$\begin{array}{lllll}50 & 4 & 00 & 32 & 00\end{array}$

$\begin{array}{lllll}50 & 5 & 00 & 40 & 00\end{array}$

$50 \quad 500 \quad 4000$

$\begin{array}{lllll}50 & 5 & 00 & 40 & 00\end{array}$

$\begin{array}{lllll}75 & 7 & 50 & 60 & 00\end{array}$

$\begin{array}{lllll}10 & 1 & 00 & 8 & 00\end{array}$

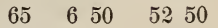

\begin{tabular}{ll|l}
$10 \quad 80$ & 600
\end{tabular} 
LILIES-Continued.

LILY OF THE VALLEY (Convallaria majalis). Almost at any time of the year we can furnish strong, three-year-old Berlin pips in

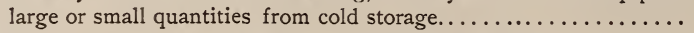

MADERIA Vine. The old favorite "Climbing Mignonette".........

MILLA Biflora. (Mexican Star Flower). Fine for forcing...........

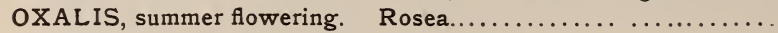

PAEONIA. See Herbaceous Plants.

PANCRATIUM-

Calathinum. Large flowers, for pot culture...............

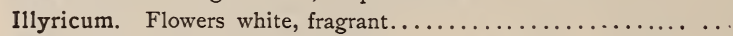

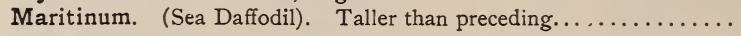
RANUNCULUS,

Double Persian. Rose-shaped flowers. All colors mixed.........

Double French. Larger flowers. All colors mixed..............

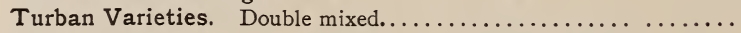

SCHIZOSTYLIS Coccinea (Kaffir Lily). Greenhouse plant........ TIGRIDIA-

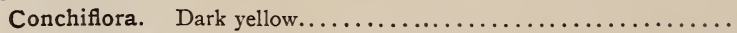

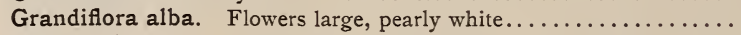

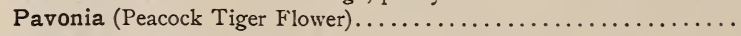

TRITOMA Uvaria Grandiflora (Red-hot Poker Plant).............

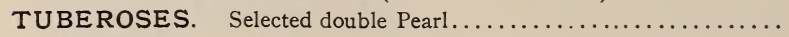

Each Per doz. Per 100 Per 1000 $\begin{array}{rrrrr}\$ 0 & 30 & \$ 2 & 50 \\ 50 & & 3 & 50 \\ & & & \\ 10 & & 50 \\ & & & & \\ & & & & \\ 2 & 00 & & 15 & 00 \\ 3 & 50 & & 25 & 00 \\ 2 & 00 & & 15 & 00\end{array}$

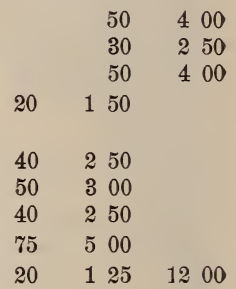

\title{
DON'T
}

Place your Order Before Receiving Our Pricesion

\author{
BERMUDA EASTER LILY BULBS, \\ LILIUM LONGIFLORUM BULBS, \\ ROMAN HYACINTH BULBS, \\ DUTCH HYACINTH BULBS, \\ FORCING TULIP BULBS, \\ PAPER WHITE NARCISSUS BULBS, \\ VON SION NARCISSUS BULBS \\ LARGE FREESIAS, DOUBLE EXTRA SIZE BULBS. \\ LILY OF THE VALLEY PIPS,
}

CALLA LILY, DRY BULBS, WHITE, YELLOW, BLACK AND SPOTTED

CALla iLIL, DWARF DRY BULBS, NEW NOVELTY, “ThE GEM." -ALSO ALL-

\section{FRENCH, DUTCH AND AMERICAN BULBS.}

\section{OUR EXCEPTIONAL OFFER IS}

EXTRA LARGE, SELECT BULBS, UNEQUALLED LOW PRICES, 


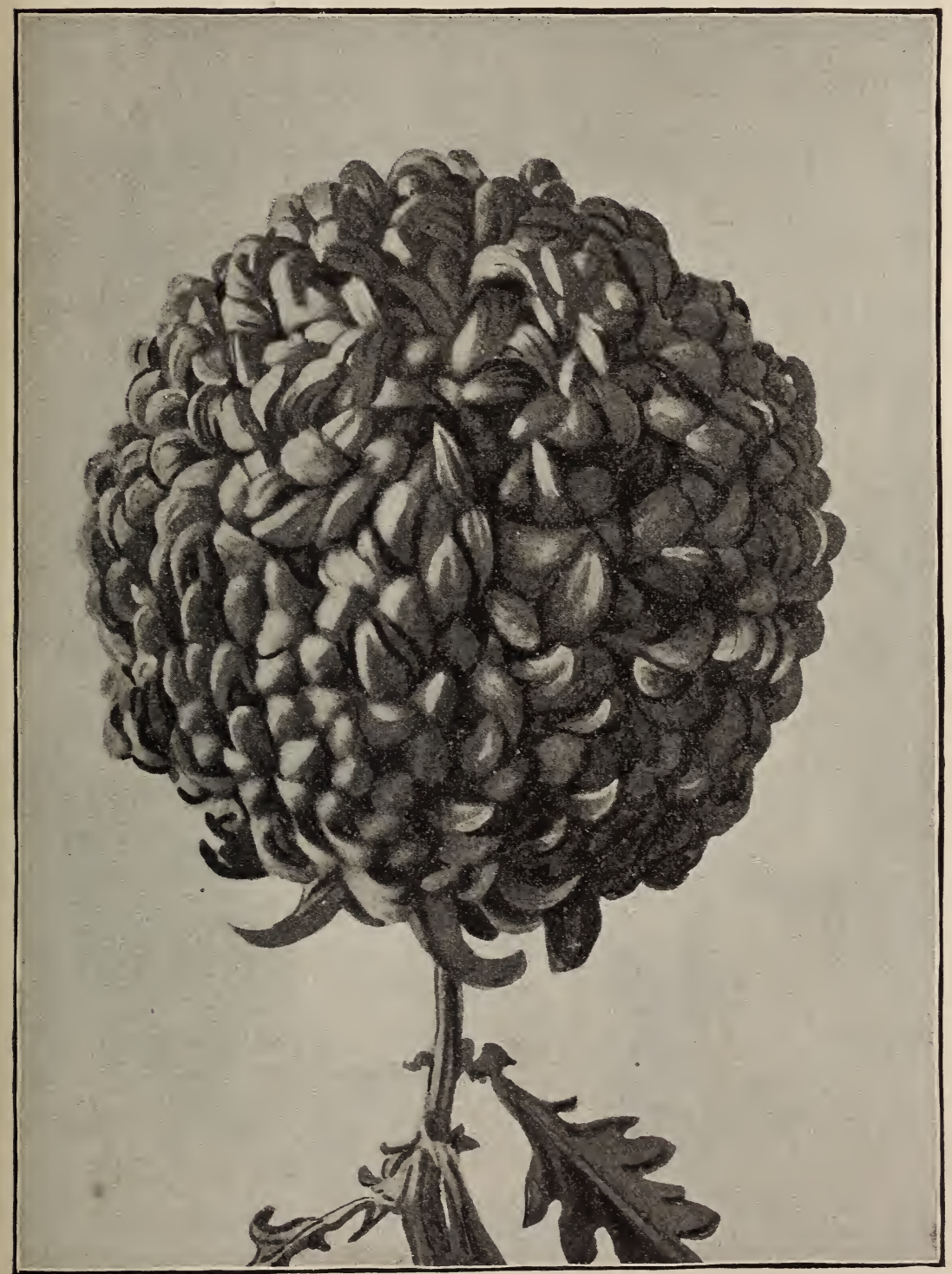

New Chrysanthemum-Mrs. H. A. Manderville. 


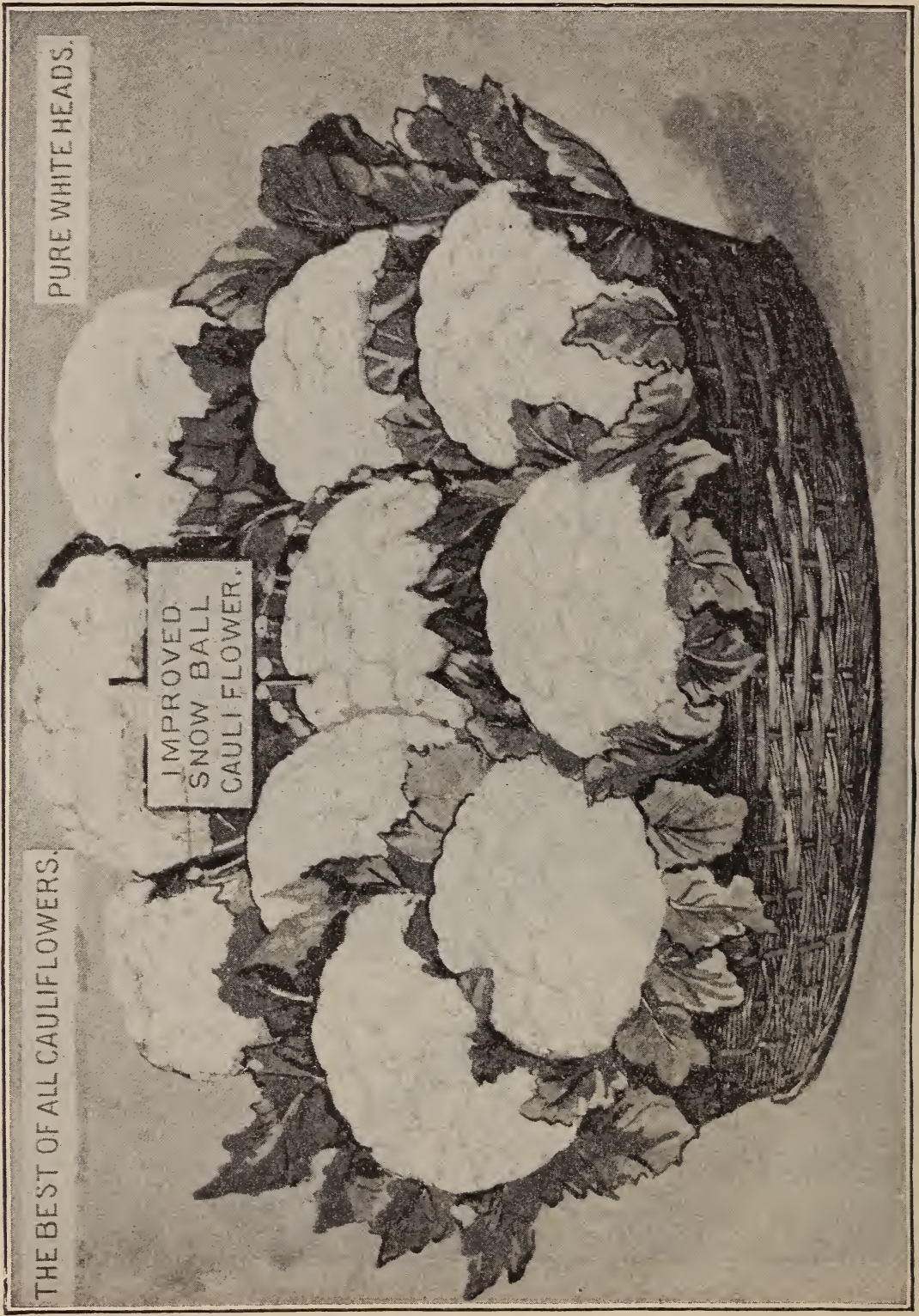

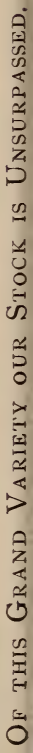




\section{General Wholesale List of Vegetable Seeds.}

We wish to call the attention, especially of market gardeners and florists to our seeds. From personal experience we know the vital importance of purity of stock and high germination. In this department purchasers may have every confidence in the goods we offer, as every article we send out will be tested both for germination and purity. We have spared ourselves no expense in the preparation of seed testing houses and trial and experimental grounds, and have secured only skilled help, many of. whom have spent a lifetime in this business.

In all cases the seeds we offer will represent the new crop of 1891 , grown by reliable men under our personal supervision.

Quarter pound furnished at pound rate, ten pound at hundred pound rate, and peck or quarter bushel at bushel rate.

When seeds are to be sent by mail, eight cents per pound for seeds sold by the pound, and fifteen cents per quart for peas and beans, must be enclosed for postage.

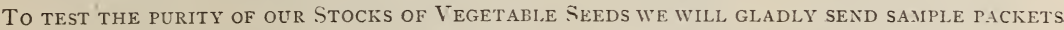
FREE UPON APPIICATION, ASKING THAT SAME IVILL BE REPORTED UPON AT MATURITY.

\section{ARTICHOKE- \\ Large Globe. . \\ Jerusalem. Strong tubers, $\$ 0$. \\ ASPARAGUS-
Palmetto..... \\ Pkt. \\ . $\$ 010$ \\ Colossal.

05 \\ ASPARAGUS ROOTS- \\ Palmetto.. \\ Pkt. \\ $\$ 0 \quad 10 \$ 0 \quad 25$ \\ 100
$25 \quad \$ 700$ \\ Colossal, two years old......... . $75 \quad 500$ \\ BEANS, Dwarf or Bush- \\ GREEN-PODDED VARIETIES.}

$1 \mathrm{~b}$.

Bus.

$\$ 300$

Earliest Red Valentine........ $20 \quad 325$

Extra Early Refugee........ $20 \quad 400$

Long Yellow Six Weeks........ 20 $20 \quad 350$

Early Mohawk.............. $20 \quad 350$

Early China............... $20 \quad 325$

Dwarf Horticultural........... $20 \quad 500$

Refugee or Thousand to One..... $20 \quad 350$

Large White Kidney........... $20 \quad 300$

White Marrow ............. $20 \quad 300$

WAX OR YELLOW-FODDED VARIETIES.

Golden-Eyed Wax........... $15 \quad 450$

Golden Wax, Improved....... $15 \quad 400$

Flageolet Wax............. $15 \quad 550$

Black Wax or Butter........... $15 \quad 425$

Wardwell's Kidney Wax..... $20 \quad 500$

White-Seeded Wax............ $25 \quad 500$

Yosemite Mammoth........... $50 \quad 1000$

BEANS, Pole or Running-

Jersey Extra Early Lima...... $40 \quad 800$

Large White Lima, Extra size.... . $30 \quad 6 \quad 50$

Dreer's Improved Lima.......... $30 \quad 6 \quad 50$

Horticultural or Speckled Cranberry $\quad 25 \quad 5 \quad 50$

Scarlet Runners.............. $30 \quad 600$

White Dutch Runners.......... $30 \quad 6 \quad 00$

Giant Wax................ 50

German Wax Pole.............. $30 \quad 700$

Early Golden Cluster Wax.... $40 \quad 800$
Bastian's Blood Turnip SU 05 oz. $\$ 010$ lb.

Dewing's Im proved

Blood Turnip, per 100

lbs., $\$ 20 \ldots \ldots \ldots \ldots \ldots$. $05 \quad 10 \quad 25$

Early Blood Turnip, per 100

lbs., $\$ 20 \ldots \ldots \ldots \ldots$

$\begin{array}{llll} & & & \\ & & \end{array}$

Egyptian Turnip. per 100 lbs., $\$ 22 \ldots \ldots \ldots \ldots \ldots .05 \quad 050.25$

Long Smooth Blood Red... $\quad 05 \quad 10 \quad 25$

Swiss Chard, Silver or Sea-

Kale Beet........... $05 \quad 10 \quad 35$

MANGEL-WURZEL and SUGAR BEET-

Oz. lb,

Champion Yellow Globe....... $\$ 0$ 05 $\$ 020$

Colossal Long Red.......... $05 \quad 20$

Golden Tankard............. $05 \quad 25$

Long Yellow............... $05 \quad 25$

Vilmorin's Improved White

Sugar Beet............................. 10

White Sugar Beet............. $05 \quad 25$

BRUSSELS SPROUTS-

Dwarf Improved Pkt. oz. lb.

Tall French........... 05 15 200

\section{CABBAGE-}

Early Blood-Red Erfurt. $\quad 05 \quad 20 \quad 200$

Early Winnigstadt....... $\quad 10 \quad 10 \quad 20 \quad 175$

Early York.............. $05 \quad 15 \quad 150$

Express............. $10 \quad 20 \quad 200$

Fottler's Improved Brunswick.................

Henderson's Early Summer (selected Long Island grown)..............

Henderson's Succession (selected Long Island grown).............

Late Flat Dutch (selected Long Island grown).....
$05 \quad 20 \quad 200$

$05 \quad 20 \quad 175$

$10 \quad 25 \quad 250$

$05220 \quad 150$ 
VEGETABLE SEEDS-Continued.

CABBAGE- Pkt,

Marblehead Mammoth Drumhead..........\$0 05

Premium Flat Dutch..... 05

Red Dutch............ 05

Selected Early Jersey Wakefield...........

American Drum'iead Savoy. 05 CARROT -

Altringham.............

Chantenay...............
Danvers Half Long, 100 lb., $\$ 40 \ldots \ldots \ldots \ldots \ldots$.

Early French Forcing.....

Early Scarlet Horn........

Half Long Red (Nantes Stump Rooted)..........

Improved Long Orange....

Oxheart or Guerande...

CAULIFLOWER-

Early Snowball (Original stock; ask for sample free packet)....1/4 lb., $\$ 10$. .

Extra Early Dwarf Erfurt...............

Nonpareil or Half Early

Paris...............

Lenormand's Short Stemmed

Algiers, Large Late.....

CELERY-

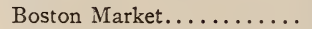

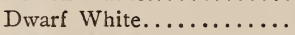

Giant Pascal...........

Giant White Solid.........

Golden Self-Blanching

(Home grown).........

Golden Dwarf...........

Half Dwarf.............

New Rose.............

White Plume..........

White Curled Solid, for

Soup Greens...........

CELERIAC or TURNIPROOTED CELERY-

Apple Shaped...........

Erfurt Giant...........

Large Smooth Prague... 10

CHICORY-

. sLarge Rooted...........

CHERVIL-

Curled.............. 0

COLLARDS-

True Georgia........... 0

CORN SALAD or

FETTICUS...

CORN, SWEET-

Adams' Extra Farly........... Per bus
Black Mexican.............. 25250

Crosby's Early................ 200

Early Cory................. 225

Early Mammoth............. 200

Early Minnesota............ 225
CORN, SWEET-continued

Per 100

Eight-Rowed.. ..............\$1 75 $\$ 225$

Henderson.................. 200 275

Hickox....................2 00 2 75

Late Mammoth............. $200 \quad 350$

Moore's Concord............... $185 \quad 175$

Old Colony.................. $185 \quad 150$

Narragansett...............2 $00 \quad 150$

Ne Plus Ultra..............2 $200 \quad 300$

Perry's Hybrid.... ........... 2 $25 \quad 225$

Potter's Excelsior (Squantum)..... $200 \quad 200$

Russell's Prolific..............2 $00 \quad 150$

Shaker's Early............... $200 \quad 150$

Stowell's Evergreen............ $190 \quad 250$

CRESS OR PEPPER GRASS-

Pkt. oz. lb.

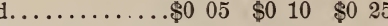

Water, True........... $10 \quad 30 \quad 300$

CUCUMBER-

Early Cluster............ $05 \quad 10 \quad 45$

Early Frame........... $05 \quad 10 \quad 45$

Early Russian........... $05 \quad 10 \quad 45$

$\begin{array}{llll}\text { Extra Early Green Pro- } & & & \\ \text { lific. } & & \end{array}$

Extra Long White Spine $05 \quad 10 \quad 40$

Improved Early White

Spine............. $05 \quad 10 \quad 40$

Green Prolific. ......... $05 \quad 10 \quad 45$

Long Green............ $05 \quad 10 \quad 50$

Short Green or Gherkin.... $05 \quad 10 \quad 45$

West India Gherkin or Burr $\quad \begin{array}{llll}10 & 20 & 1 & 00\end{array}$

ENGLISH FORCING CUCUMBERS-

Per 100 Seeds.

175

150

200

Sion House...................\$1 00

Rollison's Telegraph............... 150

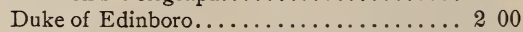

Tender and True................ 250

Lord Kenyon's Favorite............ 125

DANDELION-

Common............. \$0 $05 \$_{0} 20$ \$1 25

Large-Leaved......... $10 \quad 30 \quad 225$

EGG-PLAN T-

New York Improved.... $10 \quad 25 \quad 350$

Early Long Purple........ $10 \quad 35 \quad 150$

Black Pekin........... $10 \quad 40 \quad 300$

ENDIVE-

Green Curled........... $05 \quad 15 \quad 100$

Moss Curled................ $05 \quad 20 \quad 120$

White Curled........... $05 \quad 15 \quad 150$

Broad-Leaved Batavian Escarolle............. $05 \quad 20 \quad 130$

KALE or BORECOLESiberian, German Greens or "Sprouts"......... $05 \quad 10 \quad 50$

Dwarf Green Curled Scotch............. $05 \quad 10 \quad 60$

! Brown German Curled..... $05 \quad 10 \quad 100$ KOHL-RABI-

Early White Vienna...... $05 \quad 20 \quad 250$

Early Purple Vienna...... $05 \quad 20 \quad 250$

LEEK-

American Flag......... $05 \quad 20.175$

Musselburgh ......... $10 \quad 20 \quad 200$ 


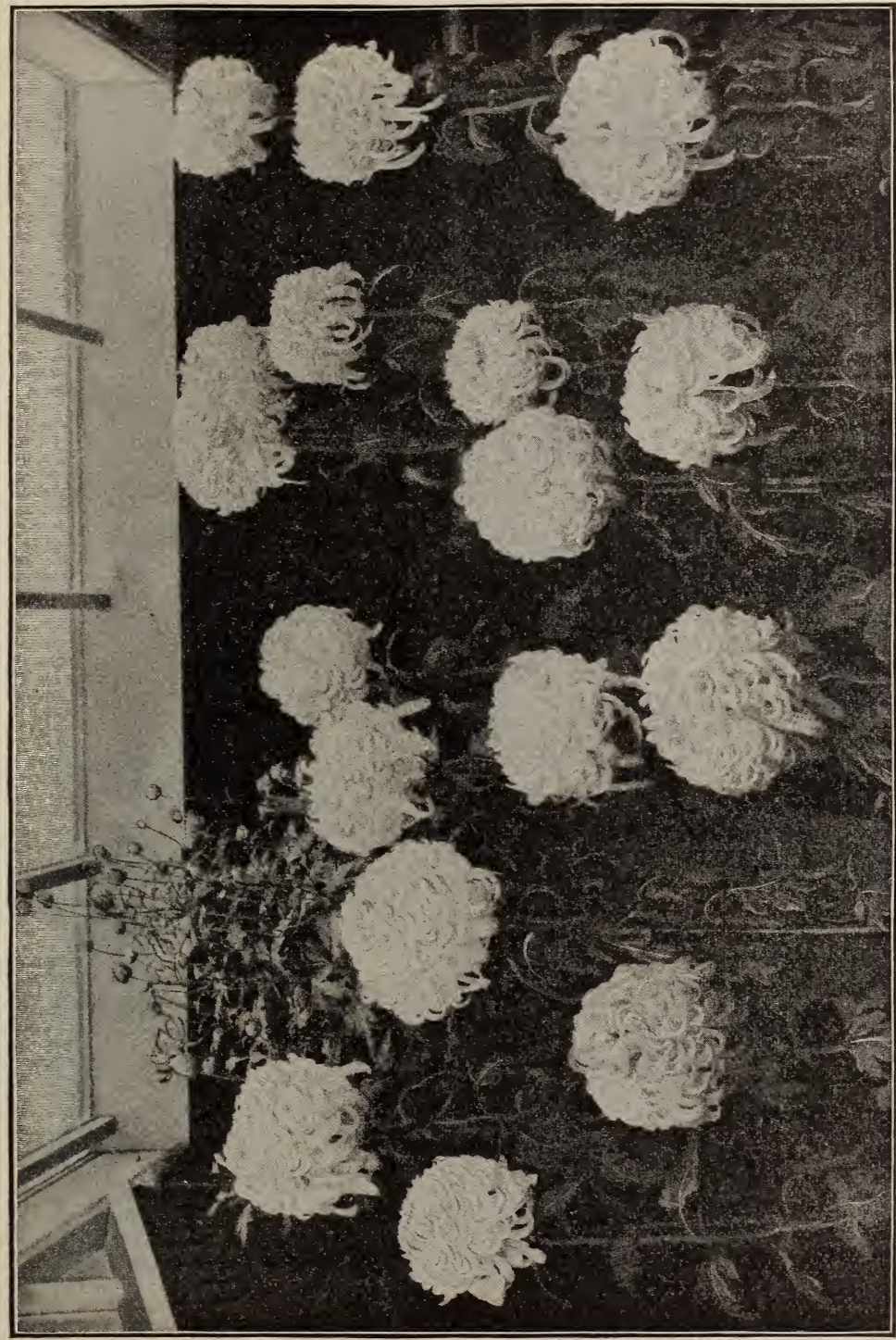

1 


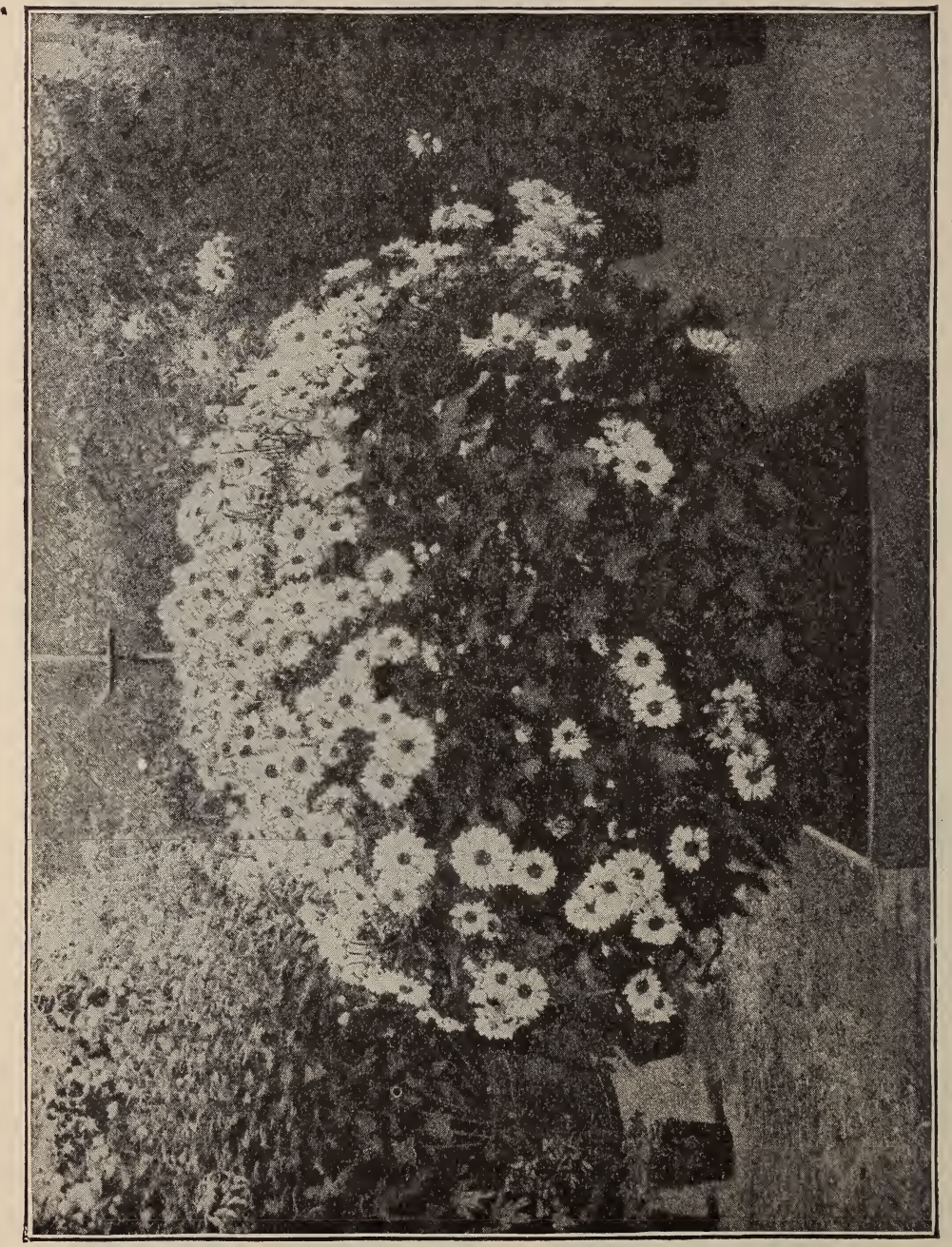

. 
VEGETABLE SEEDS-Continued.

LETTUCE-

All-the-Year-Round ....... $\$ 0$ 05 $\$ 010 \$ 0 \quad 75$

Big Boston. True ..... $05 \quad 20 \quad 125$

Black-Seeded Butter.... 050 15 65

Black-Seeded Simpson.... 05

Boston Market.......... 05

Brown Dutch.......... 05

Deacon............... 05

Drumhead or Malta...... 05

Early Curled Simpson..... 05

Hanson.............. 05

Hardy Green Winter....... 05

Large White Summer ${ }_{\mathbf{C}} \mathrm{Cab}-$ bage................

New York. True......

Paris White Cos........

Salamander............

Tennis Ball (black seeded)..

Trianon Cos...........

Yellow-Seeded Butter..

MELON, Musk-

Acme or Baltimore......

Extra Early Hackensack

Emerald Gem..........

Golden Netted Gem.......

Green Citron........... 05

Hackensack........... 05

Improved Christiana...... 05

Jenny Lind........... 05

Large Yellow Cantaloupe... 05

Montreal Market......... 05

Nuimeg.............. 05

Skillman's Netted........ 05

MELON, Water-

Black Spanish..........

Cuban Queen............

Georgia Rattlesnake or Gipsy

Green and Gold.........

Mammoth Ironclad.....

Ice Cream (White Seeded).

Kolb's Gem............

Mountain Sprout.........

Mountain Sweet.........

Phinney's Early..........

Pride of Georgia........

Ruby Gold.............

Citron (for preserving) Red

\section{MUSHROOM SPAWN-}

English .................. $\$_{0} 10$

French ....................... 30

\section{MUSTARD-}

oz. $\quad$ lb.

White London............... \$0 $05 \quad \$ 015$

Brown or Black............. 05

NASTURTIUM or INDIAN CRESSPkt. oz. 1b.

Tall. Flowers Mixed Colors $\$ 0 \quad 05 \quad \$ 0 \quad 10 \quad \$ 0 \quad 50$

Dwarf............... $05 \quad 15 \quad 75$

OKRA, or GUMBO-

White Velvet........ $05 \quad 10 \quad 50$

Dwarf Prolific........... $05 \quad 10 \quad 25$

Long Green........... $05 \quad 10 \quad 25$
ONION-

Danvers Yellow.. Round. $\$ 0,05 \quad \$ 0 \quad 10 \quad \$ 1 \quad 25$

" " Globe. $05 \quad 10 \quad 125$

Extra Early Flat Red.. $05 \quad 10 \quad 125$

Southport Red Globe... $05 \quad 15 \quad 135$

White Globe. $05 \quad 20 \quad 225$

Yellow Globe. $05 \quad 15 \quad 135$

Wethersfield Large Red. $\quad 05 \quad 10 \quad 115$

White Portugal......... $05 \quad 20 \quad 225$

Prize-Taker........... $05 \quad 20 \quad 250$.

Imported Italian Varieties.

Giant Rocca.......... $05 \quad 10 \quad 125$

" Red Italian Tripoli $05 \quad 10 \quad 125$

"White Italian

Tripoli........ $05 \quad 10 \quad 125$.

New Adriatic Barletta.. $05 \quad 20 \quad 250$

Pompeii........................... $05 \quad 15 \quad 150$

Queen.................. $05 \quad 15 \quad 150$

Bermuda Pale Red....... $05 \quad 20 \quad 150$

ONION SETS-

White ) $\ldots \ldots \ldots \ldots \ldots \$ 40$

Yellow $\}$ prices variable............ 400

Red $\int \ldots \ldots \ldots \ldots \ldots \ldots$.......... 00

Potato Onions

Top or Button Onions $\}$ Prices upon application:

Shallots

PARSNIP-

Long Smooth or Hollow

Crown............... $\$ 0$ 05 $\$ 010 \$ 0 \quad 30$

Early Round.......... $05 \quad 10 \quad 50$

PARSLEY-

Emerald............ $05 \quad 15 \quad 75$

Extra Double Curled, 100

lb., $\$ 45 \ldots \ldots \ldots \ldots \ldots .05 \quad 0 . \ldots \ldots$

Hamburg, or Turnip-Rooted $05 \quad 10 \quad 60$

PEAS, Extra Early and Second Early-

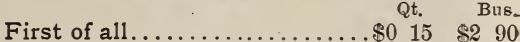

American Wonder........... $20 \quad 400$

Extra Early Premium Gem. ... $\quad 20 \quad 300$

Horsford's Market Garden...... $20 \quad 315$

Improved Daniel O'Rourke. (Selected Stock).............. $15 \quad 260$

McLean's Advancér.. ........ $20 \quad 20 \quad 275$

McLean's Little Gem........... $20 \quad 30 \overline{0}$

Maud S. Extra Select Strain of Extra Early............... $20 \quad 290$

Philadelphia Extra Early.......... $15 \quad 250$

Tom Thumb............... $20 \quad 400$

PEAS, for General Crop-

Abundance................ $20 \quad 300$

Black-Eyed Marrowfat. (Hand picked)..................... $10 \quad 175$

Champion of England.......... $20 \quad 260$

Culverwell's Telegraph........ $20 \quad 400$

Everbearing. ............ $20 \quad 310$

Pride of the Market......... $20 \quad 425$

Stratagem.............. $20 \quad 500$

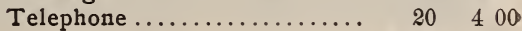

White Marrowfat. ........... $15 \quad 175$

Yorkshire Hero.............. $20 \quad 260$

Canada Field, white for soiling..... 140 


\begin{tabular}{|c|c|c|c|}
\hline PEPPER- & Pkt. & oz. & lb. \\
\hline Child's Celestial... &.$\$ 005$ & $\$ 040$ & $\$ 200$ \\
\hline Sweet Spanish........ & 05 & 25 & 150 \\
\hline Large Bell, or Bull Nose... & 05 & 25 & 150 \\
\hline $\begin{array}{l}\text { Sweet Mountain or Mam } \\
\text { moth } \ldots \ldots \ldots \ldots \ldots \ldots\end{array}$ & 05 & 25 & 150 \\
\hline Ruby King............ & 05 & 35 & 160 \\
\hline Monstrous or grossium.... & 05 & 30 & 300 \\
\hline Long Red Cayenne........ & 05 & 25 & 125 \\
\hline 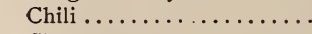 & 05 & 25 & 175 \\
\hline Cherry $\ldots \ldots \ldots \ldots \ldots$ & & & 175 \\
\hline
\end{tabular}

POTATOES-We are in position to furnish all the leading kinds, and would be glad to quote prices for any quantity and kind upon application.

\section{PUMPKIN-}

Large Cheese........... $\$ 0 \quad 05 \quad \$ 0 \quad 10 \quad \$ 0 \quad 50$

Large Tours, or Mammoth.

Connecticut Field, Bush, $\$ 3$.

$20 \quad 50$

RHUBARB-

Linnæus............. 05

Victoria.............. 05

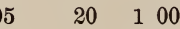

RADISH-

Beckert's Chartier........ $05 \quad 10 \quad 40$

California Mammoth White

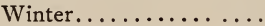

Early Scarlet Globe........

Early White Turnip........

Early Round Dark Red.

Per 100 lbs., \$25. (10

lbs. at $100 \mathrm{lb}$. rates).....

Early Scarlet Turnip.

Per 100 lbs., $\$ 22$. (10 lbs.

at $100 \mathrm{lb}$. rates).........

French Breakfast.........

Giant White Stuttgart. ....

Long Scarlet Short-Top....

Long Black Spanish.......

Long White Spanish ......

Long White Vienna or Lady

Finger.............

Olive-shaped Scarlet.......

Red Forcing Turnip.....

Rose China Winter.....

White-tipped Scarlet

Turnip. Per 100 lbs. $\$ 30$.

(10 lbs. at $100 \mathrm{lb}$. rates). .

White Summer Turnip.....

White Olive-Shaped. ......

White Strasburgh.........

Yellow or golden Summer

Turnip............ $05 \quad 10$

SALSIFY or OYSTER PLANT-

Long White........... $05 \quad 10$

Improved Mammoth Sand-

wich Island.

$05 \quad 15,80$

SCORZONERA orBLACK OYSTER PLANT...

SEA-KALE.......... 10

$\begin{array}{llll}05 & 15 & 1 & 00\end{array}$

SORREL-

Broad-Leaved.........

\section{SPINACH-}

Bloomsdale or Norfolk

Savoy-Leaved.........

Round-Leaved. $\$ 11$ per 100

lbs..................

Long Standing. $\$ 12$ per 100 lbs...............

Prickly. $\$ 14$ per 100 lbs...

Large Round-Leaf Viroflay. $\$ 12$ per 100 lbs........

Thick-Leaved. $\$ 12$ per

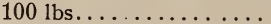

New Zealand...........

oz. $\quad$ lb.

$\$ 0 \quad 05 \$ 0 \quad 20$

05

15

05

15

05

20

05

15

05

15

\section{SQUASH-}

Boston Marrow. Per 100, $\$ 3000 \ldots \ldots \ldots \ldots \ldots$

Bush Summer Crook-Neck..

Early Prolific Marrow...

Essex Hybrid...........

Fordhook.............

Hubbard. ..............

Mammoth Chili..........

Perfect Gem............

Pine Apple............

Sibley..............

White Bush Scalloped..

Yellow Bush Scalloped..

TOBACCO SEED-

Gold Leaf............. $05 \quad 25 \quad 300$

Hester................ $05 \quad 25 \quad 275$

Florida.............. $05 \quad 25 \quad 300$

Sumatra.............. $05 \quad 25 \quad 350$

Primus. ............ $05 \quad 25 \quad 350$

Connecticut Seed-Leaf..... $05 \quad 25 \quad 250$

Imported Havana......... $05 \quad 50 \quad 350$

Virginia......................... $05 \quad 05 \quad 25 \quad 300$

Kentucky.............. $05 \quad 25 \quad 300$

Maryland.............. $05 \quad 25 \quad 250$

TOMATO-

Acme................. $05 \quad 20 \quad 125$

Early Ruby. (True)... $10 \quad 10 \quad 30 \quad 175$

Essex Hybrid........... $05 \quad 20 \quad 125$

Golden Queen........... $05 \quad 15 \quad 175$

Hathaway's Excelsior...... $05 \quad 15 \quad 150$

Livingston's Favorite...... $05 \quad 15 \quad 125$

Livingston's Beauty.... $05 \quad 15 \quad 150$

Mikado............... $05 \quad 20 \quad 175$

New Dwarf Champion... $\quad 05 \quad 15 \quad 200$

Paragon............... $05 \quad 15 \quad 125$

Perfection............... $05 \quad 15 \quad 125$

Queen............... $05 \quad 15 \quad 125$

Table Queen. (True)..... $10 \quad 15 \quad 175$

Trophy, Extra Selected. $\quad 05 \quad 15 \quad 175$

Volunteer.............. $05 \quad 15 \quad 150$

Red Pear-shaped........ $10 \quad 20 \quad 200$

Yellow Plum............. $10 \quad 20 \quad 200$

Red Cherry............. $10 \quad 20 \quad 300$

Greengage.............. $10 \quad 20 \quad 200$

Strawberry or Winter Cherry $\quad \begin{array}{llll}10 & 20 & 300\end{array}$

Peach................ $10 \quad 20 \quad 160$

TURNIP-

Flat Dutch (Early)....... 20

Golden Ball............ 


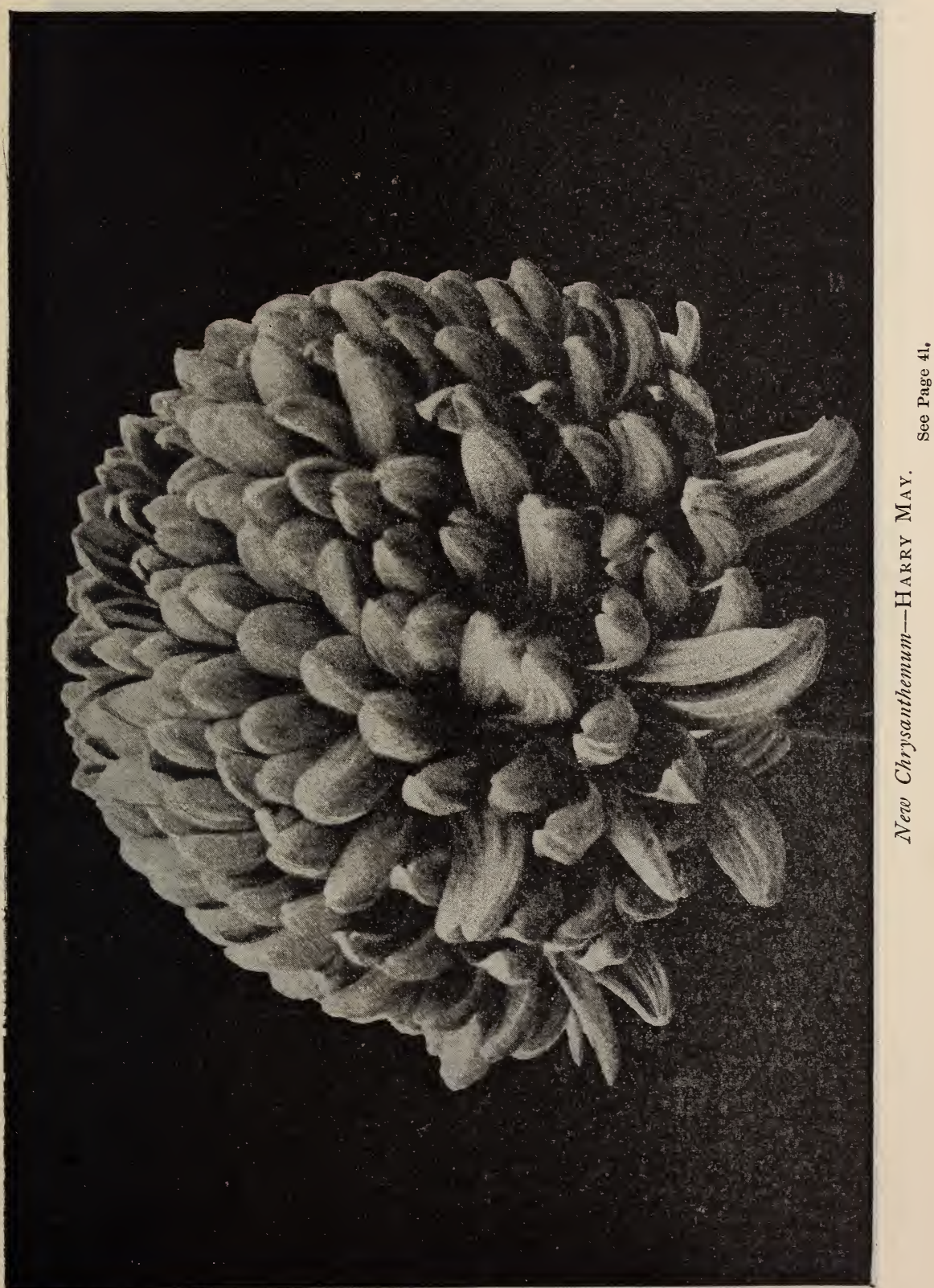




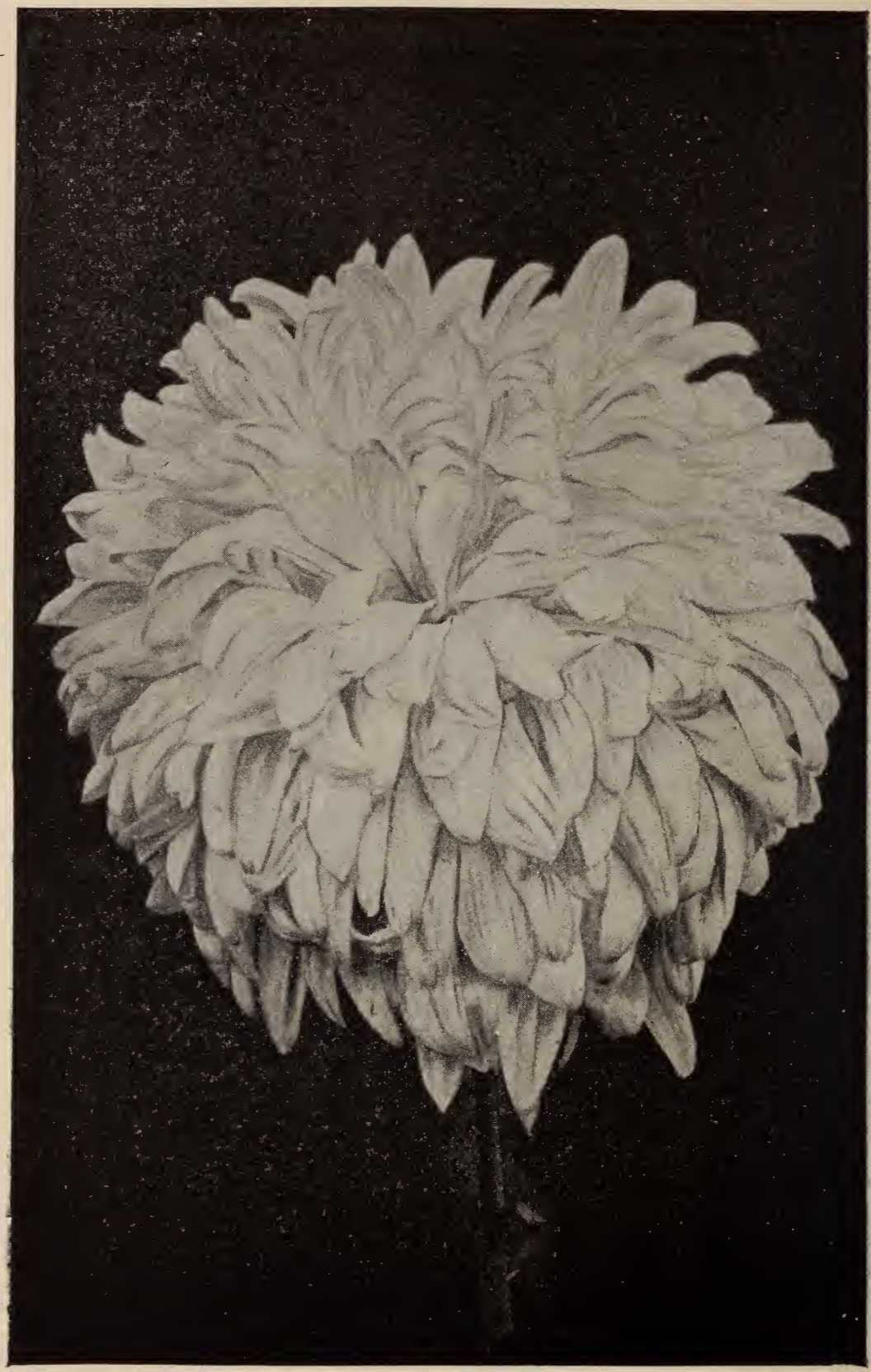

Neze Chrysanthemum-AdA MCVICKAR. 
VEGETABLE SEEDS-Continued.

TURNIP-

Milan Extra Early......

Purple-Top White Globe...

Red-Top Strap-Leaf....

White Egg............

White Strap-Leaf (Early)...

Yellow Globe... .......

Yellow Stone............

RUTA BAGA-

c. Purple-Top I mproved

American ............

Skirving's Purple Top......

Large White French.......

SWEET POT and MEDICINAL HERBS-

Anise.
Pkt. oz. Ib. $\$ 0 \quad 10 \$ 0 \quad 50$

10 . 20

$10 \quad 20$

$10 \quad 22$

$10 \quad 25$

$10 \quad 23$

$10 \quad 25$

$10 \quad 25$

1020

$10 \quad 35$

$05 \quad 10$
HERBS-continued

Balm ................ $\$ 0 \quad 05 \$ 030 \$ 300$

Basil, Sweet.......... $05 \quad 25 \quad 125$

Bene................... $05 \quad 10 \quad 100$

Dill............... $05 \quad 10 \quad 50$

Fennel, sweet.......... $05 \quad 10 \quad 75$

Horehound............. $05 \quad 40 \quad 250$

Hyssop............... $05 \quad 20 \quad 250$

Lavender.............. $05 \quad 20 \quad 100$

Majoram, Sweet....... $05 \quad 20 \quad 150$

Rosemary............. $05 \quad 30 \quad 400$

Rue............................... $05 \quad 050$

Sage ............... $05 \quad 20 \quad 175$

Savory Summer.......... $05 \quad 20 \quad 100$

Thyme, Broad-leaved Eng.

lish ............. $05 \quad 25 \quad 275$

Tansy................ $05 \quad 30 \quad 250$

\section{Sundry Agricultural Seeds.}

GRASSES-

Finest Mixed Lawn Grass.....

English Rye Grass..........\$0 05

Italian Rye Grass ........... 10

Red Top Grass............. 05

" " Absolutely clean. 10

Kentucky Blue Grass. Fancy; extra clean.................. 20

Orchard Grass................ 15

Timothy or Herd Grass. $45 \mathrm{lbs}$. to bushel. Market price (present).

\section{CLOVER-}

Alsike......................... $\$ 022$

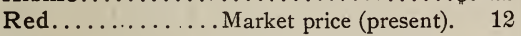

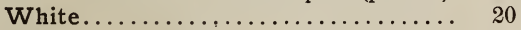

Alfalfa....................... 16

Sainfoin....................... 15

Scarlet..................... 20

Japan....................... 50

\section{SORGHUM-}

Broom Corn, Evergreen ........... 10

Doura, Rural Branching............. 15

"Yellow Branching........... 15

Kaffir Corn..................... 15

Sugar Cane, Early Amber. Per bu., \$3. 10

Early Orange....... " 3.10

MILLET-

Per bu.

Hungarian Grass. 48 lbs. to bushel... .\$2 00

German or Golden... Market price, about 200
GRAINS, ETC.- Per bu.

Rye, Excelsior Winter...........\$1 50

Wheat. Prices on application....... about 200

Buckwheat, Silver Hull........... 100

New Japan........... 150

Wild Rice.............per lb., 25 cts. .

Vetches, or Tares.............. 350

Russian Sunflower. 25 lbs. to bushel.. 200

Cotton Seed, Sea Island..per lb., $10 \mathrm{cts}$.

Osage Orange......... " 20 cts.

\section{BIRD SEED-}

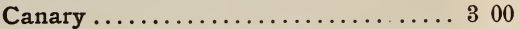

Hemp...................... 300

Rape.................... 250

Maw............... 20 cts per lb.

FRUIT SEEDS- Peroz. lb. bu.

Apple............. $\$ 030 \quad \$ 800$

Cherry, Mahaleb........

Currant, Red, White and

Black............. $30 \quad 250$

Gooseberry, Red, Yellow

and Green.......... 100

Peach Pits............

275

Pear................. $20 \quad 200$

Quince............... $20 \quad 200$

Raspberry........... $40 \quad 300$

Strawberry, Large and

Red Alpine......... 75 


\section{Plant Novelties.}

'The following are the most desirable new plants, recommended to the Florists' trade. They are plants that no progressive Florist can afford to be without, while a grand lot of new Chrysanthemums will be found under another heading.

PTERIS VICTORIAE. One of the grandest Ferns ever introduced, originally discovered by $I$. Forsterman. The foliage is neatly divided, the sterile fronds being much broader than the fertile ones. The middle of the frond is beautifully variegated with silvery white, giving the plant a most unique appearance.

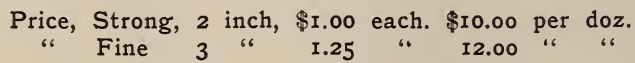

NEW GENISTAS. The following set was raised from seed by Mr. JAs. DEAN, and as everybody knows, raised quite a sensation when exhibited at the Lenox Lyceum Exhibition. Genistas have become such popular flowers for Easter decoration that they are indispensable to every Florist. The hybrids as described below are improvements upon the common type, having finer foliage, and a good many having the desirable drooping habit; others again have new colors in flower, and altogether, it is a set of plants that nobody can afford to be without. Plants will be ready March 1st.

50c. each; $\$ 5.00$ per dozen for any of the varieties, or $\$ 5.00$ for the set of I2.

No. 1. El Dorado-A most distinct variety, drooping habit. The flower spikes are large and of a bright yellow, and the plant remains in flower longer than any of the other Genistas.

No. 2. Gold-Beautiful foliage ; flower of good size, deep golden yellow color ; habit good, 'and it will make an excellent variety.

No. 3. California-Very fine Genista, with good, distinct foliage ; flower deep yellow, of good size and substance. Genista.

No. 4. Lemon-A pale lemon or yellow color, which is a most distinct shade of color in the

No. 5. Canary-Flower light shade of yellow; foliage distinctly cut and of a new type.

No. 6. Oriole-Beautiful plant with dark golden yellow flowers and good foliage.

No. 7. Arizona-Very strong grower, fine form and foliage ; good yellow flower.

No. 8. Primrose-Foliage very distinct and fine cut ; flowers pale yellow.

No. 9. Golden Gate-Fine habit, distinct foliage of a dark green color ; flowers bright yellow. A good plant.

No. 10. Amber-Foliage distinct; flowers very light lemon color.

No. 11. Sunshine-Fine robust grower, good foliage; flowers large and of a most beautifu? bright yellow color.

No. 12. Citron-Very distinct foliage. Plant produces fine spikes of golden yellow flowers.

\section{Florists' Set of New Chrysanthemums.}

We have selected the following varieties, embracing the very cream of the different sorts sent out by us this year of our own seedlings, as being superior to all others for commercial purposes. When we say superior, we mean it. What Florist wants to spend his time and money cultivating inferior sorts which command a low price, when he can just as well grow a superior article at a greater profit? Every year brings great improvements in the habit, character and quality of this favorite Fall Flower, and the success attained by us, taking every fırst premium for pot plants offered at the Madison Square Chrysanthemum Show, Nov. $2 \mathrm{~d}$ to 9 th, proves this fact. This we have accomplished with our new Seedlings, which has thus established their superiority over existing kinds. 


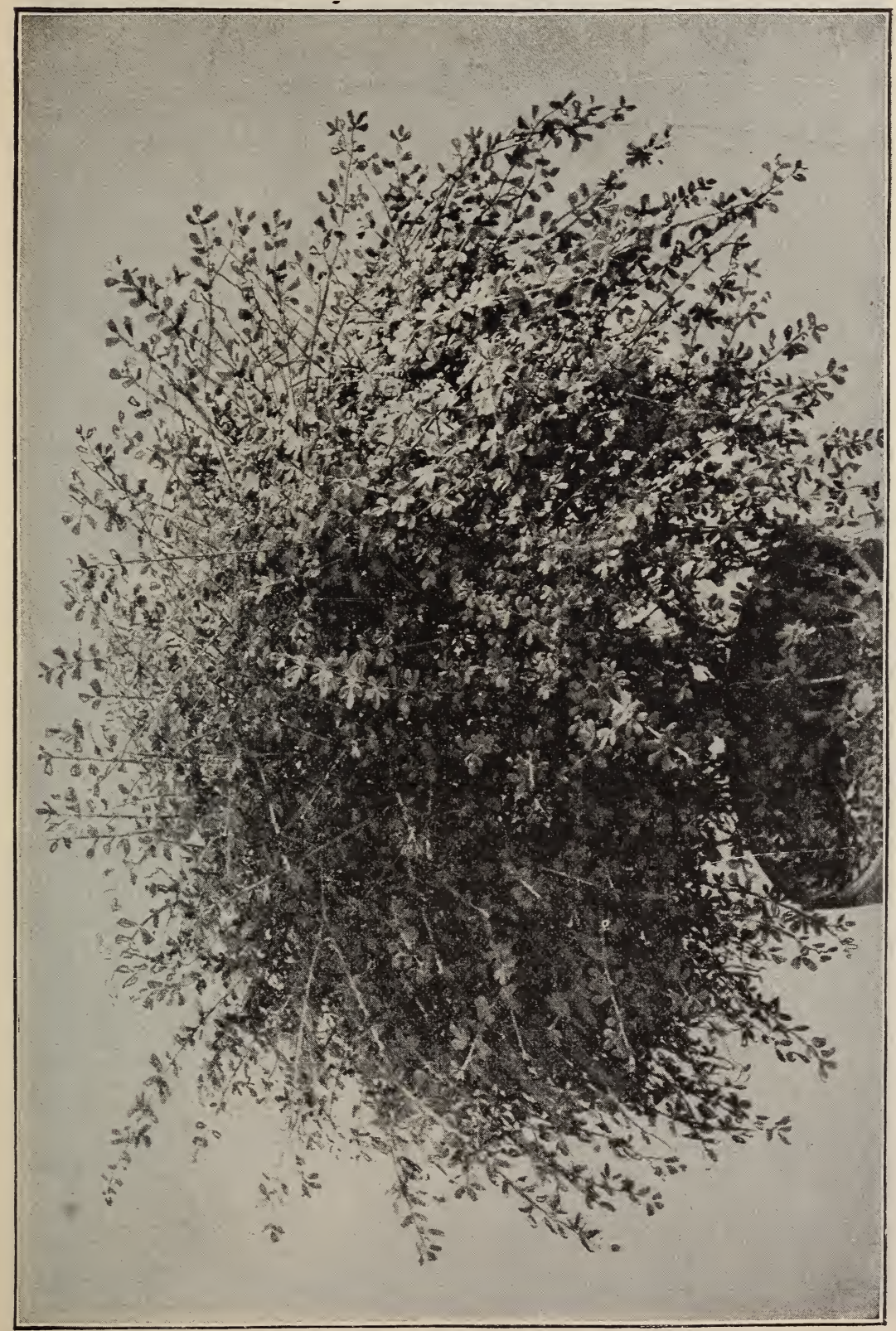

范 


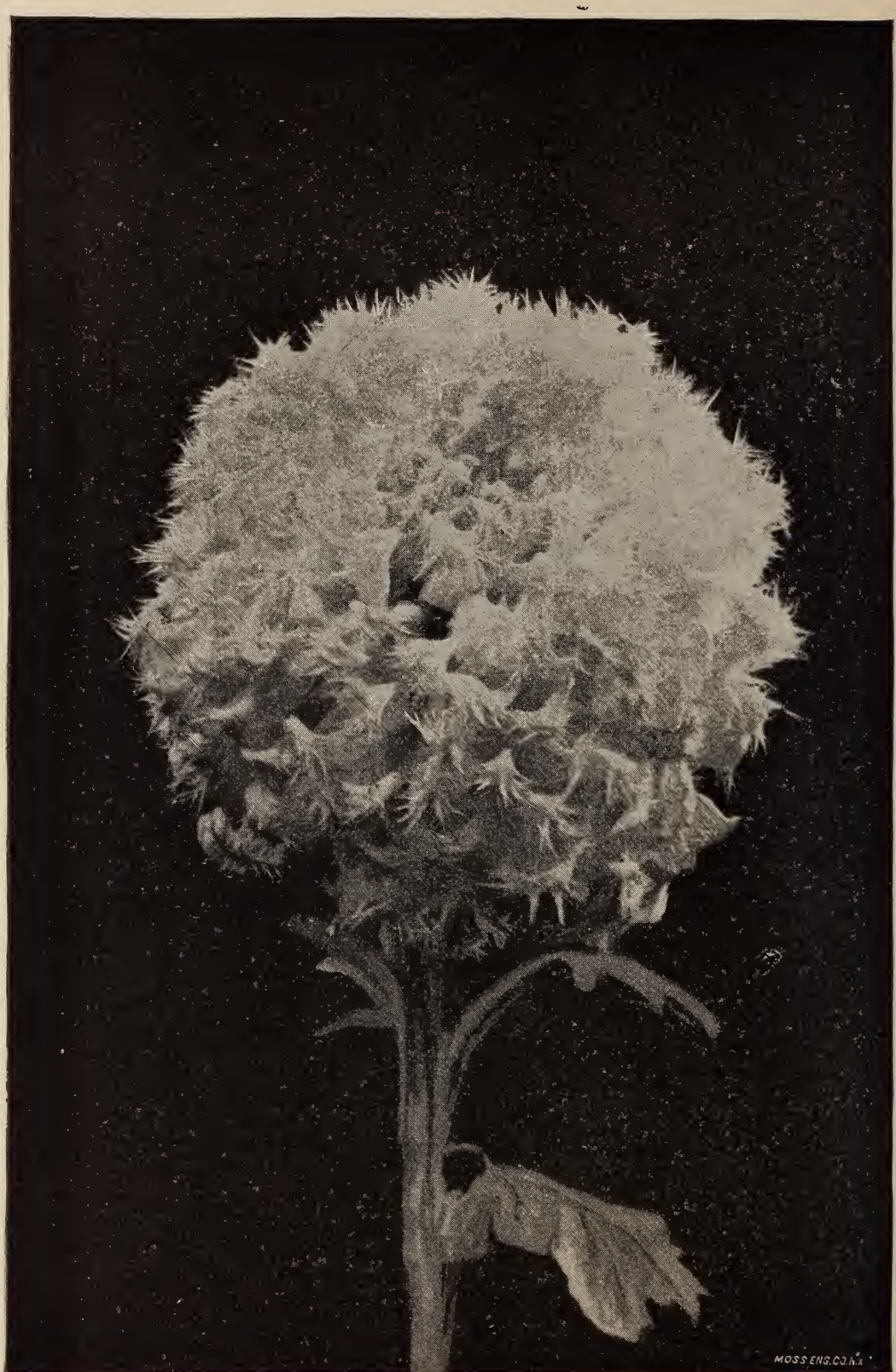

Nere Chry anthe num-Miss Invif. MaNida. See Page 41. 


\section{New Chrysanthemums.}

The large size of the flowers, their strong habit of growth and excellent shipping qualities, recommend them to all, and to those growing flowers for profit we would say, discard all the old worthless sorts and book up with the prize winners.

Ada MeVickar-A grand variety of strong habit, producing immense creamy white flowers with broad, thick, reflexed petals. One of the best for specimen blooms, bush plants or standards.

Col. H. M. Boies-Vigorous habit. Flowers large rosy pink, with twisted petals veined with a lighter shade; full center. A grand exhibition variety.

Dr. H. A. Mandeville-Flowers large, full double, bright chrome yellow; petals very long.

Francis Tarbox-Large flowered and full center; petals broad, convex silvery pink with a narrow line of pale lilac at the extreme edge. A grand early flowering variety.

George Savage-Flowers very large, pure white, with broad, strongly incurved petals, making the flower almost hemispherical and very solid. A grand variety of vigorous free flowering habit, and useful for cut blooms for exhibition.

Harry May-Flower very large and deep, full double, forming when well opened a massive sphere, color deep old gold, with occasional veins of red ; petals very broad and thick, spoonshaped. Foliage very luxuriant, thick and leathery, deep green; quite distinct among chrysanthemums. One of the most vigorous growing varieties in cultivation, and unsurpassed for growing, either as a standard, as a bush plant or for cut blooms for exhibition.

Miss Annie Manda-Flower very high and compact, perfectly double, incurved, of the purest white. The numerous petals are well furnished with long glandular hair-like outgrowths, giving the flower a unique appearance, far surpassing the celebrated variety, "Mrs. Alpheus Hardy." The plant is of strong and vigorous habit, carrying the flower erect on a stout stem. Another recommendation is that the flower is sweet scented.

Mr. A. G. Ramsay-Flowers of medium size, full center; petals broad, upper surface of the expanded limb deep Indian red, yellowish at the tip, under surface yellowish, veined with lines of red. A grand and striking variety.

Miss Bertha N. Robison-Flowers rose pink with red centre, very early, of large size and good substance. One of the earliest and best varieties for exhibition purposes either as pot plants or cut blooms.

Mr. D. S. Brown-Flower medium size, semidouble, of a clear canary-yellow color when first opening, but changing to cream color as the petals expand. A distinct color.
Mrs. E. D. Adams-Flower very large, petals of medium width, very long, twisted; the outer ones swirled, as if the flower had been turned swiftly on its stem, color pure white. One of the best and most distinct white chrysanthemums in cultivation. We had specimens measuring eighteen inches over the flower, from tip to tip of petals. Makes a grand bush plant.

Mrs. Herbert Leon-Flowers very large, full double, pink with broad reflexed petals. A very fine early variety.

Mrs. H. A. Mandeville-Flowers large, perfectly spherical, with broad, incurved petals of a terra cotta yellow. Plant of vigorous habit and'good for exhibition purposes.

Mr. Hicks Arnold-A very strong and floriferous variety, bearing large, full double flowers of an old gold color, lighting up wonderfully by artificial light. When fully open the flower is almost spherical. The freest growing variety known, and one which may be grown with success for almost any purpose.

Mrs. J. Hood Wright-Flowers large full double, of the purest white, with reflexed twisted petals. A strong grower and one of the best early varieties in cultivation.

Mrs. John Eyerman-Flower semi-globular, full double, petals decidedly spoon-shaped, the lower half being tubular, while the limb is broadly expanded; upper surface rose pink, lower pale lilac. A grand variety for exhibition purposes.

Mrs. E. D. Church-One of the earliest varieties in cultivation; flowers full double, of medium size, borne on stout stems and lasting long in perfection. Color pink in various shades.

Miss M. Colgate-Flowers perfectly hemispherical, compact, with a full center, pure white; petals, broad incurved. A grand pure white variety, a strong grower and free bloomer. Good for all exhibition purposes.

Mrs. R. Benner-Flowers medium size, of a deep lilac pink color with a red center. A very striking late variety.

Mrs. T. F. Mercer-Flowers large, blush white, petals broad, incurved, the inner regularly imbricated, the outer more spreading. One of the best varieties for exhibition purposes, and valuable on account of its late flowering.

Mrs. W. S. Kimball-Flowers very large, full double, pale blush or creamy white with a yellowish center ; petals very broad, reflexed. One of the finest varieties for exhibition purposes 


\section{NEW CHRYSANTHEMCMS-Continued.}

Mrs. M. F. Gallagher-Flowers large, semiglobular, with full center ; petals deep maroon, crimson above, paler below. One of the very best dark varieties.

Newark-Large pure white flower, with very broad and thick round petals. The finest single white variety in cultivation.
W. A. Manda-This is the new golden yellow hairy chrysanthemum. Flowers very large, of a clear golden yellow. The plant is vigorous in growth, and the flower is borne upright on a stout stem. This is the grandest introduction from Japan made during the past year, and no collection can afford to be without this variety.

Fine strong plants ready March 1st,

Price, 75 cents each; $\$ 8$ oo per dozen; $\$ 50$ oo per hundred.

The collection of 25 Prize varieties, one of each for $\$ 16$.

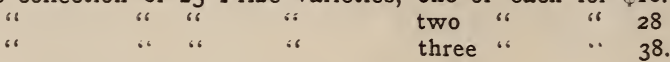

\section{GE NERAL LIST}

\section{-OF- \\ GHRYSANTHETMUTMS. \\ SUITABLE FOR FLORISTS.}
(J) Japanese.
(C) Chinese.
(A) Anemone.

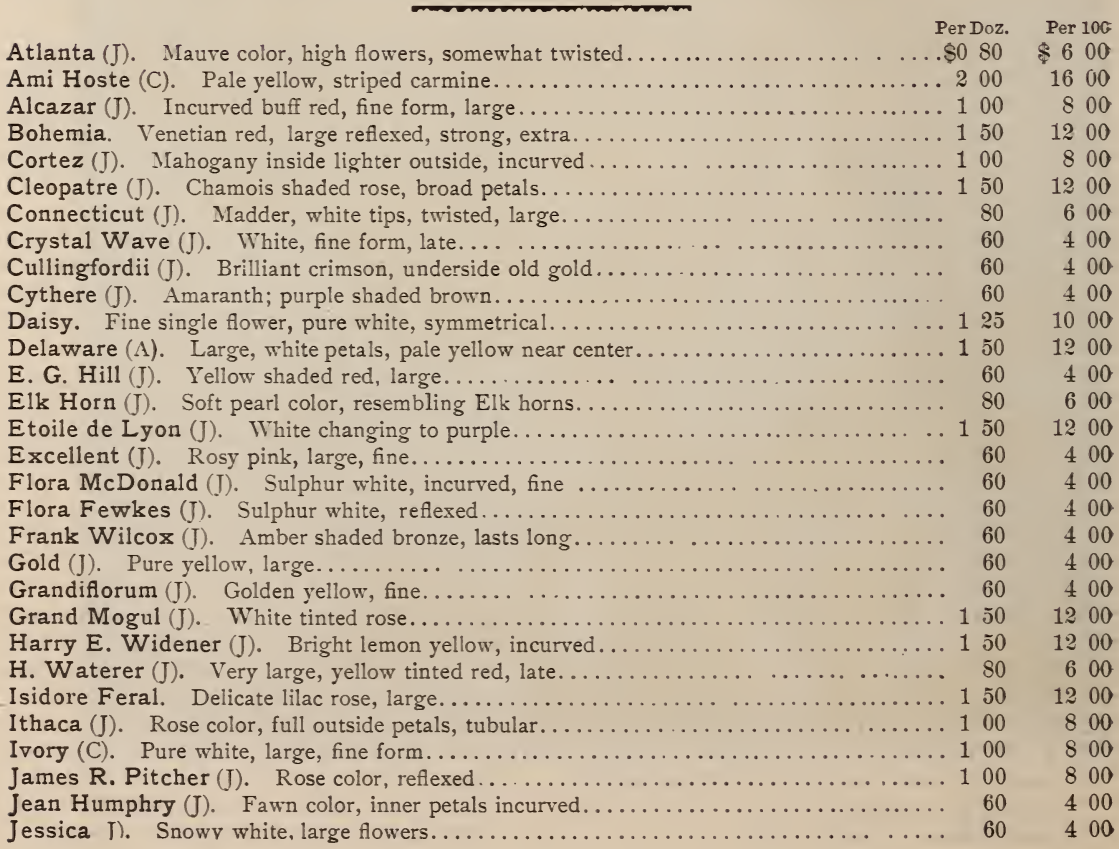




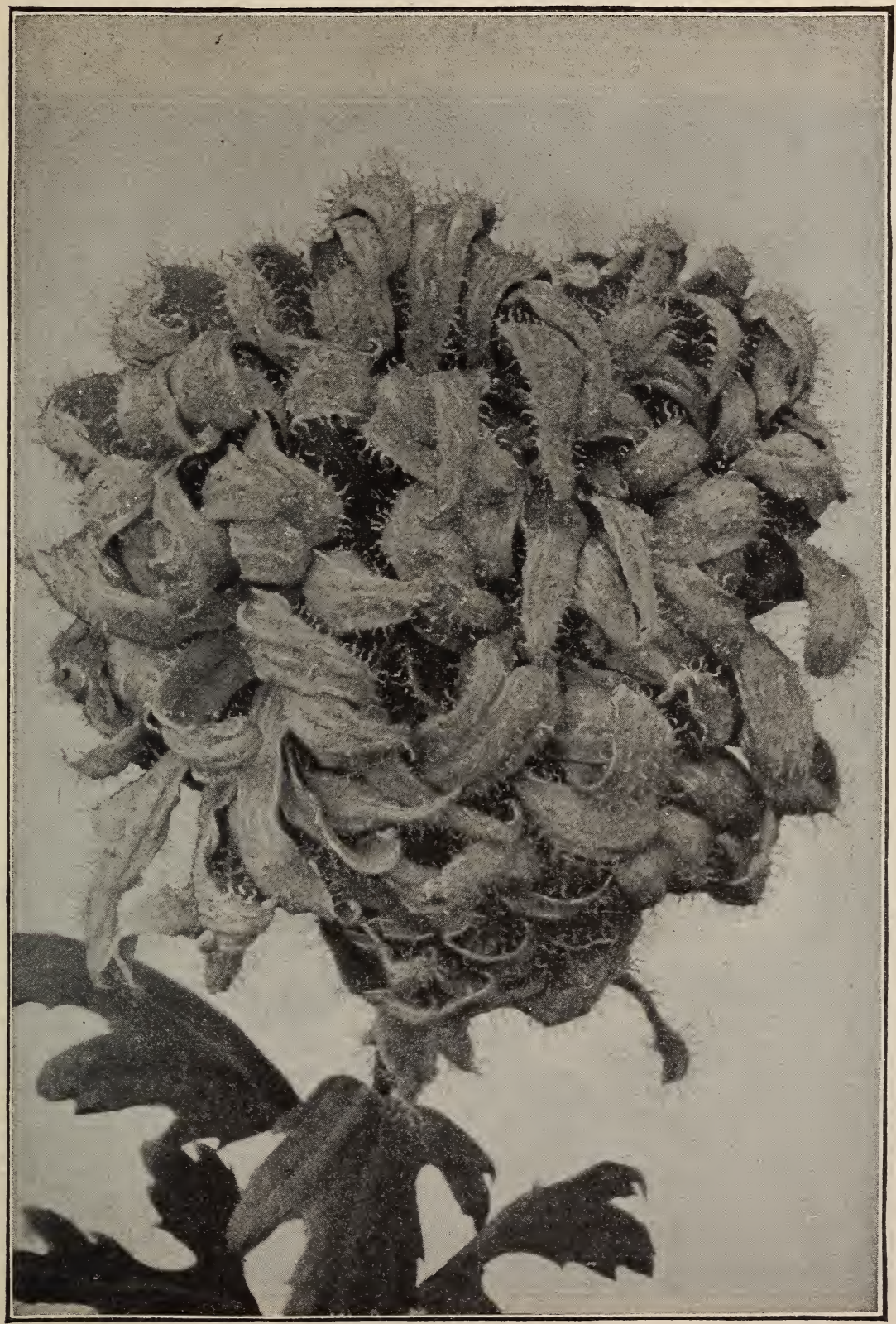

Nene Gollen Yellowe Hairy Chrysanthemum-W. A. Manda.

See Page 42. 


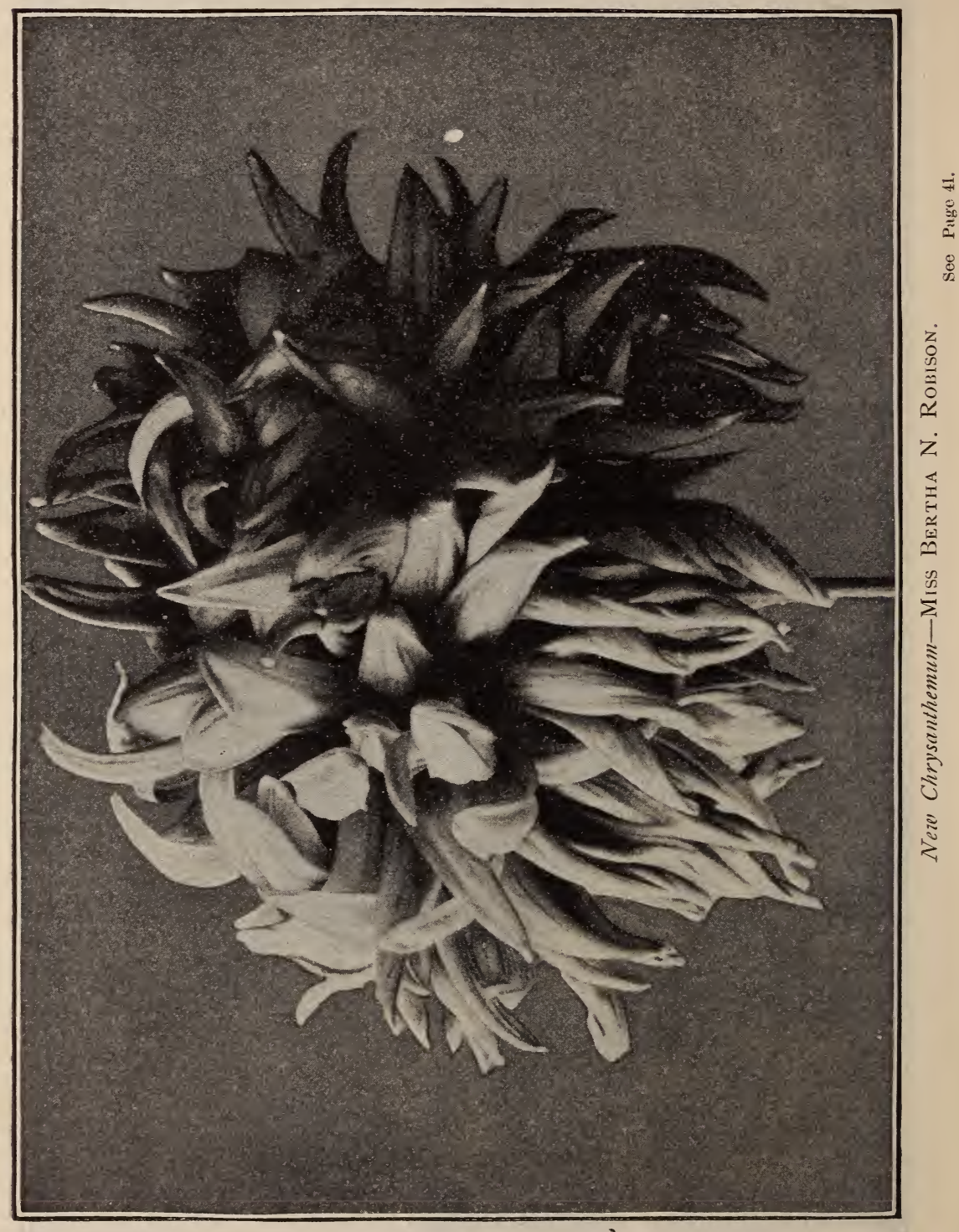




\section{CHRYSANTHEMUMS-Continued.}

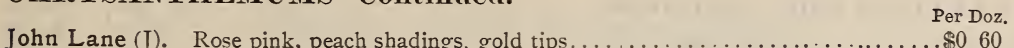

John Dyer (J) Chrome yellow striped red. gold tips........................ 60

Joe Collins (J). Coppery bronze..................................... 80

John Firth (C). Light pink, incurved, good............................ 80

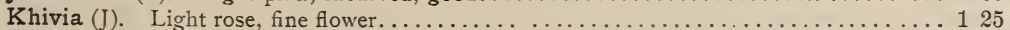

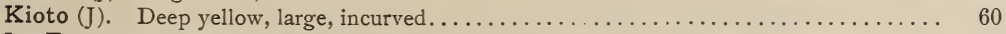

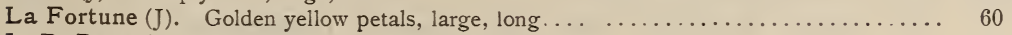

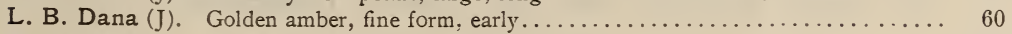

L. Canning $(J)$. White, very free, fine, double........................... 60

Le Cte. Foncher de Cariel (I). Violet, fine form.................... 60

Lilian B. Bird (J). Shrimp pink, tubular petals... . . . . . . . . . . . . . . . . . 60

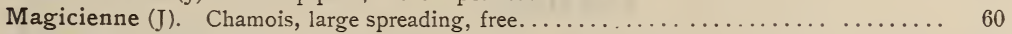

Marvel (J). White, large maroon spots............................. 60

M. Ulrich Brunner. Crimson maroon, reflexed .... ...................... 150

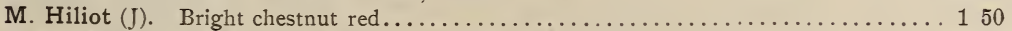

Mad. Alfred Carriere (J). Milky white, broad petals, globular................... 125

Mad. Darriar (C). Pink, large flower.................................. 150

Mad, C. Audiguier (J). Deep mauve, very large....................... 60

Mad. Bacco (J). Soft rose........................................ 60

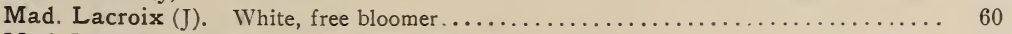

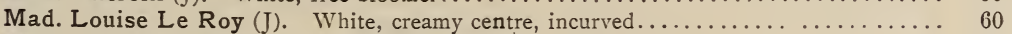

Mad. L. Langlois $(J)$. White tinted rose................................. 60

M'd'lle Camille Richard (C). Light rose, old gold center................... 150

Mermaid (J). Bright pink, fine form............................ 150

Michigan. Rich magenta, very floriferous............................ 100

Miss L. Cartledge (J). Dark rose, very fine......................... 80

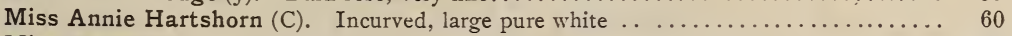

Miss Meredith $(\mathrm{J}) . \quad$ Fine pink, incurved................................... 60

Mont Blanc (J). Pure white.......................................... 60

Mrs. C. Vanderbilt (J). Very large, rose madder....................... 60

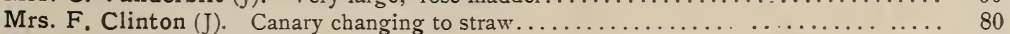

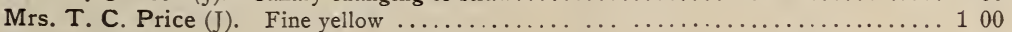

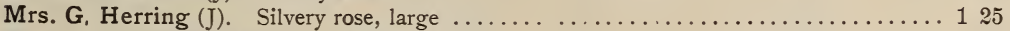

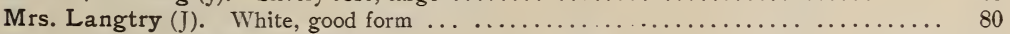

Mrs. D. D. L. Farson (J). Rose color, fine form.......................... 80

Mrs. Hicks Arnold (J). Beautiful soft rose, the finest early variety.............. 80

Mrs. Grace Hill (J). Delicate blush, incurved, grand variety................. 100

Mrs. F. Thompson (J). Silvery pink, incurved........................ 60

Mrs. Jesse Barr $(J)$. White, incurved, fine.......................... 60

Mrs. De Witt Smith (J). Soft rose changing to white.................... 60

Mrs. Libbie Allan (J). Large yellow, incurved........................ 150

Mrs. N. G. Thomas (J). Creamy white, large, incurved.................... 60

Mrs. G. B. Topham. White tinted lavender, semi-double... .............. 60

Pelican $(\mathrm{J})$. White, large flowers, broad curled florets....................... 60

Pres. Hyde (J). Rich yellow, large, full, reflexed......................... 60

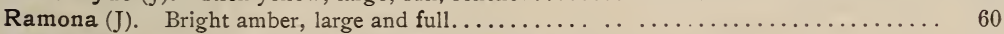

Rohallion (J). Dark chrome yellow, twisted petals...................... 125

Rose Laing (J). Violet crimson, outside silvery, early....................... 125

Robert Flowerday (J). Petals crimson inside, old gold outside, incurved.......... 100

Sabine Mea (C). Golden yellow, large, globular........................ 150

Shasta (J). Large spherical blooms of tubular white florets.................... 60

Snow Ball (J). Round white like a snowball .......................... 60

Souv. de Menier. Dark crimson maroon, reflexed....................... 100

Sunnyside (J). Flesh color changing to white........................ 100

Twilight (J). Lemon, changing to white, strong........................ 80

Tyro (J). Light Fawn, high flower, broad petals....................... 100

Ulysses. Single Magenta, outside lilac, incurving....................... 100

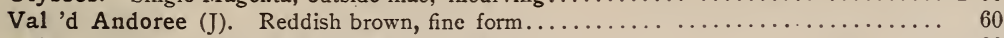

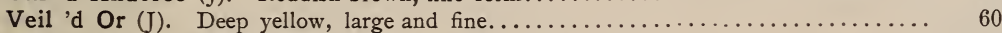

Vice-Pres. Audiguier (J). Soft rose, white points, large................. 200

Ville Marseilles (J). Dark pink, reflexed........................... 150

Violet Rose (J). Rosy violet, large incurved........................... 60 


\section{CHRYSANTHEMUMS-Continued.}

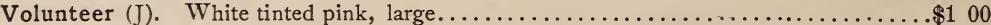

W. W. Lunt $(J)$. Lemon-yellow, large double...................... 100

W. H. Lincoln (J). Golden yellow, large, fine....................... 60

William Holmes $(\mathrm{J})$. Bright velvety crimson, medium size............... 150

William Lane $(\mathrm{J})$. Brick red, large flower.......................... 150

1200

Yonitza (C). White, incurved, fine form, large........................ 80

600

\section{For other Varieties see our General Catalogue.}

\section{Palms.}

Of Palms we have a fine large stock, clean and well grown. Have not been forced, but grown cold, consequently they are hardier, bear rough handling, and are better adapted for decorative purposes than those grown in strong heat. The kinds enumerated below are the best varieties in cultivation.

ARECA Baueri. A fine strong, dark foliaged palm ; strong, robust habit.

Good plants, 15 inch high............ \$0 75

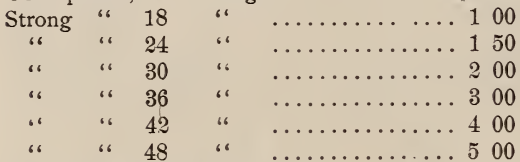

Extra large specimens, $5 \mathrm{ft} \ldots \ldots \ldots . \$ 6$ to 900

A. lutescens. Very graceful foliage and indispensable for decoration.

Nice plants, single stems, 12 inch.......

Good “ " " " 18 “

“ " “

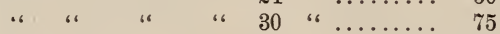

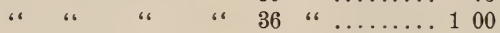

“ " $3 \quad 3$ in a pot, 12 “ $12 \ldots \ldots .60$

“ " " " “ 15 to 18 “ “

“ " " " 18 to 20 " “.......... 100

Fine bush plants, $31 / 2$ to $4 \mathrm{ft} \ldots \ldots \ldots . \$ 3$ to 400

4 to $4 \frac{1}{2} \mathrm{ft} . \ldots \ldots \ldots 4$ to 500

A. sapida. A strong growing palm, stands rough usage, fine.

Good plants, $21 / 2 \mathrm{ft}$. high............ 250

Strong and fine plants.............. 350

CHAMAEROPS elegans. A fine decorative palm hardy and tough.

Fine large spreading specimens, $5 \mathrm{ft} \ldots \ldots .800$ " " " " $6 \mathrm{ft} \ldots \ldots .1000$

Extra fine " " " 6 to $7 \mathrm{ft} . \ldots . .1200$

cocos Weddelliana. An elegant fine foliaged palm for mantel and table decorations.

Nice plants, 8 to 10 inches............ 35

“ " 12 " $12 \ldots \ldots \ldots \ldots \ldots \ldots, 50$

Extra fine specimens, $2 \frac{1}{2}$ to $3 \mathrm{ft} . \ldots \ldots \ldots . .300$

KENTIAS. A very popular and useful variety of palms, their long graceful foliage and erect habit make them almost indispensable for decorations.

K. Belmoreana.

Five to six leaves, 10 inch............. 75 15 " ................. 100
K. Pelmoreana-continued Each. Strong plants 24 inch............\$2 00 36 “ $\ldots \ldots \ldots \ldots \ldots, 400$

Extra large specimens, $5 \frac{1}{2}$ to $6 \mathrm{ft} \ldots \$ 15$ to 2500

K. Forsteriana.

Good young plants, $\quad 15$ inch........ 75

Fine plants, 4 to 5 leaves, 18 " $\ldots \ldots \ldots, 100$

Strong “" 24 “ $\quad \ldots \ldots \ldots .150$

" " 30 " $3 \ldots \ldots \ldots 200$

Fine well furnished plants 36 “ $\ldots \ldots \ldots 300$

Fine specimens, 5 to $6 \mathrm{ft} \ldots \ldots \ldots \ldots$ to 1000

Elegant “ 6 to $8 \mathrm{ft} \ldots \ldots \ldots \ldots \$ 12$ to 2000

LATANIA Borbonica. One of the most useful palms grown, with large fan-shaped foliage.

Good young plants, 4 to 5 leaves, 18 inches high........................ 50

Strong plants, 5 to 6 leaves, 20 inch...... 75

“ “ " " 24 “...... 100

Extra strong plants 7 to 8 leaves, 30 inch. $\$ 2$ to 300 Specimens, 8 to 10 leaves, 36 inch.... 4 to 500 9 to 11 leaves, 42 " $\ldots . .5$ to 600

PHOENIX. Date palms. Very useful and ornamental, strong upright growing, very effective.

P. Canariensis. Fine plants, $2 \frac{1}{2}$ to $3 \mathrm{ft}$. $\$ 3$ to 400

P. reclinata. Fine specimens, 3 to $3 \frac{1}{2} \mathrm{fc}$. 4 to 500

P. rupicola. One of the finest decorative palms in the world. Stock very scarce.

Plants to grow on ................ 85

Fine plants, 15 to 18 inch.......... $\$ 2$ to 300

Half specimens, $2 \frac{1}{2}$ to $3 \mathrm{ft} . \ldots \ldots \ldots \ldots 5$ to 800

P. Senegalensis. New glaucous species. Very graceful.

Fine specimens, 3 to $31 / 2 \mathrm{ft} . \ldots \ldots \ldots \ldots$ to 500

RHAPIS flabelliformis. A Chinese and Japanese palm of strong upright habit, with reed-like stems and divided leaves. A very effective variety for decorations, and standing more abuse and rough usage without getting unsightly than any palm in cultivation.

Nice plants, 2 to $2 \frac{1}{2} \mathrm{ft} . \ldots \ldots \ldots \ldots \ldots 200$

Good " two shoots, $21 / 2 \mathrm{ft} \ldots \ldots \ldots \ldots 300$

Fine " three to four shoots, $3 \mathrm{ft} . \ldots \ldots 500$

Extra large specimens. Very fine...\$\$ to 1000 


\section{Ferns and Selaginellas.}

This class of plants is extensively used for ornamenting dinner tables as well as for other decorative purposes. We have thousands of all the leading sorts at low prices, while our collection comprises all the best and valuable varieties.

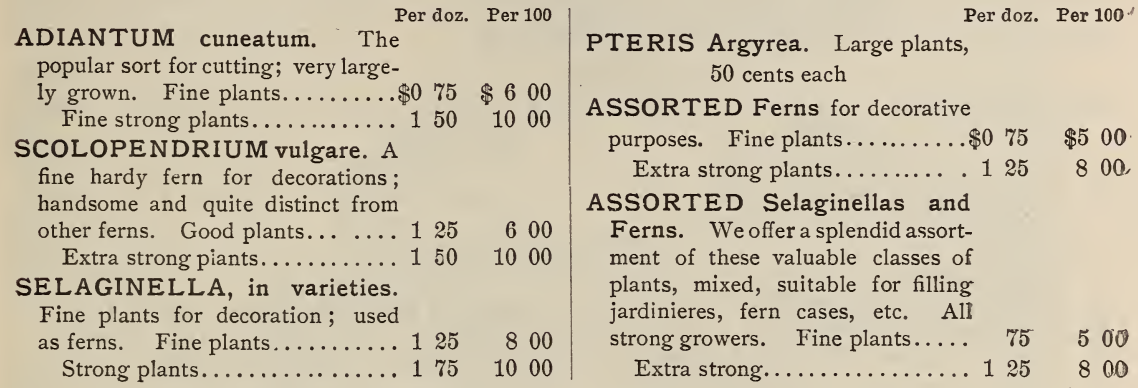

\section{TO FLORISTS.}

Our extensive stock includes all the rarer and less known desirable plants in all classes, as well as the commoner sorts which are known to every florist. We will take pleasure in giving estimates for filling greenhouses or will send descriptions of special plants of Palms, Tree Ferns, Cycads, Orchids, etc., which may be wanted.

We are also prepared to make selections of hardy herbaceous plants and bulbs for planting out around surburban residences, in beds and borders, or public parks and gardens, however large. Correspondence in regard to matters of this kind is invited.

\section{GUT FLOIKLERS.}

We are at all times prepared to send boxes of cut Orchid flowers, etc., on receipt of a letter or telegram. Some species or other are in blossom at any season of the year, and for special occasions the stock of the local florist is either inadequate or unsuitable. In the autumn and winter when our large collections of Chrysanthemums and Genistas are in blossom, we can supply cut flowers or specimen plants in flower of almost any description and in almost any quantity. We are glad to give information regarding what plants are in flower at any time. 


\section{Flowering and Foliage Plants.}

Our stock of these classes of plants is very large and embraces all the realy new and rare, as well as the best of the older varieties and we think the most complete in this country.

The following are the best and most profitable sorts for Florists' use, and we can supply these varieties in quantities, but those desiring a more extended list, can by applying for our Retail Catalogue, secure almost_anything desired, at a discount off retail prices.

ANTHURIUMS. Fine brilliant flowering plants. The flowers lasting a long time when cut.

A. Andreanum. Brilliant, dazzling scarlet, large flower.

Good strong plants........... 75c to $\$ 300$

A. Scherzerianum. Dwarf growing; very bright scarlet, smaller flowers....75c to 100

ASPARAGUS Plumosus. An elegant evergreen climber. Now in great demand by Florists' for decorative purposes; far superior to anything grown for trimming mantels, chandeliers, etc., heretofore very scarce : single strings when cut bringing generally from $\$ 10$ to $\$ 15$ per dozen wholesale in the Eastern markets, while stock plants for propagating could scarcely be had at any price. We offer strong imported plants, which are well established and in fine condition for making good strong strings the coming season. Ready after March 1st.

3 inch pot plants, fine, strong.. ? Prices by the 4 " " " " " " " " 6 " 4 hundred or 5 " " " " $"$ "

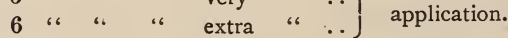

ASPARAGUS tenuissimus. Fine filmy foliage climber, useful for cutting, 5 in. pots

ALOCASIA Metallica. A very striking foliage plant, with bronze metallic leaves....

A. Sedenii. Peculiar arrow shaped cut foliage, light green with light silvery veins....... 125

A. Thibautiana. Heart shaped foliage, light green, veins and edges white.......... 150

ANTHURIUM Crystallinum. Dark velvety green foliage, light silvery veins.......

A. Grande. Bronzy green foliage, light

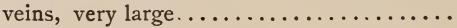

ARALIA Sieboldi. Palm like foliage, very tough, bears handling well, 4 inch pots,

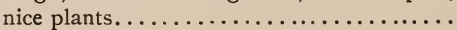

A. Sieboldi Variegata. Elegant green and silver foliage, very effective, fine strong plants...................... 10

A. Filicifolia. Fine cut foliage........

ASPIDISTRA. Half hardy foliage plants, very popular, long, bright leaves, succeed fine where other plants fail.
Each.

A. Lurida. Lurida. Green foliage, good plants.....\$0 50 Strong plants................... 75 Specimen plants...................250

A. Lurida Variegata. Elegant green and ivory white foliage $\ldots \ldots \ldots \ldots \ldots \ldots \ldots \ldots, 75$ Strong plants................. 100 Specimen plants...........\$3 00 to 1000

ARAUCARIA. A valuable tropical evergreen for decorative purposes, of very graceful and symmetrical growth.

A. Cookii. Fine plants to grow on....... 100

A. Excelsa. Fine plants in 5 inch pots.... 175 " Extra fine plants......... 350 “ Large specimens.....\$5 00 to 1000

BROMELIADS. We have a fine selection of these interesting plants in baskets. They are very striking and ornamental in decorations.................. to 300

Each Per Doz.

BEGONIAS, Best Rex Varieties. $\$ 0 \quad 25 \$ 250$

B. Argentea hirsuta. Center and edges green and chocolate, specked silver surrounding broad silver band.

B. Adolph Pollock. Silvery, very faintly edged and centered light green.

B. Comte de Limmingki. Silvery, finely edged and centered with greenish purple.

B. Duc de Brabant. Center and outer edge dark purplish green, inner bands of silver and light green.

B. Louis Closson. Dark chocolate edges and center, encircling dark pink silvery band, occasional pink spots in center and edge.

B. Lucie Closson. Purplish edges, center light green, mottled.

B. Mrs. A. Barron. Chocolate edges and center, surrounding crimson band, denticulated foliage.

B. Mad. Siebold. Silvery, shaded in center with green, edges mottled green with delicate pinkish shadings.

B. Rio de Ferd. Major. Silver, edged and centered partly with greenish velvet.

B. Voi Lactee. Silvery, center light green mottled dark green. 


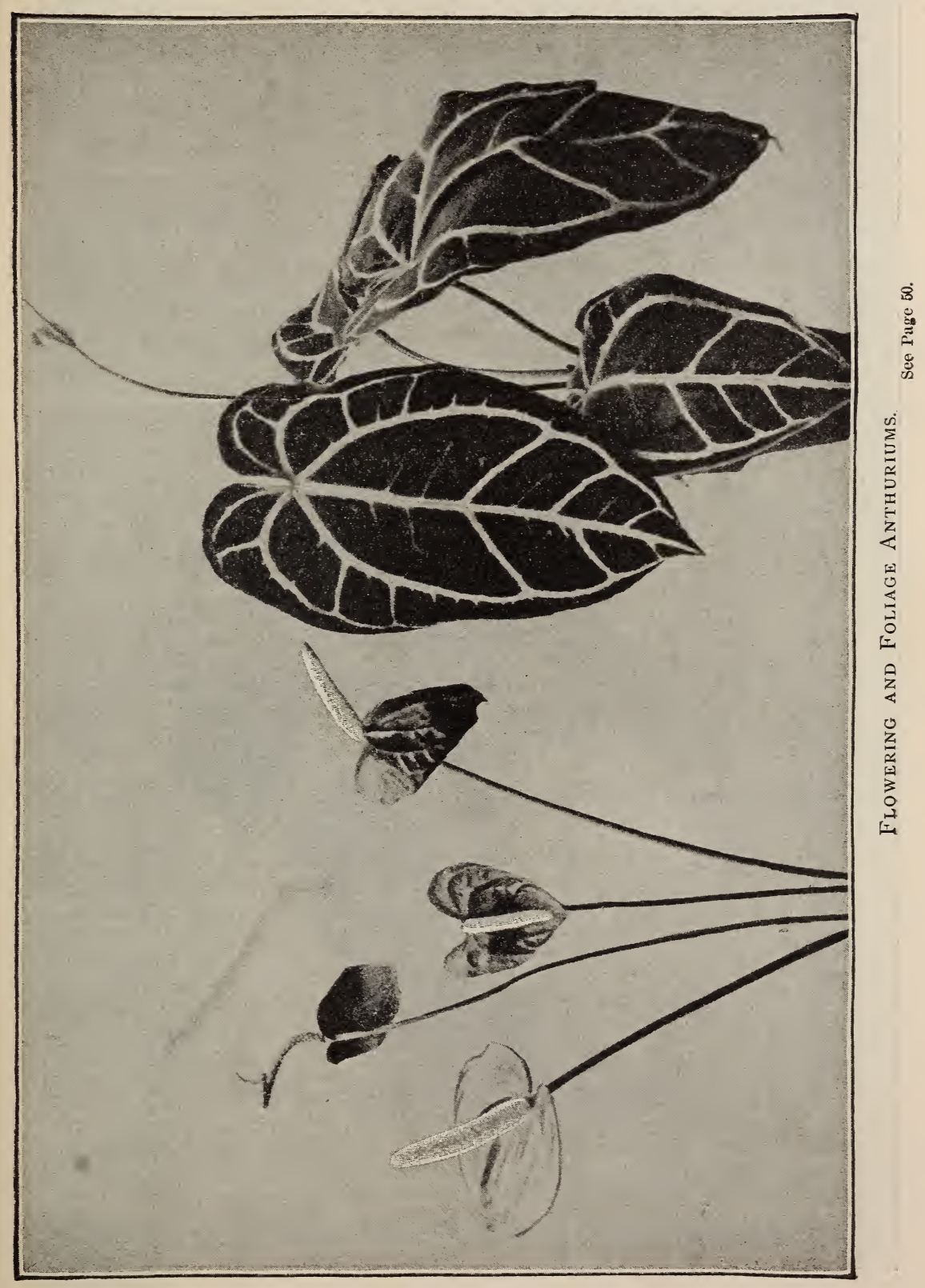




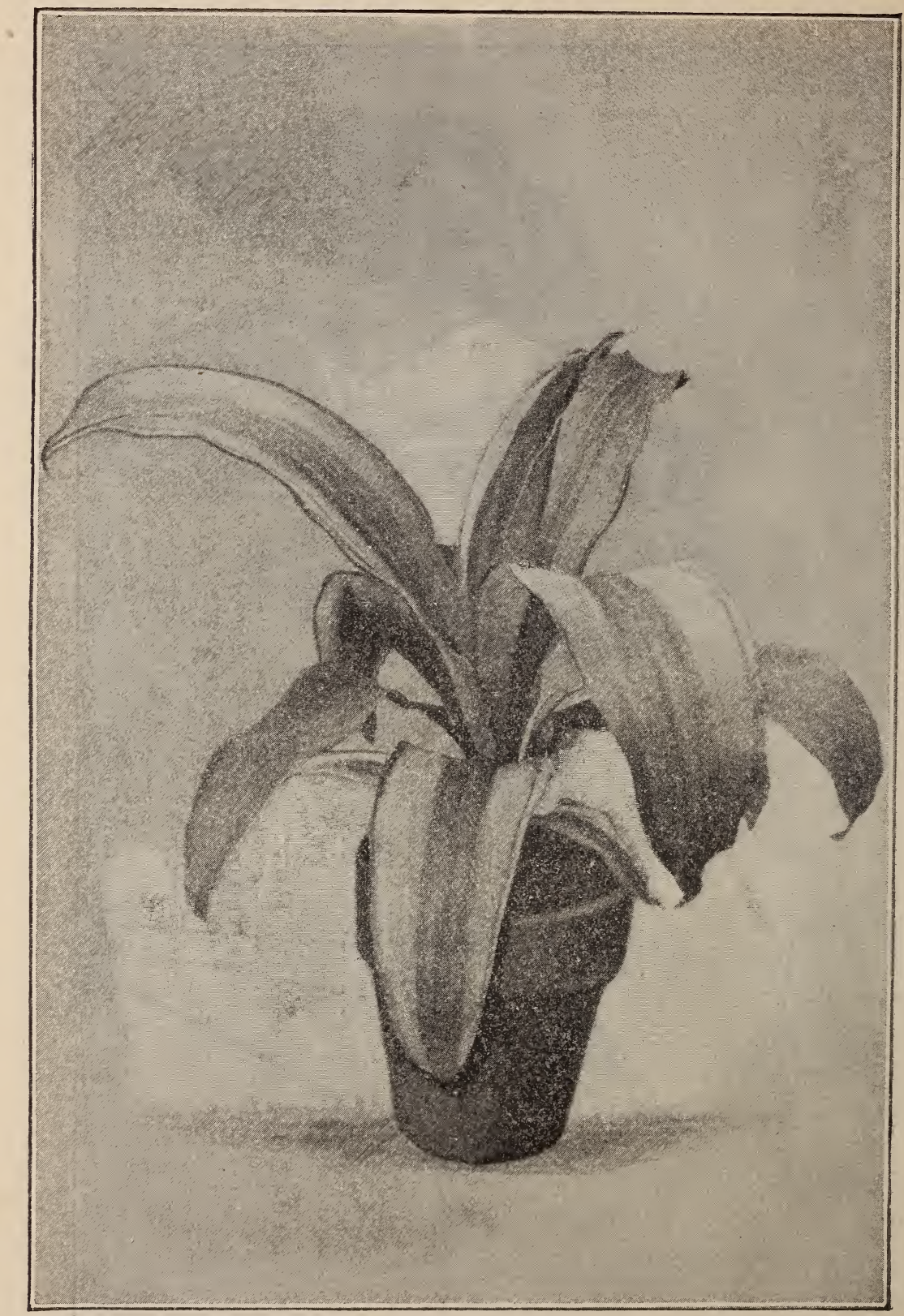

DRACÆNA LINDENI.

See Page 53. 
Flowering and Foliage Plants-Continued.

\section{BEGONIAS, Tuberous rooted.}

Our collection of these favorites is as fine as any in cultivation and far superior to many of the celebrated European growers. Per doz. Per 100 Fine large bulbs only, mixed, all colors. $\$ 125 \$ 900$

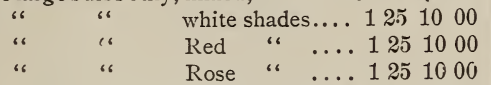
Rose " ....1 1251000 " Yellow \& bronze 1501200

CALADIUM. Elegant summer decorative plants. Our collection embraces many choice varieties................ $35 \mathrm{c}$ to. $\$ 050$

CISSUS discolor. An elegant greenhouse climber; foliage very rich in effect........

CROTONS. One of the most beautiful variegated foliage plants, of very easy culture. We offer many fine varieties...35c to

CHRYSANTHEMUM frustescens. The well-known Paris Daisy or Marguerite White.

CRYPTOMERIA elegans. A beautiful evergreen conifer.

Fine plants, 10 to 12 inch high......... 100

CYCAS revoluta. A very useful plant. Foliage always in demand for funeral desizns.

Small plants for growing on.............

Fine plants....................... 350

Large specimens............... $\$ 5$ to 1500

CYPERUS alternifolius. A pretty grass

like plant for sub-tropical groups.... .25̃c to

C. alternifolius variegatus. Pretty green and white foliage................ 100

DRACÆNA. This genus includes many grand plants for decoration, their broad leaves and stately habit make them useful and attractive.

D. Alsace-Lorraine. Fine dark leaves with rich crimson coloring............... 100

D. Cantrelli. Purplish ground, brilliant crimson tints...................... 125

D. Draco. True Dragon Tree. Erect green leaves..........................2 200

D. Lindeni. Drooping habit, leaves deep green, striped creamy white and yellow.

Plants, 12 inches high ............... 100

Extra strong plants................. 250 Specimens................... $\$ 4$ to 800

D. Massangeana. Similar to Lindeni in habit, foliage green striped pale yellow.... 125

D. Neo Caledonica. Massive erect foliage, dark green, purplish tinted. Stands rough usage equal to the hardiest palms ; fine for decorations ................... \$1 to 300

D. Mad. Heine. Upright habit. Green leaves edged ivory white............. 100

D. Terminalis. . Chocolate crimson, tinted carmine........................25c to 100
DIEFFENBACHIA magnifica. One of the best of the whole class. Dark green and white........................ $\$ 050$

D. in varieties................. 35 to 50

E RA N T HE M U M tuberculatum. A pretty, profuse blooming plant, with white flowers nearly $1 \frac{1}{2}$ inches across..........

EUCHARIS Amazonica. A fine fragrant winter blooming plant for florists, per doz...................... \$2 50

FICUS elastica. The well known Rubber Tree.

Good plants......................

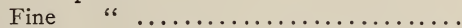

F. elastica variegata. An elegant variegated variety of the above. Small plants.. 250 Large good plants................. 500

GREVILLEA robusta. (Silk Oak.) A very pretty decorative plant with graceful foliage...................... 35 to ISOLEPIS gracilis. A pretty grassy plant useful for edgings, baskets, etc......

IMANTOPHYLLUM miniatum. (clivia nobilis). A fine flowering plant, producing orange red flowers freely throughout winter.

Fine plants $. \ldots \ldots \ldots \ldots \ldots \ldots \ldots \ldots, 100$

Strong " .......................... 200

LINUM trigynum. An invaluable house plant, producing a profusion of well opened bell-shaped yellow flowers..............

IMARANTA Zebrina. One of the oldest and most useful of this class of plants.....25 to

M. in varieties. Fine plants..........35 to PHRYNIUM variegatum. An elegant plant for decorative purposes, green and ivory white foliage..................

PANDANUS Utilis. Screw Pine. A very graceful plant, succeeds well in dwellings.

Four inch pot plants.................

P. Veitchii. Similar to the above in growth, with elegant green and white foliage.

Five inch pot plants............... 50

Very large fine specimens........... $\$ 5$ to 800

PANAX Victorie. A distinct and graceful plant, with lovely and elegant variegated green and white foliage............85 to

PHYLLOTAENIUM Lindeni. Of robust habit, light glossy green leaves, finely marked white................. $75 \mathrm{c}$ to 100

SARACENIAS. Curious herbaceous perennials, half hardy, remarkable for their pitcher shaped leaves.

S. Flava. Pale yellow pitchers and yellow flowers... ..................50c to 100

S. Drumondi. Beautiful colored tall upright pitchers, variegated red and white....\$1 to 300

ZAMIA integrifolia. A fine plant for decorative-purposes, similar to Cycas revoluta.

Nice plants . . . . . . . . . . . . . . . . 75

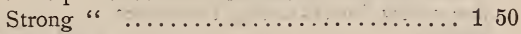

Extra strong plants................. 350 


\section{Orehids.}

Exactly according to our prediction, Orchids have taken a real hold upon the public favor, and there is no florist who can afford to be without them. Not only are they sold to the customer, but they act as an advertisement, and a two dollar Orchid plant in flower in a store or greenhouse will attract more attention than a two-hundred dollar painted sign. Besides being profitable, they will grow into valuable specimens, and thus in growing them alone they will give better returns for the money invested than any other class of plants. The six best Orchids we can recommend to anyone are Cattleya Trianæ, Cypripedium insigne, Coelogyne cristata, Cattleya Percivaliana, Cypripedium Harrisianum and Lycaste Skinnerii. The above varieties are indispensable, and below we give those that are generally grown for cut flowers, and which are found to be the most profitable.

CALANTHE vestita. A very useful terrestrial orchid, producing its pure white flowers in December, January and February..........\$0 $75 \$ \$ 70$

C. Dowiana. Undoubtely the finest Cattleya grown, flowering during the late summer and early autumn. The flowers are of a nankeen yellow color, lip rich purple, beautifully streaked with lines of gold........2 $250 \quad 2500$

C. intermedia. Free-flowering species, producing a fine spike of soft rose flowers, lip purple, during May, June and July............... 150

C. Mendelli. This fine species produces its pale pink flowers from May to July ; the lip is of a rich magenta. $175 \quad 1750$

C. Mossiæ. This grand orchid blooms from May to July. The large flowers vary from blush and lilac to deep rose ..................... 150

C. Percivalliana. Fine species, flowering from November to February. The flowers are deep rosy purple, with rich colored lip of deep crimson

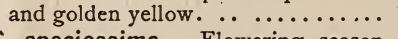

C. speciossima. Flowering season of this fine species is during the winter months. The flowers are very large of blush and rose colors......

CAT TLEYA Trianæ. This is the richest and most useful orchid in cultivation, flowering as it does from December to April, just when flowers are in great demand. The colors vary from pure white to blush, the lip having a bright crimson purple spot and yellow throat. Having been fortunate in importing a fine lot from our collector, Mr. John E. Lager, we offer them at the following low prices, all being finely established............\$95 per $100 \ldots 1251250$

Larger plants....\$195 "

COELOGYNE cristata. One of the finest winter-flowering orchids. Sprays of five to seven large white flowers are produced from January to March................... $100 \quad 1000$

Strong plants................. 2 $50 \quad 2500$

DENDROBIUM chrysotoxum. A grand species, producing spikes of fine yellow flowers during the winter months.................. 150
Each. Per doz

D. formosum giganteum. A fine, large white, winter-flowering Dendrobium; the blooms are handsome and showy................ \$1 $50 \$ 1500$

D. Nobile. One of the first and also one of the best orchids in cultivation. The flowers, which are white, tinged with pink, and with a lip blotched with crimson, are freely produced on straight stems in February and March. This is a standard variety. $100 \quad 1000$

DENDROBIUM thyrsiflorum.The white and yellow flowers of this fine species are produced in large, dense clusters in March and April.. 1500 D. Wardianum. The pseudo-bulbs
of this species are covered with white flowers, tipped with bright magenta and with a bright orange throat, during the winter months......... $150 \quad 1500$

Strong plants............... $250 \quad 2500$

LELIA albida. This is a very useful species, producing a long spike, bearing five to twelve white flowers, tinged with pink, from November to January............

L. Anceps. This well known species produces three to five large rose-colored flowers on a long stem, from November to January........... Strong plants................ $150 \quad 1500$

L. Arnoldiana. A very useful orchid of recent introduction; the flowers, which are produced during October, November and December, are of deep rose, five to fifteen being borne on a spike....................

purpurata. A grand species pro-
ducing from four to six large white flowers with purple lip, from May to July.......................

YCASTE aromatica As many
as eighteen yellow flowers are produced from one bulb during late winter and early spring...........

L. Skinneri. One of the most profuse flowering orchids in cultivation; as many as twelve large flowers sometimes being produced by one bulb; the flowers vary in shade from white to rose color, with lip blotched with rose or crimson; blooms same time as L. aromatica........ $100 \quad 1000$

Strong plants............. 2 $50 \quad 2500$ 


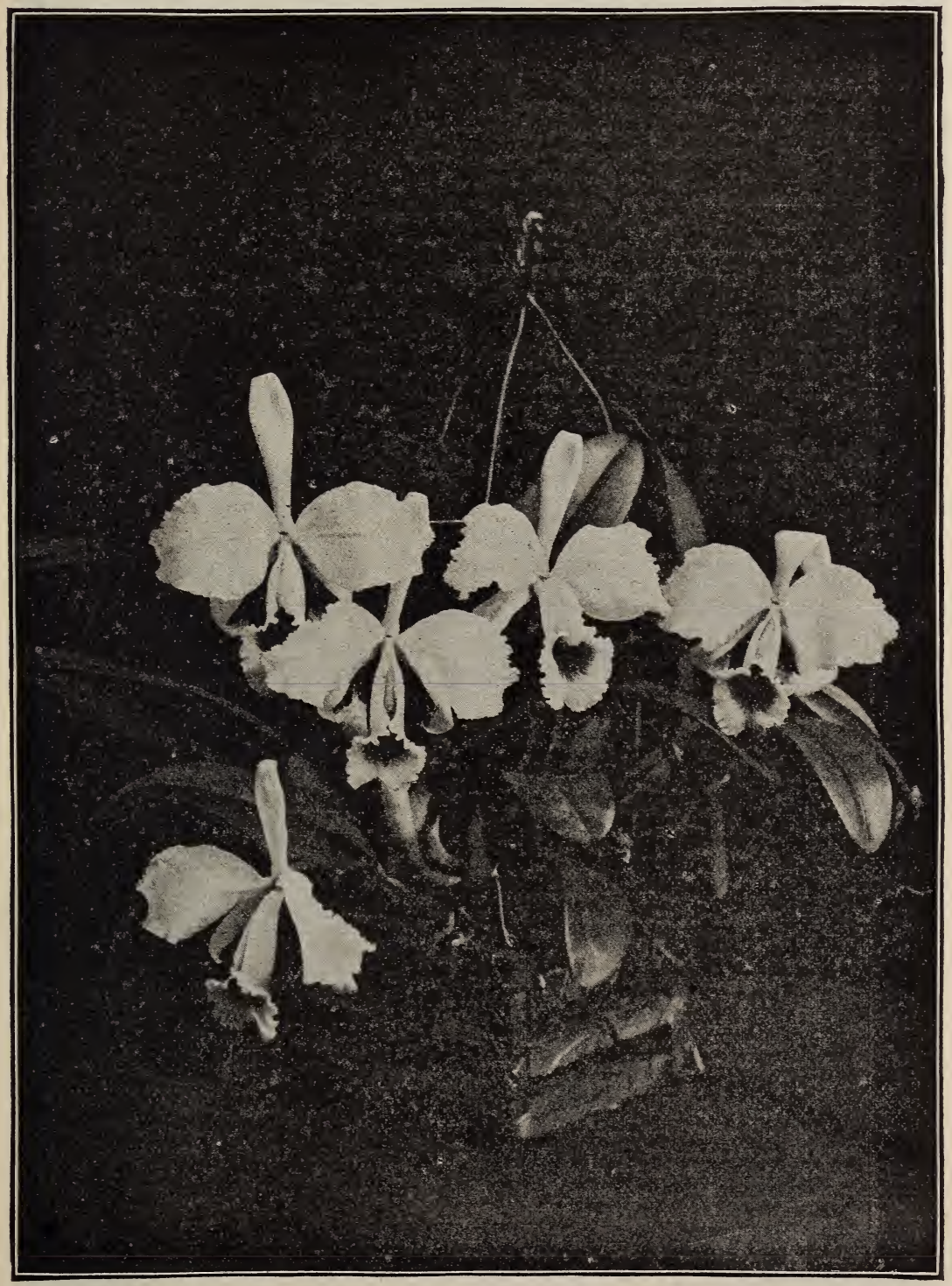

Cattleya Triane.

See Page 54. 


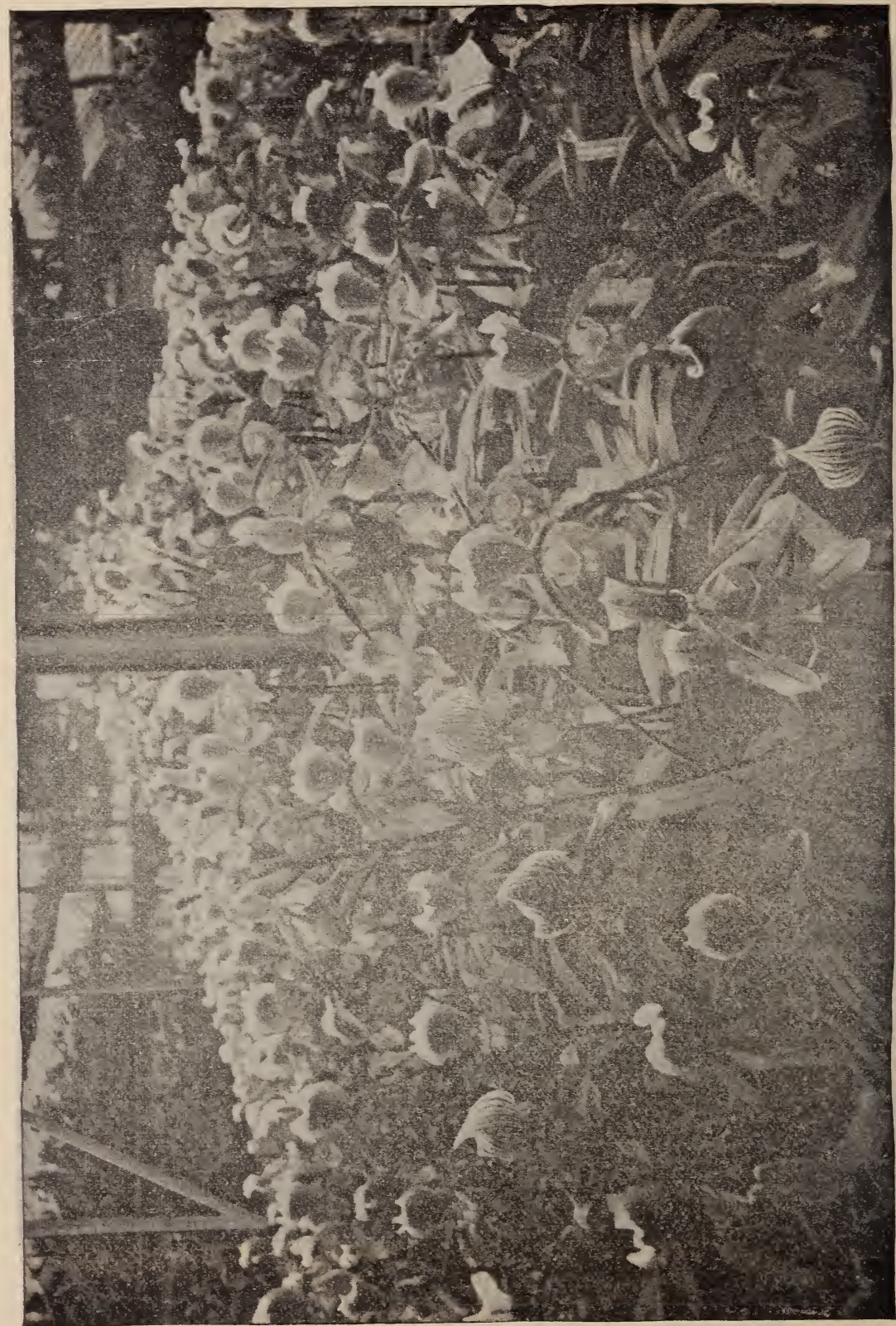

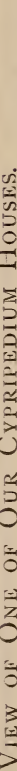




\section{ORCHIDS-Continued.}

ODONTOGLOSSUM citrosmum.

This species sends out a long, drooping spike of beautiful white or rose colored, lemon-scented flowers in Apriland May................ \$1 $00 \$ 1000$

O. crispum. Acknowledged to be one of the finest orchids in cultivation; long arching spikes of white flowers are produced from January

to April................... $100 \quad 1000$

Strong plants................ $250 \quad 2500$

O. Grande. Known as the "Baby Orchid." Cinnamon-brown and yellow flowers are produced from September to December... ......

O. pulchellum. A species of easy culture, producing very sweet-scented white flowers in the spring.....

O. Rossi majus. A very useful species, flowering during January, Feb-

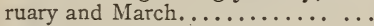

Strong plants................ $150 \quad \begin{array}{rrr}50 & 15 & 00\end{array}$

ONCIDIUM incurvum. Produces a large spike of pure white, marked with lilac and brown; flowers from May to July................. 150

O. ornithorynchum. A free-flowering species, producing its rose-purple flowers, with a perfume like the heliotrope, during the spring months.
$100 \quad 1000$

Each Per doz.$$
00
$$

75 1750
O. papilio. Known as the "Butterfly Orchid." The plant blooms nearly all the year round............ $\$ 175 \$ 1750$

O. tigrinum. A very showy sweetscented species, producing spikes of yellow and brown flowers in the autumn........................... species, producing large spikes of yellow and brown flowers in fall... $100 \quad 1000$

PHAJUS grandifolius. One of the oldest orchids. The flowers are white on the outside and reddishbrown inside. Produce its flowers on a long stem from February to April................... $150 \quad 1500$

PHAL ENOPSIS amabilis. One of the best orchids known for cut flowers. The large spikes of white are produced during the winter months..................2 $75 \quad 2750$

P. grandiflora. Another very useful species, producing its white flowers the same time as $P$. amabilis.....................2 $00 \quad 2000$

P. Schilleriana. The flowers of a fine rosy color, are produced at the same time as $P$. amabilis........4 $400 \quad 4000$

TRICOPILIA suavis. A very fine species, producing large white flowers, spotted with crimson, in the spring months................ $150 \quad 1500$

\section{Cypripediums.}

This very large class of orchids has attracted more attention than any other, and, therefore, we have put them under a separate heading. It is a well-known fact that Cypripediums are the easiest of all the Orchids to grow, and they last the longest in perfection. We had flowers last in perfect order for over four months on the plants, while when cut they last three to five weeks with a little care. The colors are rich and pleasing and combine well with roses and ferns, in fact with any other flowers. We have the largest, finest and most varied stock of these plants in the world, and recommend the following varieties to all Florists.

\section{CYPRIPEDIUM Ashburtoniæ.}

$A$ very free-growing hybrid, gener-

ally flowering twice a year...... . $\$ 1 \quad 75 \$ 1750$

C. Barbatum superbum. This popular species produces its flowers from April to Tuly................ $150 \quad 1500$

C. Boxallii. This very fine variety produces its flowers, which have a shiny appearance, as if varnished, from December to January....... $150 \quad 1500$

C. Dauthieri. This is another freegrowing and free-flowering hybrid; blooms in the autumn..........2 $250 \quad 2500$

C. Dominianum. This fine hybrid produces three to five flowers on a long stem in the autumn; the flowers are very useful for filling vases.. $1 \quad 75 \quad 1750$

C. Harrisianum. A grand hybrid, producing flowers twice, or even three times a year............. $150 \quad 1500$

C. Hirsutissimum. A very fine species, flowering during the Spring months....................... C. Insigne. The best known of all
orchids; flowers are produced from November to March, and last over twelve weeks on the plant, or three to four weeks when cut..........
C. Insigne album marginatum. A Each Per doz very fine variety of the common insigne, and flowering at the same time................... \$1 $75 \$ 1750$

C. Insigne maculatum. Stronger in growth, and with better flowers than the trpe $\ldots \ldots \ldots \ldots \ldots \ldots \ldots 150 \quad 1500$

C. Lawrenceanum. A grand species with beautiful variegated foliage; the flowers, which are on long stems are generally produced twice a year.

C. Longifolium. A free-growing species, which produces flowers on long stems, succeeding each other from May to July............. 1 \%5 1750

C. Roezlii. The habit and growth same as C. longiflorum; varies only in the color of the flower........ 1 i5 $17 \quad 50$

C. Sedeni. A fine hybrid, producing a long stem, on which large, pink flowers succeed each other from October to May................ $150 \quad 1500$

C. Venustum. A fine species, with beautiful variegated leaves; the flowers appear during the winter

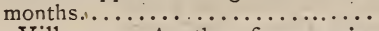

C. Villosum. Another fine species with shiny flowers, which are produced from January to April...... $150 \quad 1500$

\section{$75 \quad 750$}




\section{Hardy Perennials or Herbaceous Plants.}

We make a specialty of hardy herbaceous plants, and devote.acres of land to their culture. During the past year or two there has been a greatly increased demand for plants of this class, and there is much to be said in praise of them. With a good selection the garden is gay with their blossoms from the time snow leaves the ground in spring, until late autumn. We are continually adding fine novelties, both from our special collectors and from other sources.

Many species and varieties have proved excellent for forcing, and others that have not as yet been tried, will undoubtedly prove useful in the same direction. For florists and others purchasing plants to supply cut flowers, a large number of plants of few varieties are preferable to a few plants each of many varieties.

We send out none but healthy stock, true to name, and sure to give satisfaction. A personal visit to our nursery at any time during the summer is sure to be of interest.

ACHILLEA "The Pearl." The

flowers, which are borne upon erect foot-stalks, are of the finest white, and closely resemble a pompon chrysanthemum. Succeeds anywhere, and flowers almost the whole season. A grand acquisition for cutting......\$1 $00 \quad \$ 500$

A. multifolium rubrum. Very useful, producing heads of a deep lake rose color ................... 150

A. tomentosa. Flower heads flat, of an unusually good yellow, and remaining a long time in perfection; excellent for spring cutting....... 150

AGROSTEMMA coronaria. An erect branching plant, with handsome silvery foliage, covered in summer with bright pink flowers. A valuable border plant........... 150

A. coronaria alba. A variety of the above, with white flowers.......... 1501000

AJUGA alpina. A splendid bedding plant, the handsome foliage making a fine mat throughout the year; covered in spring with spikes of white flowers.............. 125

A. Genevensis. Taller growing than the last, bearing spikes of bright blue flowers in spring.......... 125

A. pyramidalis. An erect growing species, with spikes of blue and purple flowers in May and June ... 150

A. pyramidalis alba. A very pretty low-growing plant having white flowers produced in whorls during May and June. Height 6 inches....... 150

A. reptans. The creeping habit of this plant makes it valuable for carpeting; flowers in May......... 100

A. reptans alba. A variety of the above, with white flowers........, 150

ALYSSUM saxatile compactum. A good plant for early spring bedding, forming compact tufts bearing

ANEMONE Japonica. One of the most valuable fall flowering perennials, producing showy rose-colored flowers on long slender stems; useful for cutting or the open border... 100
A. Japonica alba. Flowers of the purest white, in late fall. A grand flower for cutting.............\$1 00

A. Japonica elegans. Similar to the type, but with broader leaves and pale rose-colored flowers, of ten 3 inches across................. 1501000

A. Pennsylvanica. Flowers pure white, on long, slender foot-stalks, produced in great profusion in early summer. A bright plant in borders, and excellent for cutting......... 100

A. sylvestris. Snowdrop windflower. Flowers pure satin white, fragrant, slightly drooping........ 100

AQUILEGIA Canadensis. Flowers scarlet and yellow, on slender branching stems. An early spring flower, of excellent habit......... 100

A. chrysantha. Flowers beautiful golden yellow. One of the finest of hardy perennials for the border.... 2 50

A. vulgaris alba. A stately plant, the nodding flowers borne on slender, erect stems. Showy in borders, and fine for early cutting........ 125

A. hybrids. All colors mixed...... 125

A R A B I S albida. An excellent plant for spring bedding, the dense tufts of white flowers appearing in April..................... 100

ARTEMISIA pontica. A good bedding plant, with silvery leaves and yellow flowers. Valuable for its aromatic fragrance. Excellent for florists to sell in pots............ 100

ASCLEPIAS tuberosa. One of the best border plants. Flowers a most brilliant orange, lasting long in perfection. Height 2 feet......... 125

ASTER Douglasii. Flowers purple, in August, on branching stems. 100

A. Novæ Angliæ. One of the best asters, of a tall, robust habit, bearing purple flowers in terminal clusters in September.............. 125

ASTER ptarmicoides. The finest white flowering species, blooming 


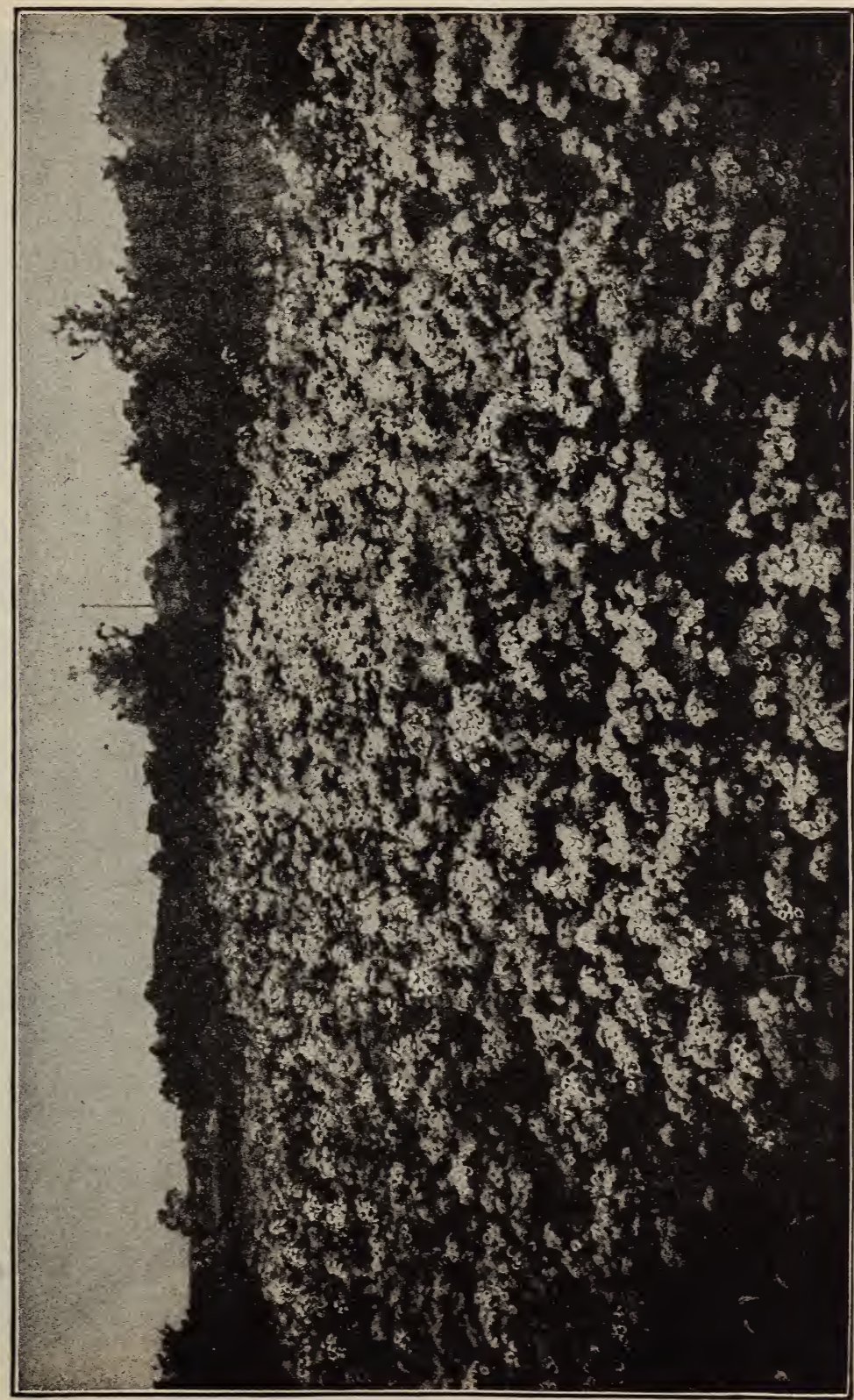

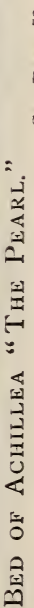




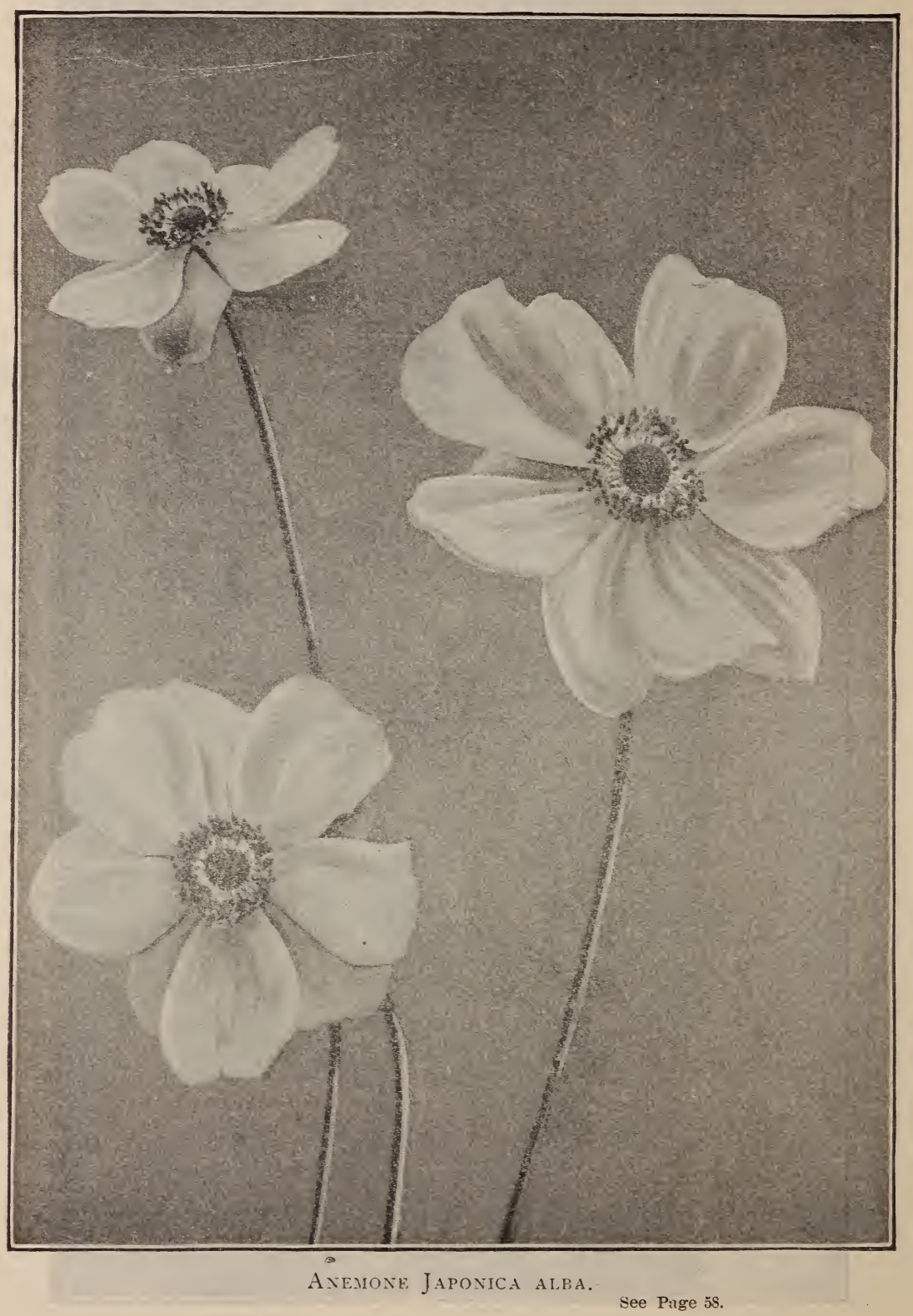


HARDY PERENNIALS-Continued.

A. Simplex. Flowers pale blue Per doz. Per 100 stems three to six feet high, sparingly branching..................\$1 $00 \$ 600$

A. in many Varieties. The brilliancy of these flowers and their late time of blooming make them especially valuable for cutting; they range through a great variety of shades and are in bloom through September and October..................

AUBRETIA græca. A lovely little spring blooming plant for rock works, etc., with light purple flowers forming a dense mass.......... 100

BAMBUSA Metake. A handsome tall growing bamboo grass, perfectly hardy; four to six feet high.....2 200

BAPTISIA australis. The finest tall blue flowering perennial. The foliage is handsome and the dark blue flowers hang in graceful racemes. 150

BERBERIS aquifolium. A low shrub, three to six feet high, with compound, spiny toothed leaves, and yellow flowers in crowded racemes, in spring .................... BOLTONIA asteroides. Flowers
light salmon colored, star shaped, disposed in a terminal panicle; blooms in August............. 125

BUXUS sempervirens. Common Box. A low growing evergreen shrub useful for borders to walks, etc. 150

CALLIRHOE involucrata. Flowers crimson, nearly two inches across, borne in a loose panicle in summer. 150

CALTHA palustris. The well known Marsh Marigold, which bears its bright yellow flowers in early spring. Succeeds best in a damp place.................... 100

CAMPANULA Carpathica. A low growing Campanula; large showy blue flowers borne all summer. 150

C. Carpathica alba. A very fine white variety............... 200

C. lactiflora. Flowers milky white, usually borne on three colored peduncles ; fine for the border, two to four feet................................

C. persicæfolia. A fine border
plant, two or three feet high, with large flowers of showy blue. Excellent for cut-flowers............. 150

C. persicæfolia alba. Similar to the preceding, but with white flowers... $150 \quad 1000$

C. persicæfolia alba plena. Very beautiful variety; double bell-shaped flowers of lasting quality when cut. 200

CARDAMINE pratensis plena. A very pretty purple flower, rising to over a foot in height......... 150

CASSIA Marylandica. A low growing shrub with pinnate leaves and yellow flowers in racemes...... $150 \quad 1000$

CLEMATIS. See page 69 of this catalogue.
COREOPSIS lanceolata. This is, without doubt, one of the finest hardy perennials. The flowers are produced in the greatest profusion from June until frost ; the branching wiry stems make it especially suitable for cutting............\$1 25

C. rosea. Flowers with rose red rays

and yellow disc................ growing branching species, with fine showy flowers, white, light and dark blue, in close racemes. A plant of very elegant habit, and fine for cutting....................... 175

D. mixed Varieties. Very useful and handsome border plants. The plants are of stately habit and make excellent centers for beds. The flower spikes hold a long time when cut........................ $150 \quad 900$

D., Double mixed Varieties...... $150 \quad 1000$

DIANTHUS barbatus. Very effective and showy border plant, and fine for cutting................. 125

D. deltoides. Flowers solitary, rose colored, borne in summer on stems six to nine inches high......... 125

D. Hispanicus. Large flowers of bright crimson, borne all the season. Excellent for cutting........... 125

D. plumarius plenus albus. White, double, the petals with jagged and toothed margins; sweet scented.... 125

D. plumarius plenus roseus. Similar to the preceding, but the flowers are purple or pink .......... 125

DICENTRA eximia. Drooping flowers of reddish purple borne in compound racemes in late spring and early summer.............. 125

D. spectabilis. The well known Bleeding Heart. An old and very ornamental border plant. . ..... 125

DIGITALIS gloxinoides. A well known ornamental plant; fine for massing or cutting............ $150 \quad 900$

D. gloxinoides alba. A white variety $175 \quad 1000$

D. purpurea alba. A fine white variety of the common foxglove. Very useful for planting in borders..... $150 \quad 900$

DORONICUM plantigineum excelsum. Very fine for winter forcing; height, three to four feet; flower heads yellow, four to five inches across. In the way of $D$. Caucasicum, but much finer............2 $200 \quad 1500$

ECHINACEA purpurea. A very showy plant, the bright rosy purple flowers succeeding each other for over two months. Useful for cutting $150 \quad 900$

\section{ERYNGIUM a methyst in um.} Flowers amethyst purple, in globose heads. Height, one to two feet.... $150 \quad 900$

ELYMUS glaucus. An ornamental grass, forming tall clumps covered with arching glaucous blue leaves.. 200 


\section{HARDY PERENNIALS-Continued.}

ERIGERON auranticus. A fine Per doz. Per 100

plant with bright orange colored asterlike flowers, two inches across. A good flower for cutting purposes... $\begin{array}{lllll} & 50 & \$ 8 & 00\end{array}$

E. glaucus. A fine border plant, growing two feet in height and producing numbers of lilac-rose rayed heads of flowers..............2 $00 \quad 1200$

ERYSIMUM pulchellum. A prostrate plant with numerous pale yellow flowers in early summer........ $175 \quad 1200$

E U L A L I A Japonica variegata. A handsome grass, growing five to eight feet in height, the green leaves marked with white bands ........2 $200 \quad 1500$

E. Japonica zebrina. A very handsome form with yellow bars running transversely across the leaves......2 $250 \quad 1800$

EUONYMUS radicans. A handsome shrubby foliage plant, well suited for trailing over rocks, stumps, etc. Very free growing and useful........................ $150 \quad 1000$

E. radicans variegata. A variety of the preceding with fine variegated foliage. When trimmed it makes a good stiff border............... $150 \quad 1000$

EUPHORBIA corollata. Flowers white at the end of the branches; plant with a milky juice.......... 1501000

FUNKIA cordata. Flowers white ; leaves broad, pale green......... 125

F. cordata variegata. A variety with the leaves marked with white.. $150 \quad 1000$

F. Lanceolata. Flowers white, the leaves longer and narrower than in the preceding variety.......... 100

F. lanceolata variegata. A variety with the leaves marked with white.. 125

F. undulata variegata. A very fine border plant with crinkly foliage, variegated with creamy white. Useful for edging................ $150 \quad 1000$

G A I L L A R I A grandiflora. Flowers yellow with annular ring of crimson. Are all summer bloomers, excellent for cutting............. 1501000

G L E C H O M A hederacea variegata. A good plant for hanging baskets. The leaves are prettily variegated; the habit of the plant is trailing................... 100

GERANIUM platypetalum. A very showy border plant, the deep violet flowers being borne in great profusion. Excellent for cutting in E L I A NTHUS Maximilianii. E L I A NTHUS Maximilianii.
A large growing and very free flowering specits remaining in bloom till late autumn; a valuable species for cutting purposes........... 200

H. multiflorus plenus. An autumn bloomer with large double flowers; a valuable species for cutting...... 150

H. multiflorus grandiplenus. An improved variety of the last, with very showy flowers not unlike a yellow chrysanthemum, 4 to 5 in. across. 200
H. orgyalis. Flower heads yellow, Per doz. Per 100 rather small, but very numerous in a large panicle; one of the best autumn flowering plants.........\$2 $50 \$ 1800$

H. rigidus semiplenus. Semi-double disc and rays, rich dark yellow; one of the best for cutting..........2 $00 \quad 1200$

HELIOPSIS lævis. A valuable plant, blooming steadily from nidsmmmer to frost ; flowers large and borne in great profusion ; invaluahle for cutting...................... cies is very valuable for forcing in winter; when covered with a frame the beautiful white flowers may be had in bloom at Christmas.......2 $200 \quad 1200$

H. maximus. Larger flowered than

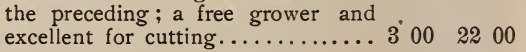

HEMEROCALLIS flava. A beautiful plant, admirable for forcing; the sweet scented lemon-yellow flowers succeed each other for some time............................ fulvers a tawny color, borne all summer................

HEPATICA angulosa. An exceedingly bright colored species, the large deep blue flowers appearing in early spring before the leaves...... . ing border plant, with handsome foliage and large pinkish white flowers in early summer................ known and noble plant we have an immense stock, grown from the finest double strain of seed, which we are pleased to offer, but do not guarantee that each plant will come true to color or double, though at the same time fully $9 . \bar{j}$ per cent will do this. Assorted colors........... best of the hardy candytufts. Flowers white, large, fragrant; good bloomer........................... An exceedingly pretty dwarf species, well adapied for edgings; flowers pale blue with white markings.................

I. cuprea. Flowers dull yellow, borne on stems often three feet high, in April and May...............2 200 1500

I. flavescens. Flowers lemon-yellow; a fine variety for borders, its flower stalks, two to three feet high, being produced in May..........

Germanica, varieties mixed. A
highly ornamental species; the stately habit, the brilliancy and variety of the shades and markings make it invaluable for ornamental or cutting purposes.................... $050 \leftleftarrows 250$

I. Kæmpferii Varieties. Mixed. The flowers of this remarkable species are broad and flat and present great variety of color. Height 4 feet.... $150 \quad 600$ 


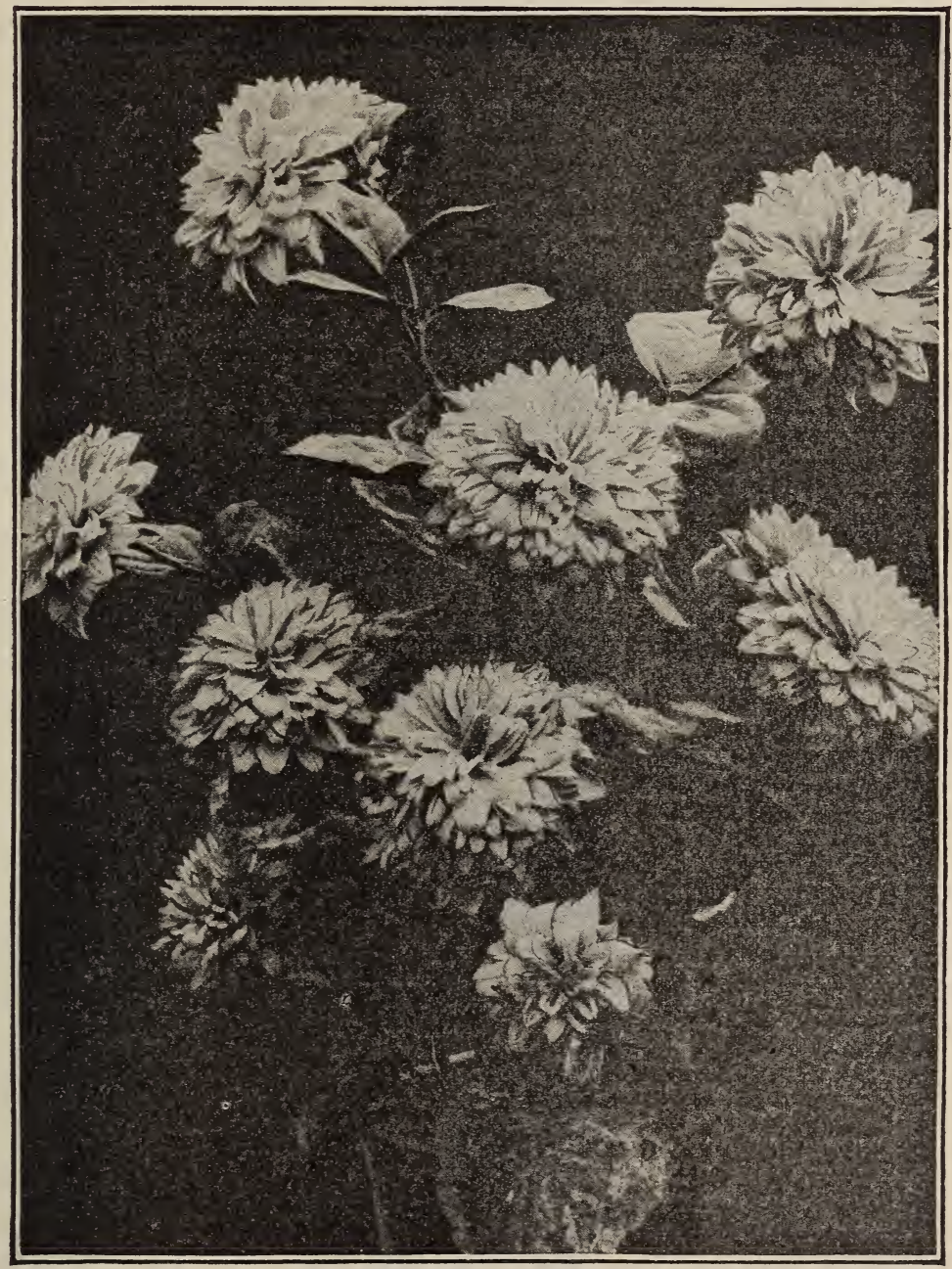

Helianthus Multiflorus Plenus. 


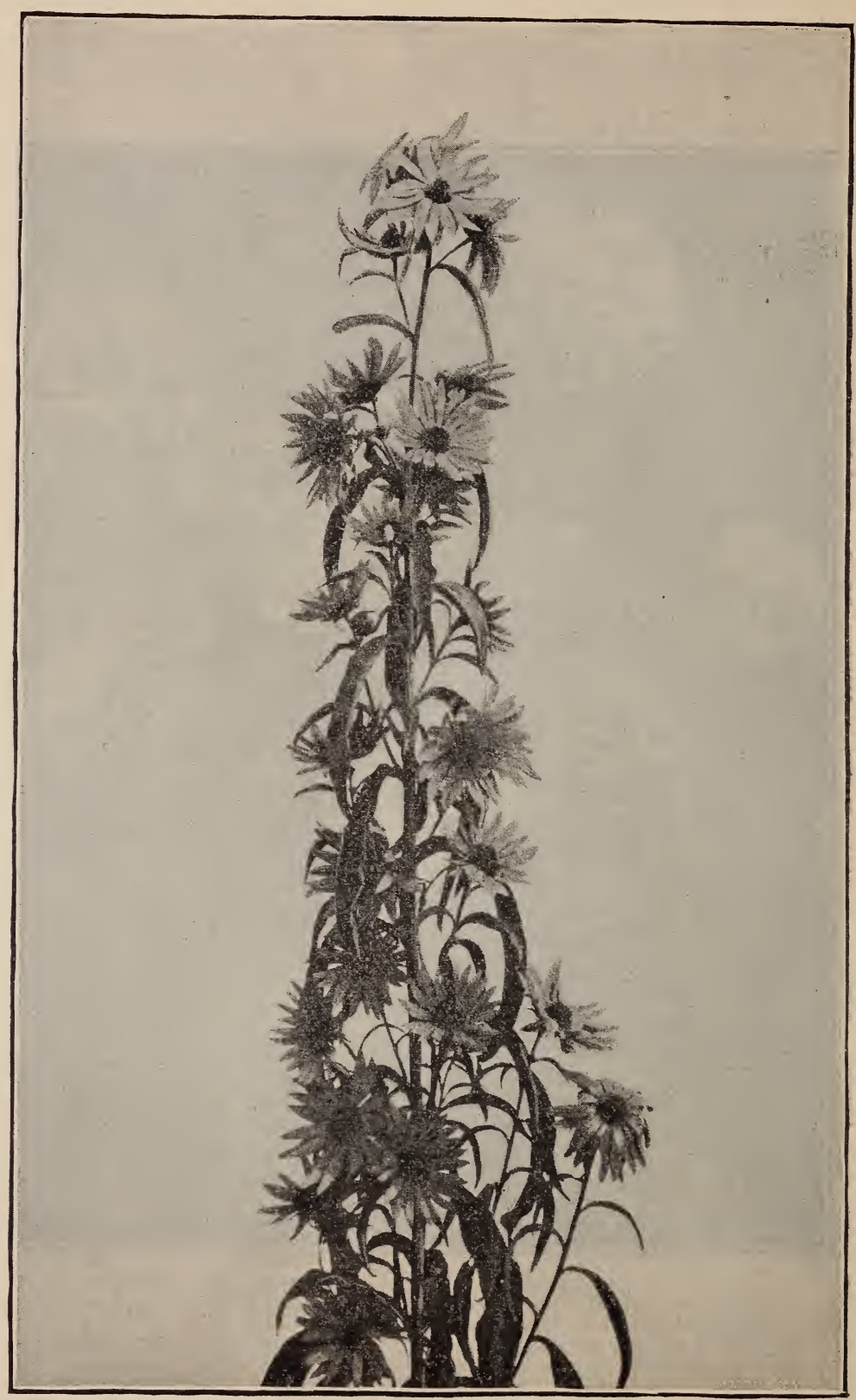

Helianthus Maximilianit. See Page 62 . 
HARDY PEREXNIALS-Continued.

Per doz. Per 100

Iris nudicaule. A short, stout growing variety with violet purple flowers. $\$ 1 \quad 50 \$ 1000$

I. pseudo-acorus. Flowers large, bright yellow, produced in early

Spring. Stem two to three feet high $150 \quad 1000$

I. pseudo-acorus variegata. A variety of the preceding with leaves marked with lighter shade ........200 1500

I. setosa. Flowers clustered, bright lilac; stems two to three feet high branched................... 1501000

I. Sibirica sanguinea. A fine species with narrow leaves and flowers of a very fine dark blue; one of the best of all the Irises............ $150 \quad 1000$

LEUCANTHEMUM lacustre. Large, daisy-like yellow flowers, produced in autumn................ plant with erect stems and dense spikes of purple flowers in late

L. scariosa. Flower heads one inch across, bright purple, in tall spikes..

LOTUS corniculatus. Flowers bright yellow, fading to orange, produced in late summer and autumn; a very handsome plant for growing on rock-work.................. with heads of bright pink flowers in spring and summer; good for low borders....................... ored tall perennial; the flower-heads are bright scarlet and are excellent for cutting................ $150 \quad 1000$

L. viscaria splendens. Flowers deep red, stems growing about one foot high.................. $150 \quad 800$

LYSIMACHIA Nummularia. An elegant creeping plant with a profusion of bright yellow flowers rising from a carpet of green; will grow where grass cannot..............

MONARDA didyma. A sweetscented herb with crimson flowerheads. Excellent for borders and for cutting. Is in flower for at least three months.................. MYOSOTIS palustris semper-
florens. An improved variety of the well-known forget-me-not. An excellent plant for low borders, as it flowers the whole season ........ 150

M. elegantissima. A fine spring flowering plant of compact habit. Excellent for forcing and spring bedding...................... Flowers

deep yellow, borne on spicate racemes from June to September......... $150 \quad \& 00$

OPUNTIA Rafinesquii. A hardy cactus with broad, evergreen leaves and yellow flowers. Stems and leaves covered with spines........ 150

O. vulgaris. Similiar to the last but not so spiny.............. 150
PACHYSANDRA terminalis. An Per.doz. Per 100 excellent bedding plant, increasing rapidly and making a bright evergreen carpet................. \$3,00 $\$ 1800$

$P$ IEONIA albiflora, in varieties. Mixed. Fine varieties of this showy perennial, which we grow in large quantities..........................

P. tenuifolia. One of the finest
species; flowers large, bright red, with fern-like foliage. Very early, and excellent for forcing........2 251750

Pæonias. Strong clumps for forcing ; assorted ; best six varieties, including whites..........Each 75 cts. $750 \quad 6000$ Extra strong clumps... Each $\$ 1 \ldots 10 \quad \ldots 0 \quad 8000$

PAPAVER alpinum. Similar to $P$. nudicaule, but more dwarf in habit. The flowers are white, yellow and rose tinted...............2 $00 \quad 1500$

P. bracteatum. A very showy flower; blood-red, with a black center...................20 $00 \quad 12=00$

P. involucratum maximum. A fine plant of medium height, bearing red flowers with dark spots at the base of the petals............. $200 \quad 1200$

P. nudicaule. A very pretty poppy, destined to become a universal favorite. The foliage is fern-like and the graceful stems bear yellow, white and saffron flowers. Very useful for cutting purposes............... 1 50 1000

P. orientale. A tall growing poppy with immense flowers of bright scarlet, with a dark purple spot at the base of each petal........... $200 \quad 1200$

$P$. orientale semi-plenum. A fine variety with an increased number of petals. A great acquisition....... 2 $50 \quad 1500$

P. pilosum. Flowers large, bright scarlet, with a white spot at the base of the petals................ $200 \quad 1200$

PARDANTHUS sinensis. A fine border plant with orange colored fowers in August and September... 100

PASSIFLORA incarnata. A fine hardy Passion Flower, bearing blue flowers in spring. A fine climber... 125

PENTSTEMON diffusus. A valuable species, with large blue flowers. The best of the hardy pentstemons.. $200 \quad 1200$

P. digitalis. A very ornamental plant, bearing tall spikes of white flowers in summer.............2 $00 \quad 1200$

PENTSTEMON pubescens. A smaller species, with spikes of lilac flowers.................. \$1 50 \$ 00

PHALARIS arundinaces variegata. A very ornamental grass with striped leaves. Useful for massing or cutting............... 10

PHLOX amœna. Unsurpassed for spring bedding, the foliage being entirely hidden under the profusion of bright pink bloom ............. 100

P. Carolina. A beautiful pink-purple flowered species, bearing large clusters of flowers nearly all summer. Valuable for cutting............. $150 \quad 80 \quad 8,00$ 


\section{HARDY PEREXYIALS-Continned.}

$P$. paniculata, in varieties. $U n-$ doubtedly one of the most showy of hardy perennials. For display of bloom or for cutting, nothing is better than a selection from this handsome section.............. \$1 50 \$ $\$ 0$

P. paniculata, "The Pearl." A pure white dwarf variety. The flowers do not shatter, and its compact heads are fine for cutting; a grand novelty, and bound to find faror with the florists.............. $150 \quad 1000$

P. subulata. A prostrate species, making an excellent spring bedder. Flowers a pale pink, from moss-like foliage.................... 100

PHYSOSTEGIA Virginica. A handsome border plant, with onesided racemes of purple flowers,

produced all summer.............. $100 \quad 600$
POLEMONIUM cœruleum. A very useful border plant. Flowers on tall spikes in early spring; in color a good blue, showing the yellow stamens........................... tufts of fine foliage, covered in early
ture spring with showy blue flowers.....

POTENTILLA, double hybrids. Several varieties with red and yellow flowers: fine for borders or rockwork .................. 150

P. fruticosa. A dwarf growing shrub which bears bright yellow flowers in early summer............. 150

PRIMULA acaulis, in varieties. The flowers, yellow, pink or white, are borne singly in early spring.... 1 i5 elatior, in varieties. Flowers vary from yellow to deep maroon. Very suitable for planting in rockwork or borders............... 1 i5

P. Japonica. Flowers of various colors, pink, white, lilac, dark red, etc. Scapes one to two feet high....... 250 150

P. Sieboldii, in varieties. A grand Japanese hardy primrose varying in color from white to deep rose and purple...................... varieties. This is an excellent plant for cutting purposes. The shades of color run from white to crimson, and the flowers are both double and single.

Single varieties ............ $200 \quad 1500$
Double varieties (to color, in-

cluding white).......... 250 1800

P. uliginosum. A grand plant for fall cutting, growing 4 feet high and covered with a profusion of large white flowers. (See cut page 7 ).........
UDBECKIA laciniata. A tall

RUDBECKIA laciniata. A tall growing species with large, showy
flowers. Excellent for tall borders and for cutting............... $150 \quad 1000$

R. maxima. A tall growing species, with bright yellow flowers........2 $200 \quad 1200$
Par doz. Per 100

R. speciosa. A fine plant for cutting or massing. Flowers with orange colored rays and a black disc. Height, 2 to 3 feet............. $150 \$ 900$

R. sub-tomentosa. A tall species, with handsome broad leaves and an abundance of showy yellow flowers

all through the summer...........
RUELLIA ciliosa. A pretty prostate plant, effective in low borders, producing abundant lilac flowers in late summer.................

SALVIA pratensis. Flowers bright blue, about an inch long; borne on spikes in summer................

The pretty white poppy-like flowers appear in earliest spring before the leaves. Useful for borders....... $100 \quad 600$

SCHIZOSTYLIS coccinea. A late fall flowering perennial, with blood-red flowers on an erect spike.

SEDUM Fabarium. With the habit of $S$. spectabile, but with dark colored leaves; afine plant.......2 $200 \quad 1200$

S. spectabile. A very fine plant for borders and edging; leaves broad and showy. Flowers produced in September in rosy-purple clusters.

V. spectabile album. White variety of the preceding............. $175 \quad 1000$

SILPHIUM perfoliatum. A large leafed, stout growing composite, with large clear lemon-yellow flow: ers. The plant is corered with blossoms from the middle of summer through the season............. $\$ 200 \$ 1200$

SOLIDAGO, in several varieties. Very showy for massing or cutting

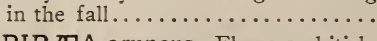
150800

SPIR IA aruncus. Flowers whitish, in many slender spikes, disposed in a compound panicle.............

S. filipendula flore pleno. Flowers,
white, borne in loose cymes in June and July................. $150 \quad 1000$

S. Japonica. A well-known and favorite plant for forcing in March. Strong clumps................

S. Japonica aurea variegata. A fine variety, with yellow markings on the leaves; very effective........ 2 $00 \quad 1200$

S. Japonica grandiflora. An excellent and undoubted improvement on the old form. Very lasting, and of a fleecy whiteness; invaluable for cutting.................. 1 75 1200

S. Japonica purpurea. A variety with purplish leaves; a very striking plant....................

S. palmata. A beautiful species, with sweet-scented crimson flowers. An exceedingly useful plant for cutting.................. $150 \quad 1000$

S. ulmaria. Flowers white, in compound cymes in July and August... $150 \quad \& 00$ 


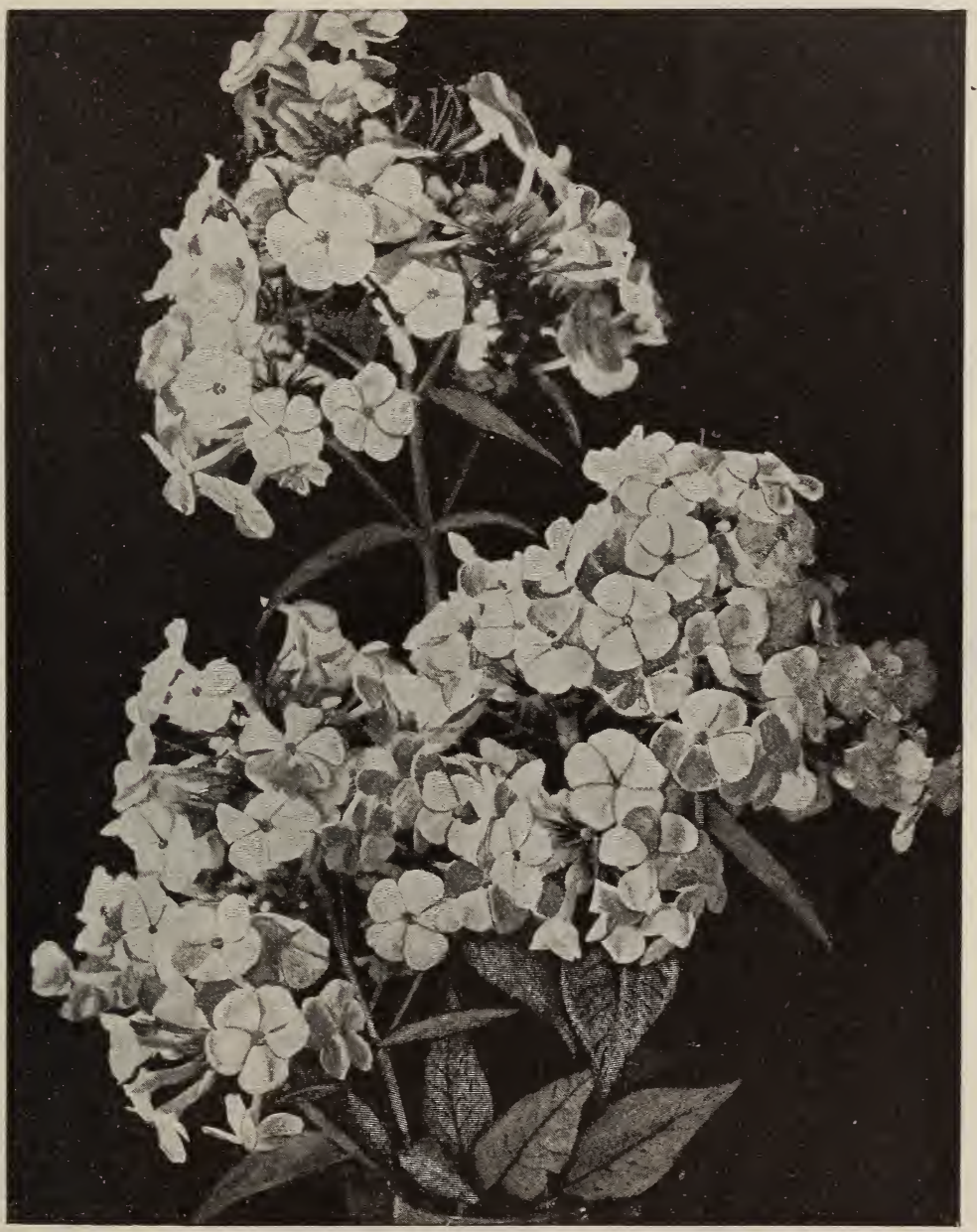

PHLOX "THE PEARL." 


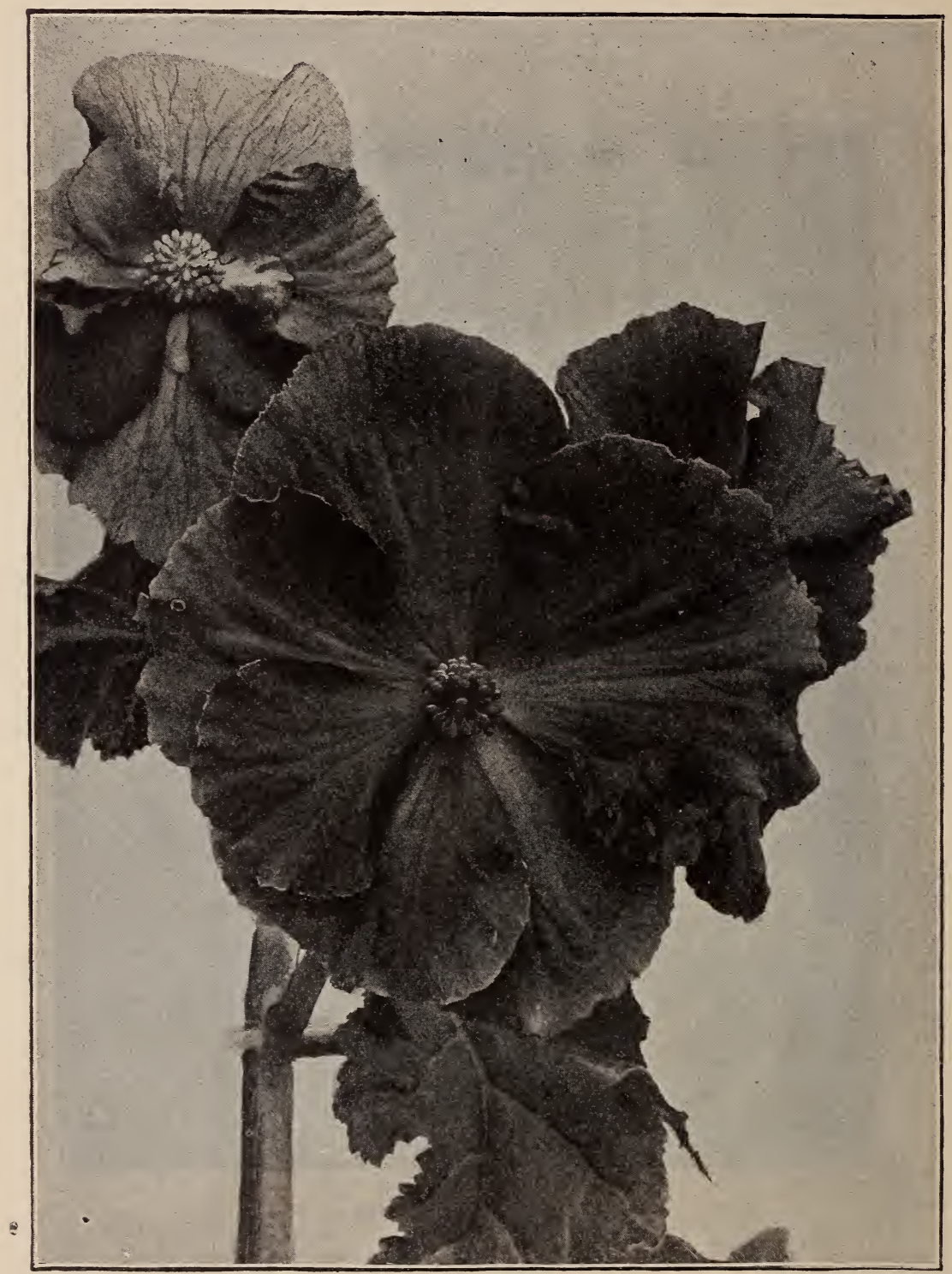

Our Superb Strain of Tuberous Rooted Begonias. seed, Page 5. Tubers, Page 53. 
HARDY PERENIALS-Continued.

STENACTIS speciosa. A very Per doz. Per 100 showy plant, producing numerous blue, aster-like flowers in summer. Height, $1 \frac{1}{2}$ feet.............. \$1 $50 \$ 1000$

STOKESIA cyanea. A fine, lateflowering plant, with large and showy flcwers of a beautiful blue color.... $1775 \quad 1200$

TRADESCANTIA pilosa. Similar to the next, but with somewhat broader leaves and darker flowers... $125 \quad 800$

T. Virginica. Flowers an inch in t diameter; brilliant purple, borne in a terminal and axillary cymes; blooming from March to May...... $200 \quad 1500$

T. Virginica alba. A fine variety, with pure white flowers......... $100 \quad 1000$

T R I L L I U grandiforum. A very handsome lily-like spring flower, the large, white blossoms rising from three dark green leaves. Fine for forcing and cutting..............

TUN ICA Saxifraga. Flowers pink, appearing in July. Excellent for planting on rock work........2 0
$75 \quad 600$

1500
VERONICA rupestris. Fine pros-

Per doz. Per 100 trate species, with blue flowers; unexcelled for edging or covering of rocks....................... \$1 $00 \quad \$ 600$

V. spicata. A showy plant, bearing handsome spikes of blue flowers in summer. ................ $100 \quad 600$

VINCA minor. An excellent trailing plant for rock-work, producing purple-blue flowers in early spring.. $100 \quad \begin{array}{llll}0 & 0 & 0\end{array}$

V. minor alba. A variety with white flowers.................... 125800

V. minor alba plena. With double white flowers................ 125800

V. minor purpurea plena. With double purple-blue flowers........ 125800

V. minor folia variegata. A variety with variegated leaves............ $1 \quad 800$

VIOLA cucullata. The variegated form of the common violet; flowers large, white and biue........... $100 \quad 600$

V. cucullata alba. Pure white variety; fine for edging.......... $125 \quad 800$

\section{Clematis.}

We have a fine stock of this most beautiful of climbing plants. The many colors which are now produced by the various hybrids, white, blue, purple, scarlet, in many shades, and the large size of the flowers, together with its hardiness, climbing habit and free-blooming, make them universal favorites.

Any of the following varieties, strong plants, our selection, 35 cents each; customer's selection, 40 cents each.

\section{Clematis Jackmanni Varieties.}

Anderson Henryii. White.

Countess of Lovelace. Bluish lilac, double,

Duke of Edinburgh. Rich purple, large.

Gem. Deep lavender, very fine.

Jackmanni. The, best known variety, with flowers of a rich purple, with green stamens.

$\mathrm{J}$ a ckmanni superba. Deeper and larger than
Gypsy Queen. Velvet purple.

Lord Neville. Rich dark blue.

Rubro Violacea. Maroon shaded purple.

Sir Garnet Wolesley. Bluish shaded bronze.

Star of India. Violet purple, with rosy band.

William Kennet. Deep purple, changing to lavender.

\section{Special Imported Stock for Florists}

We would especially call the attention of the trade throughout the country to the fact that we are one of the largest importing houses in this country_for the following for Fall delivery :

\section{Azaleas. Indica Varieties.}

These we import in all sizes, and of the very best selected New York forcing varieties only. No casestuff_handled under any circumstances.

First Size.

27 to 30 inches in circumference.

Per doz. Per 100

Second Size. 35 to 45

Third Size. 45 to 60

Fourth Size. 60 to 75

"6

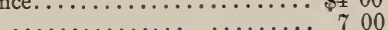

$\$ 3000$

5000

Extra Large Specimens.

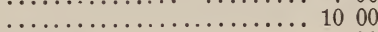

7500

9500

In selecting a suitable size we would suggest that florists take a good portion of the second size, that being, the size at a low price, and, will be sure to give satisfaction. 


\section{SPECIAL IMPORTED STOCK FOR FLORISTS-Continued.}

\section{Azalea. Mollis Varieties.}

We handle of these favorites the very best only, of the forcing and effective planting sorts.

Strong Bushes................................... \$6 $00 \quad \$ 4500$

\section{Rhododendrons.}

Our stock of these is now fine, and we continually add all the old and best of the tried new sorts, and only tested kinds for show and hardiness.

Strong Bushes................................. P10 00 . $\$ 7500$

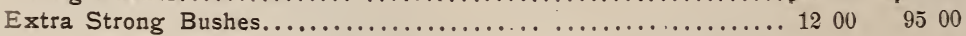

\section{Laurus Nobilis. Bay Tree.}

These beautiful and hardy decorative plants are now getting in great demand, and we annually import a selected lot of fine specimens, both pyramids and standards.

Prices on application, with full description of sizes.

\section{Bulbs.}

Our success the past year in handling bulbs has been unprecedented. Everywhere we hear of the great success and satisfaction given of 'the quality and character of flowers produced from the bulbs supplied our customers the past season, and we are better prepared the coming year to give our friends the very finest quality of goods procurable, and at the lowest possible prices, and at the earliest possible moment. Do not place your order until you get our figures.

- Catalogues will be mailed as soon as possible, containing list of varieties and prices.

\section{Remember}

This stock is especially grown for us by the very best growers, and should not be compared to that usually offered in the open markets.

\section{Special Offer to Florists for Easter and Store Decorations.}

We are now growing a large quantity of different varieties of 1llooming Plants especially for retailers. They are all finely furnished and well set with flowers, and we ask all florists whose trade demands such plants to write to us.

\section{Azaleas.}

We have an immense stock of these popular and showy blooming plants, embracing ${ }^{-}$the verv cream of thi New York market varieties, in all sizes, from specimens in five-inch pots up to extra large plants.

\section{Genistas.}

We have one entire house, $200 \times 25$ feet, filled entirely with this elegant decorative plant embracing the twelve best sorts grown, all grown with plenty of room, and are elegant plants in six and seven inch pots; also specimens in ten and twelve inch pots. 


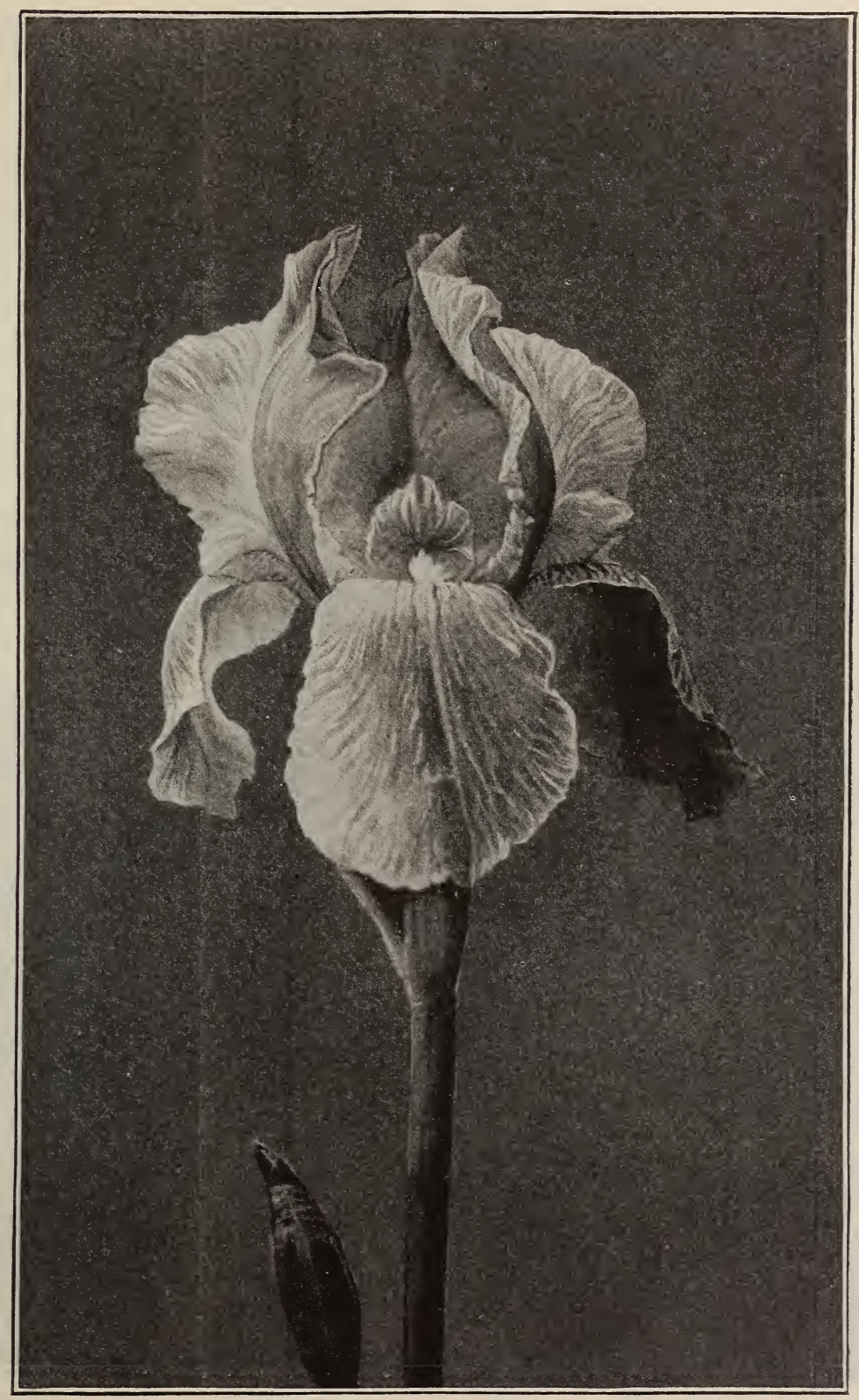

IrIS Germanic..

See Page 62. 


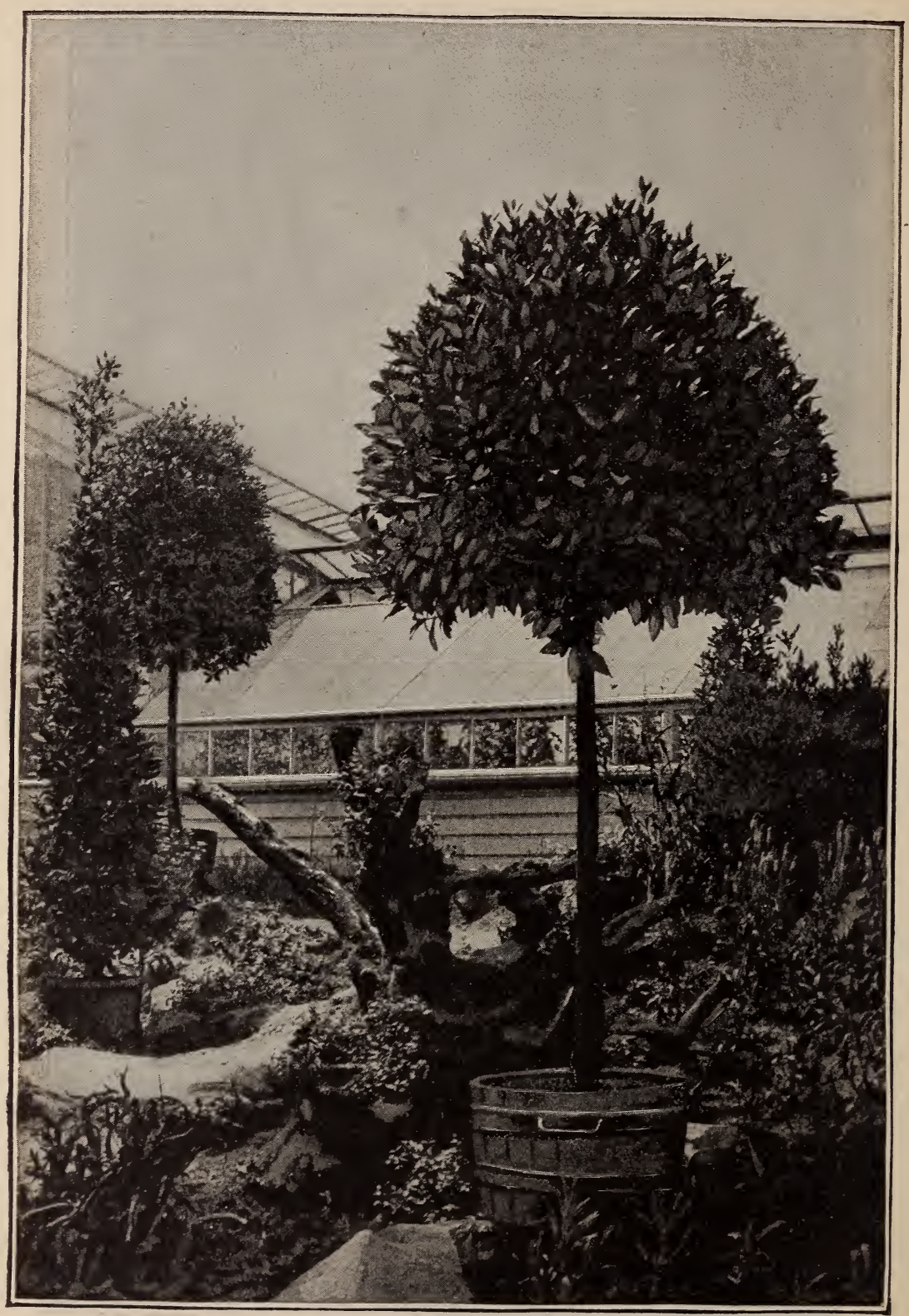

Laurus Nobilis. (Sweet Bay.)

See Paze 70. 


\section{Hydrangea Otaksa.}

A fine-lot of this Easter favorite will be offered, bearing fine large, perfect heads, which we can sell in assorted sizes.

\section{Rhododendrons.}

An extremely beautiful assortment of finely colored varieties of this popular class of flowering plants, in great demand, where offered. Nothing can be finer than a few finely headed rhododendrons in full bloom interspersed among the commoner sorts of blooming plants at an Easter decoration.

In addition to the above we have a fine stock of miscellaneous plants, suitable for display purposes, which will be found valuable and profitable.

Prices and sizes of the above on application.

\section{Tools, Implements, Fertilizers, Insecticides, Etc.}

Axe-

With handle. Medium size........... \$1 00 "، 6

Heavy................ 125

Pruning.............. 40

Barrow-

Garden. No. 1 Small Size.

375

66

“ 2 Medium.............. 4 \%

" 3 Large .................. 500

\section{Bellows-}

Powder. Large .................... 160

Small .................. 80

Vaporizer. Large. .................. 175

" Small................... 100

Brush, Aphis.................... 50

Chisel, Grafting................... 50

Dibber. Two sizes.................. 40

Floral Tool Set-

Four pieces with long handles ..........100

Forks-

Digging. 4 tines................. 65

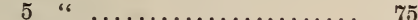

Mauure. 4 tines................... 65

" 5 " 5 .................... 75

Hay. 2 tines ....................... 35

" 3 " $\quad \ldots \ldots \ldots \ldots \ldots \ldots \ldots \ldots, 45$

Hickory Stable ................... 45

Fumigator-

Excelsior. 4 qt. size..................2 200

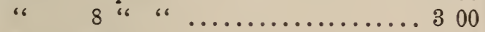

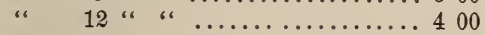

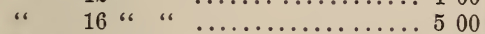

Glasses, Hyacinth-

Tall. Assorted colors.........doz., \$1.50.

Low (Tye's patent). Assorted colors...... .................... per doz., \$1.50.

Gloves, Gardener's.................pair, 75

Glass Cutter and Glazing Tools.per doz., 100

Glazing P sints. 1,000 in a box...per box, 75

Glazing Pincers .
Hoes- Each

Draw or Field. 5 inch ............\$0 25

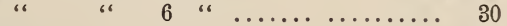

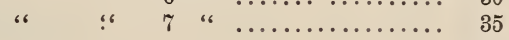

“ 68 " 8 " $8 \ldots \ldots \ldots \ldots \ldots \ldots, 40$

Scuffle or Push. 5 inch............ 50

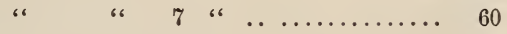

9 “ $9 \ldots \ldots \ldots \ldots \ldots \ldots \ldots \ldots \ldots \ldots \ldots$

Warren. 1st size. Small............ 45

" $2 \mathrm{~d}$ " Medium .......... 55

" $3 \mathrm{~d}$ " Large.............. 65

Onion. 1 prong.................. 25

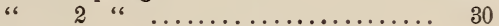

\section{Hooks-}

Bill........................ 115

Brush ........................... 160

Grass, or Sickle. Size No. 1.......... 25

" " " " " $"$ " $6 \ldots \ldots \ldots \ldots .40$

، 6 6 ، $63 \ldots \ldots \ldots \ldots \ldots, 45$

Potato....................... 40

Horse Lawn Boots......... per set of four. .10 00

Hose, Rubber-

Very best, $1 / 2$ inch............. per foot, 14

" " $3 / 4$ " " $\ldots \ldots \ldots \ldots \ldots$ "

" " 1 " $1 \ldots \ldots \ldots \ldots \ldots$. " 20

Good Quality, $1 / 2$ inch......... " 10

". " $3 / 4$ " " $\ldots \ldots \ldots \ldots$ "

، ، 1 ، $1 . \ldots \ldots \ldots \ldots$ ، 15

Hydronette Pump............... 500

Knives-

Asparagus ..................... 100

Grass or edging. With handle......... 49

Budding..............each, 75 cents and 100

Pruning...........each, 75 cents, $\$ 1$ and 125

Labels, Wooden-

Pot or (raraen, 4 inch, pointed

a per $1,000 \ldots \$ 046 \$ 060$

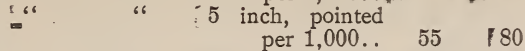

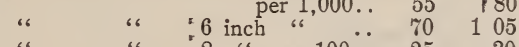

" " 6 " 8 "

50 " “ 12 " 12 " 12 . 
Tools, Implements, etc.-Continued.

Labels, Wooden-

Tree or Plant, notched. $31 / 2$ inch, per 1,000 .. $\$ 035 \$ 055$ copper wired. $3 \frac{1}{2}$ inch..per 1,000 .. $130 \quad 150$ notched. 6 inch,

\section{Lawn Mower-} per 1,000 .. $100 \quad 130$

New Model. 10 inch. Net price......6 625

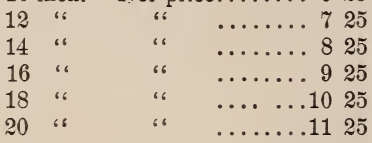

Line-

Garden. Braided Linen........per $100 \mathrm{ft} ., \quad 35$ Cotton ............ " " 20

Mattock. With handle.............. 100 Mats, Archangel............ per doz., $\$ 6.00$.

Mole Trap, Hale's................... 175 Nozzle, Hose-

Brass, with stop cock, spray and stream tips

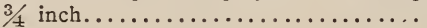

1 inch...................... 90

Graduating Spray. $3 / 4$ inch .......... 75 1 inch..................... 90

\section{Rake-}

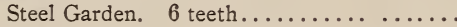

" $6 \quad 8$ " $8 \ldots \ldots \ldots \ldots \ldots \ldots \ldots \ldots, 25$

10 ،

12 " $\ldots \ldots \ldots \ldots \ldots \ldots \ldots .35$

14 " $4 \ldots \ldots \ldots \ldots \ldots \ldots .40$

16 ، $6 \ldots \ldots \ldots \ldots \ldots \ldots . \ldots \ldots \ldots$

Wooden Hay. 12 teeth.............. 25 Lawn. 22 teeth............ 30

Raphia......................... lber., 15 Reels-

Hose. Holding 100 feet of hose....... 2 50 " " 200 " " ....... 30

For Garden Line. Large. . . . . . . . . 750

\section{Rollers, Iron Lawn-}

Small............. 45

\begin{tabular}{|c|c|c|c|c|c|c|}
\hline $\begin{array}{c}\text { Section. } \\
2\end{array}$ & of $\mathrm{Ea}$ & in & $\begin{array}{l}\text { Height. } \\
15 \text { in. }\end{array}$ & $\begin{array}{l}\text { We } \\
125\end{array}$ & & 600 \\
\hline 1 & 20 & " & 20 " & 220 & ") & 1000 \\
\hline 2 & 12 & 6 & $20 "$ " & 300 & "، & 1300 \\
\hline 3 & 12 & " & 20 ، & 450 & ، & 18 \\
\hline 2 & 12 & " & 24 " & 400 & "6 & 15 \\
\hline 2 & 12 & " & 28 “" & 500 & "6 & 18 \\
\hline
\end{tabular}

Saws-

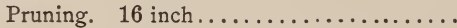

" $\quad 18$ " $6 \ldots \ldots \ldots \ldots \ldots \ldots \ldots \ldots, 60 \ldots \ldots$

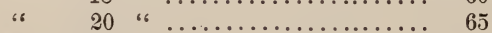

Scissors. Grape Thinning.......... 60 Scythes-

Lawn. 30 to 38 inch............... 110

Hay or Grass. 30 to 38 inch.......... 75

Snath or Handles................. 65

Bush...................... 75

Scythe Stones-

English Round Talacre.............. 10

American.................... 10
Shears- Each.

Pruning. 'Solid Steel. $71 / 2$ inch........ $\$ 100$

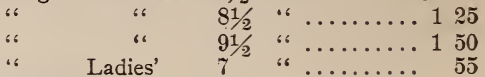

Shovels-

Best Quality. Square point, long or short handle....................... 75

Ames Extra Quality............... 100

Round Point............... 75

Potato. Steel wire. Large........... 140

Spades-

Best quality. Square point, long or short handle....................... 75

Ames' Extra Quality............... 100

Sprinkler-

Lawn. 4 arm .................. 325

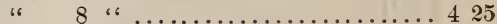

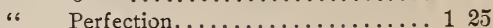

Plant. Rubber bulb. $1 / 2$ pint........ $5 \tilde{5}$

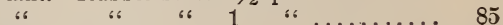

Stakes, Plant- Per 100

Round, tapering, painted green, $2 \mathrm{ft}$. ...\$1 50

\begin{tabular}{|c|c|c|c|c|c|}
\hline " & “ & 66 & 6 & $3 \%$ & 2 \\
\hline "6 & " & $"$ & "6 & $4: 6$ & $\ldots 400$ \\
\hline “6 & “ & $\therefore$ & 6 & 5 “ & .. 475 \\
\hline Square & $" 6$ & 6 & $\because$ & $2 \pi$ & .. 1 \\
\hline 6 & “ & “ & ، & $3 "$ & 2 \\
\hline “ & 6 & “ & $\because$ & $4^{66}$ & . \\
\hline “6 & $\because$ & $" 6$ & $\because$ & 5 & . \\
\hline & 66 & 6 & i6 & $6 \div$ & \\
\hline
\end{tabular}

Dahlia Poles, Round Green. $3 \mathrm{ft}$. ...... 350 $\because 6$ " 6 "6 4 "6 $\ldots \ldots \ldots 475$ " 6 "

Syringe-

Brass. No. A................... 200

" $" 2 \ldots \ldots \ldots \ldots \ldots \ldots \ldots \ldots \ldots 325$

" $11 \ldots \ldots \ldots \ldots \ldots \ldots \ldots . \ldots 400$

Thermometer-

Tin Japanned. 7 inch $\}$

" " 8 " 8 " $3 \ldots \ldots \ldots \ldots, 15$

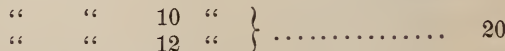

Cylindrical or Dairy............... 60

Registering...................... 2 75

Hot-bed and Mushroom Bed. 12 in...... 200

Tree Pruner-

Water's. $4 \mathrm{ft} . \ldots \ldots \ldots \ldots \ldots \ldots \ldots \ldots 100$

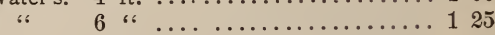

" $\quad 8$ " $\ldots \ldots \ldots \ldots \ldots \ldots \ldots \ldots \ldots 150$

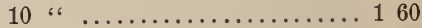

Tree Scraper................... 45

Trowel-

Solid Steel. Welded extra strong. 6־in... 50

$\begin{array}{llllll}6 & 6 & 6 & 8 & 6\end{array}$

American. 5 in................. 10

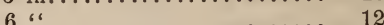

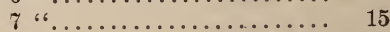

8 " $\ldots \ldots \ldots \ldots \ldots \ldots \ldots \ldots, 18$ 
Tools, Implements, etc.-Continued.

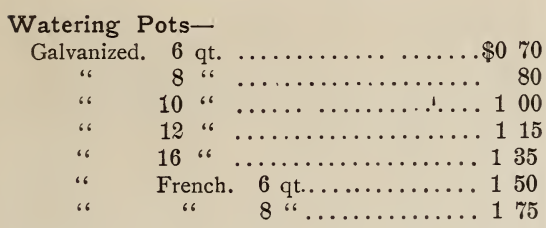

Wax-

Per $\mathrm{lb}$

For Grafting..................\$0 25

French. $1 / 4$ and $1 / 2$ lb. packages ...... 70

Weeders- Each.

Hand Excelsior.................\$0 10

" Noyes'.................... 18

"Hazeltine's................ 15

Insecticides-

Fir Tree Oil................ pt., 75 cts.

Persian Powder.................\$0 50

Paris Green................... 25
Insecticides-continned.

Per 1b.

Powdered White Hellebore...........\$0 30

Slug Shot. ............5 lbs., 25 cts.

Hammond's Grape Dust for Mildew....... $\ldots \ldots \ldots \ldots \ldots \ldots$ lbs., 30 cts.

Flowers of Sulphur............. 06

Carbolic Soap. In $1 / 4$ lb. packages....... 50

Tobacco Soap................... 35

Gishurst's Compound............... 40

Whale Oil Soap............100 lbs., $\$ 7 . \quad 15$

Tobacco Stems............50 lb. bale, 80

Tobacco Dust. Very finely sifted........ 05

\section{Fertilizers-}

Per ton of

Lawn.................\$3 $00 \quad \$ 4500$

Bone Meal............................ 30000

Cotton Seed Meal...........2 $50 \quad 3500$

Peruvian Guano............ $250 \quad 4000$

Ground Bone..............250 4000

Crushed Bone............. $250 \quad 4500$

Unleached Canadian Ashes.....2 $200 \quad 2000$

\section{Books.}

A list of Horticultural and Gardening books, a selection of which should be in the library of every florist, gardener, or amateur grower, and lover of plants and flowers.

The Illustrated Dictionary of Gardening. A practical Encyclopedia of Horticulture for Gardeners and Botanists. By G. Nicholson, and others. Illustrated with numerous full-page plates printed in colors, and over 2,000 accurately executed rood cuts in the text. The most complete work of the kind ever published, giving full particulars regarding all kinds of exotic and hardy plants and vegetables in cultivation. 4 vols., imp. 8 vo., including the supplement of new species and varieties, cloth, gilt edges, \$20.

Henderson's New Handbook of Plants and General Horticulture. A dictionary of the principal cultivated plants, with short descriptions and an extensive Glossary of Botanical and Horticultural terms. 1 vol., imp. 8vo, cloth, $\$ 4$.

The Orchid Grower's Manual. By Benjamin Samuel Williams. New edition, enlarged and revised, containing descriptions of upwards of 1,470 species and varieties. Illustrated with a large number of full page and double page engravings, and some small engravings in the text. 8vo, cloth, $\$ 7$.

Choice Stove and Greenhouse Flowering and Ornamental-Leaved Plants. By B. S. Williams. With descriptions of upwards of 1,100 species and varieties with instructions for their culture. With colored frontispiece and many engravings on wood. 2 vols., $12 \mathrm{mo}$, cloth, $\$ 5$.
Parsons on the Rose. A treatise on the Propagation, Culture and History of the Rose. By Samuel B. Parsons. New and revised edition, with numerous illustrations. $12 \mathrm{mo}$, cloth, $\$ 1$.

Practical Floriculture, a Guide to the Successful Propagation and Cultivation of Florists' Plants. By Peter Henderson. New and enlarged edition. With numerous illustrations. $12 \mathrm{mo}$, cloth, $\$ 1.50$.

The Rose. By B. Ellwanger. A Treatise on the History, etc., of the Rose, with directions for its Cultivation, including a descriptive alphabetical list of 956 varieties. $12 \mathrm{mo}$, cloth, $\$ 1.25$.

Bulbs. By Edward Sprague Rand. A Treatise on Hardy and Tender Bulbs and Tubers. The best book on the subject for Americans, being written especially for the conditions, climate, etc., of this country. $12 \mathrm{mo}$, cloth, $\$ 2.50$.

The Silva of North America. A description of the trees which grow naturally in North America, exclusive of Mexico. By Charles Sprague Sargeant. Illustrated with figures drazon from nature by Charles Edvoard Faxon. To be complete in 12 volumes, 4 to, each volume containing 50 plates; cloth, $\$ 25$ per volume. Vols. I. and II. now ready.

The Native Flowers and Ferns of the United States, in their Botanical, Horticultural and Popular Aspects. By Thomas Meehan. Illlustrated with very many chromo-lithographs. Two series, together 4 vols., 8vo., cloth, \$28. 


\section{Books-Continued.}

Practical Camellia Culture. A Treatise on the Culture and Propagation of Camellia Faponica. By Robert J. Halliday. Illustrated with five colored plates and fifty wood engravings. $12 \mathrm{mo}$, cloth, $\$ 2$.

Practical Azalea Culture. A Treatise on the Propagation and Cultivation of Azalea Indica. By Robert J. Halliday. With illustrations. $12 \mathrm{mo}$, cloth, \$2.

The Tuberous Begonia, Its History and Cultivation With a select list of varieties. $12 \mathrm{mo}$, paper, 50 cents.

Chrysanthemum Culture for America. By Jas. Morton. Full instructions for growing flowers for exhibition purposes, etc. $12 \mathrm{mo}$, cloth, $\$ 1$.

Gardening for Pleasure. A guide to the Amateur in the Fruit, Vegetable and Flower Garden, with full Directions for the Greenhouse and Window Garden. By Peter Henderson. With illustrations. $12 \mathrm{mo}$, cloth, \$2.

Gardening for Profit. A Guide to the Successful Culture of the Market and Family Garden. With numerous illustrations. By Peter Henderson. New edition, entirely rewritten and greatly enlarged. $12 \mathrm{mo}$, cloth, $\$ 2$.

How the Farm Pays. By William Crozier and Peter Henderson. Full information in a popular, not scientific, style. Profusely illustrated. 3vo, cloth, $\$ 2.50$.

New American Farm Book. By R. L. Allen, and revised and enlarged by Lewis F. Allen, Treating on all the various branches of industry connected with the farm. $12 \mathrm{mo}$, cloth, $\$ 2.50$.

Grasses of North America, for Farmers and Students. By Prof. W. J. Beal. The most recent and complete work on American Grasses; written in a popular style which can be comprehended by those who have not studied botany. With many illustrations. 8vo, cloth, \$2.50.

Grasses and Forage Plants. A Practical Treatise on the Fodder Plants of the United States and the British Provinces. By Charles L. Flint. New revised edition. With numerous illustrations. $12 \mathrm{mo}$, cloth, \$2.

Success with Small Fruits. By E. P. Roe. $12 \mathrm{mo}$, cloth, with numerous illustrations, $\$ 1.50$.

Play and Profit in My Garden. By E. P. Roe. $12 \mathrm{mo}$, cloth, $\$ 1.50$.
The Home Acre. By E. P. Roe. A popular and instructive book written in an entertaining style, by the well-known and popular novelist, whose country seat at Cornwall-on-the-Hudson was one of the most beautifully laid out places in the country at the time of the author's death. $12 \mathrm{mo}$, cloth, $\$ 1.50$.

How Crops Grow. A Treatise on the Chemical Composition, Structure and Life of the Plant. By Prof. Samuel W. Johnson, of Yale College. New edition, entirely rewritten and greatly enlarged. With numerous illustrations. $12 \mathrm{mo}$, cloth, \$2.

How Crops Feed. By Prof. Samuel WV. Johnson. A Treatise on the Atmosphere and the Soil as relating to the Nutrition of Agricultural Plants. With illustrations. $12 \mathrm{mo}$, cloth, $\$ 2$.

Elements of Agriculture. A Book for Young Farmers. By George E. Waring, Jr. New edition, carefully revised. $12 \mathrm{mo}$, cioth, $\$ 1$.

The Propagation of Plants. Describing the processes of Hybridizing and Crossing Species and Varieties, and also of many different modes by which the cultivated plants may be multiplied. By Andrew S. Fuller. With illustrations. $12 \mathrm{mo}$, cloth, $\$ 1.50$.

Practical Forestry. A Treatise on the Propagation, Planting and Cultivation, with descriptions of the Indigenous Trees of the United States, with notes on a large number of valuable exotic species. By Andrew S. Fuller. Illustrated. $12 \mathrm{mo}$, cloth, $\$ 1.50$.

The Nursery Book. A Complete Handbook of Propagation and Pollination. By Prof. L. H. Bailey, of the Cornell Experiment Station. The book tells plainly and briefly what every one who sows a seed, makes a cutting or sets a graft wants to know. With about 100 illustrations. $12 \mathrm{mo}$, cloth, $\$ 1$.

The Horticulturist's Rule Book. By L. H. Bailey. Contains a great many rules and receipts used by florists, gardeners, farmers, etc., etc. Revised edition. 12mo, cloth, \$1.

Ornamental Gardening for Americans. A Treatise for beautifying Homes, Rural Districts and Cemeteries. By Elias A. Long. With nu. merous illustrations. $12 \mathrm{mo}$, cloth, $\$ 2$.

Truck Farming at the South. A Guide for the Raising of Vegetables for Northern Markets. By Dr. A Oemler. Gives full cultural directions; also methods for packing. With illustrations. $12 \mathrm{mo}$, cloth, $\$ 1.50$.

[त्डs Any of the above books will be sent by mail or express prepaid on receipt of the stated price. Any other Horticultural or Farm books can be supplied, and at the publisher's price ; and subscriptions for any Horticultural paper or magazine will be received at regular rates. 


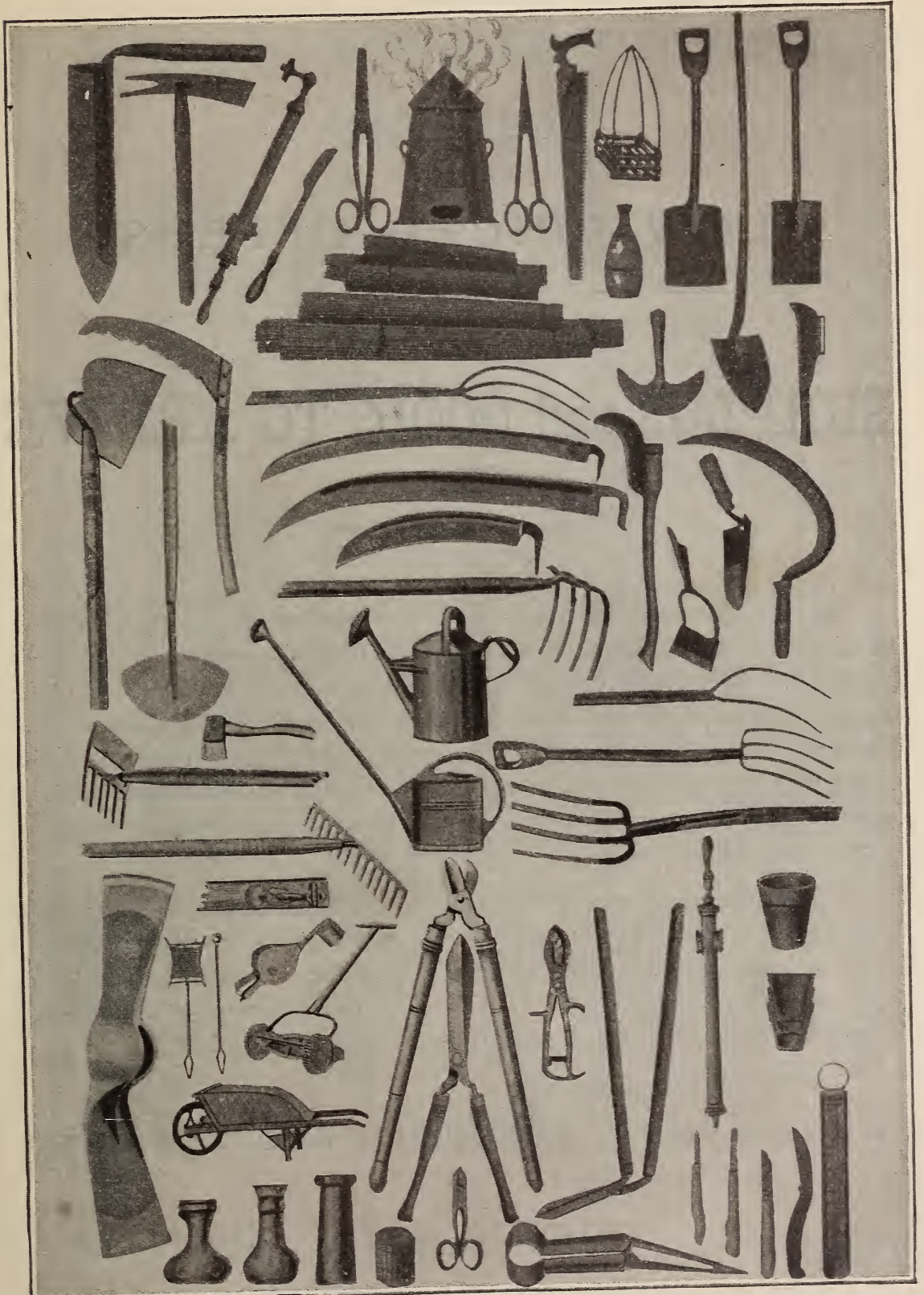

Useful Gardenixg Implements. 


\section{$\underline{S P E C I A L}$}

\section{LOW-PRICED OFFERS}

\section{- $\mathrm{OF}-$ \\ SURPLUS and GOODS TO ARRIVE}

We are constantly receiving very large shipments of the many kinds of goods offered in this list, and issue at different periods of the year, special low-priced offers, both of "Goods to Arrive" and Surplus Stock. Prices therein quoted are always very low and are taken advantage of by all shrewd men in our business. By writing us for these quotations, which apply to Florists', Gardeners' and Jobbers' wants, an immediate reply is guaranteed, and your name will be placed upon our special circular list, for the articles you name.

Should the price on any article quoted in this list not be exactly-in keeping with those of any other house, and you wish to favor us with a share of your business, write us to this effect, and we will mail you our special offer in quantity, which we have now ready on Flower Seeds, Vegetable Seeds, Orchids, Bulbs, Hardy Plants, \&c.

Remember that we are now

NURSERYMEN, SEEDSMEN AND FLORISTS.

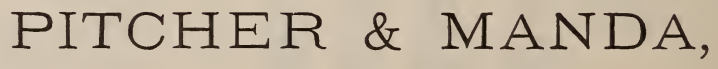

SHORT HILLS, 


\section{THE CATALOGUES IVE PUBLISH.}

Besides publishing our general catalogue, representing under one cover the many departments of our establishment, we issue separately, and have now ready for mailing, catalogues pertaining to the different departments, as follows:

\section{Our Grand Annual. \\ Orchids and Cypripediums. \\ Stove and Greenhouse. \\ Hardy Perennials. \\ New and Rare Bulbs. \\ Seeds.}

These catalogues will be found to contain a more extended list of all varieties worthy of cultivation, and, when desired, we will send any or all upon application.

SEE OPPOSITE PAGE FOR SPECIAL OFFERS. 


\section{IF YOU \\ KEEP A \\ STORE}

\section{HOME COLLECTIONS}

OF

FLOWER AND VEGETABLE

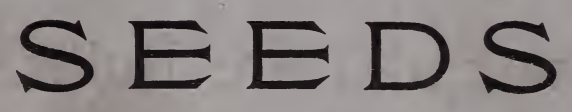

The most salable and profitable thing now in the market. Anyone can sell them and realize 150 per cent. profit. 